University of Tennessee Health Science Center

UTHSC Digital Commons

\title{
Predictors and Mediators of Long-term Functional Limitations in the Older Adult Population
}

Teresa Maria Bell

University of Tennessee Health Science Center

Follow this and additional works at: https://dc.uthsc.edu/dissertations

Part of the Health and Medical Administration Commons

\section{Recommended Citation}

Bell, Teresa Maria , "Predictors and Mediators of Long-term Functional Limitations in the Older Adult Population" (2014). Theses and Dissertations (ETD). Paper 332. http://dx.doi.org/10.21007/ etd.cghs.2014.0025.

This Dissertation is brought to you for free and open access by the College of Graduate Health Sciences at UTHSC Digital Commons. It has been accepted for inclusion in Theses and Dissertations (ETD) by an authorized administrator of UTHSC Digital Commons. For more information, please contact jwelch30@uthsc.edu. 


\title{
Predictors and Mediators of Long-term Functional Limitations in the Older Adult Population
}

\begin{abstract}
With nearly 40 million Americans being treated for nonfatal injuries in emergency departments annually, the burden of nonfatal injury in the United States is extensive. The longterm functional consequences of these injuries can be enduring and far-reaching, especially for older adults. Although studies have reported that persistent functional deficits exist after injury, less information is known about long-term recovery patterns and the factors that influence functional outcomes.

The primary aims of this study are to (1) classify differences in long-term, longitudinal changes in functional limitations within the injured population, (2) identify individual characteristics that predict recovery after injury and (3) assess whether medical care use mediates the relationship between longterm changes in functional limitations and significant predictors of outcomes after injury, specifically insurance status. Longitudinal survey data from the Health and Retirement Study (HRS) was analyzed using group-based trajectory modeling and mediation analysis.
\end{abstract}

The trajectory analysis identified five distinct functional trajectories with the following characteristics: Trajectory 1 - consistently low functional limitations scores (18.9\%), Trajectory 2 - increase in functional limitations after injury followed by a gradual, but not complete recovery (46.3\%), Trajectory 3 - increase in functional limitations followed by further decline in functioning over time (10.5\%), Trajectory 4- increase in functional limitations after injury followed by a gradual, complete recovery (13.4\%), and Trajectory 5consistently high functional limitations scores (10.8\%). Regression analyses showed that women, individuals with multiple health conditions, and individuals with no insurance and public insurance were more likely to belong to trajectories with poorer functional outcomes.

The mediation analysis found that public insurance was associated with increased functional limitations relative to private insurance. The total effect of public insurance on functional limitations was partially mediated by medical care use. Doctor visits was the only significant medical care use mediator for individuals with public insurance. The relative total and direct effects of being uninsured on functional limitations were not significant. However, the indirect effect of being uninsured on functional limitations was significant, indicating that medical care use may suppress the effect of being uninsured on functional outcomes. Prescription drug use was the only significant mediator of the effect of not having insurance on functional status.

These results illustrate that distinct courses of recovery after injury in the older adult population exist. Furthermore, personal characteristics of individuals can be used to predict functional trajectories. This study also demonstrated that insurance status is a significant predictor of both functional outcomes and medical care use after injury. Insurance status was found to exert its effect on health outcomes both directly and indirectly through medical care. Ultimately, the findings from this study can be used to improve the understanding of how individuals' functional outcomes differ after injury and the causal processes that determine these outcomes. This knowledge may lead to tailored policies and treatments that improve quality of life after injury.

\section{Document Type}

Dissertation

\section{Degree Name}

Doctor of Philosophy (PhD) 


\section{Program}

Health Outcomes and Policy Research

Research Advisor

Ben Zarzaur, M.D., M.P.H.

\section{Keywords}

aging, functional status, injury, mediation, trajectory

\section{Subject Categories}

Health and Medical Administration | Medicine and Health Sciences

\section{Comments}

Two year embargo expired May 2016 


\title{
Predictors and Mediators of Long-term Functional Limitations after Injury in the Older Adult Population
}

\author{
A Dissertation \\ Presented for \\ The Graduate Studies Council \\ The University of Tennessee \\ Health Science Center
}

\author{
In Partial Fulfillment \\ Of the Requirements for the Degree \\ Doctor of Philosophy \\ From The University of Tennessee
}

By

Teresa Maria Bell

May 2014 
Copyright $@ 2014$ by Teresa Bell. All rights reserved. 


\section{ACKNOWLEDGEMENTS}

I gratefully acknowledge my family for their love and support. To my mother, Linda Bell, who read to me and nurtured me. To my father, Edward Bell, who started teaching me algebra before I could pronounce it. To my grandfather, John Kress, who showed me the importance of finding work that inspires you. To my grandmother, June Kress, who gave me a deep appreciation for art and nature. I am forever grateful.

I am thankful for my friends who shared this graduate school journey with me, particularly Courtnee Melton, Mustafa Hussein, Dee Helton, Angela Arnold, Crystal Baker, and Matt Lovett. Thank you for your guidance and advice, your humor, taking the time to listen, and for reminding me of all those deadlines I would have forgotten. I am especially thankful to Jeff Morrison for his love and generosity. I am also grateful for my cats, Rupert and Gatsby, who kept me company during many late nights. Without their support, this process would have been longer, harder, and much lonelier.

To my academic mentors and committee members, I am incredibly appreciative of the time, energy, and guidance you provided to me. I would like to thank my research advisor, Dr. Ben Zarzaur, for his willingness to serve as my mentor throughout my $\mathrm{PhD}$ journey and for all the opportunities he has provided me. I am forever indebted to George Relyea for his help, advice, and encouragement - this dissertation would not have been possible without him. I am also thankful for all the wonderful advice, feedback, and friendship I received from committee members Esra Ozdenerol, Robert Nolly, and Junling Wang. I would also like to thank Dr. Don Thomason and all of the College of Graduate Health Sciences staff for all of their help and support throughout my studies.

Lastly, I would like to thank Prince, Nina Simone, Glenn Gould, Fiona Apple, Lykke Li, David Bowie, and Daft Punk for providing the soundtrack to my life while working on this dissertation. 


\begin{abstract}
With nearly 40 million Americans being treated for nonfatal injuries in emergency departments annually, the burden of nonfatal injury in the United States is extensive. The long-term functional consequences of these injuries can be enduring and far-reaching, especially for older adults. Although studies have reported that persistent functional deficits exist after injury, less information is known about long-term recovery patterns and the factors that influence functional outcomes.

The primary aims of this study are to (1) classify differences in long-term, longitudinal changes in functional limitations within the injured population, (2) identify individual characteristics that predict recovery after injury and (3) assess whether medical care use mediates the relationship between long-term changes in functional limitations and significant predictors of outcomes after injury, specifically insurance status. Longitudinal survey data from the Health and Retirement Study (HRS) was analyzed using group-based trajectory modeling and mediation analysis.
\end{abstract}

The trajectory analysis identified five distinct functional trajectories with the following characteristics: Trajectory 1 - consistently low functional limitations scores $(18.9 \%)$, Trajectory 2 - increase in functional limitations after injury followed by a gradual, but not complete recovery $(46.3 \%)$, Trajectory 3 - increase in functional limitations followed by further decline in functioning over time $(10.5 \%)$, Trajectory 4 increase in functional limitations after injury followed by a gradual, complete recovery (13.4\%), and Trajectory 5- consistently high functional limitations scores (10.8\%). Regression analyses showed that women, individuals with multiple health conditions, and individuals with no insurance and public insurance were more likely to belong to trajectories with poorer functional outcomes.

The mediation analysis found that public insurance was associated with increased functional limitations relative to private insurance. The total effect of public insurance on functional limitations was partially mediated by medical care use. Doctor visits was the only significant medical care use mediator for individuals with public insurance. The relative total and direct effects of being uninsured on functional limitations were not significant. However, the indirect effect of being uninsured on functional limitations was significant, indicating that medical care use may suppress the effect of being uninsured on functional outcomes. Prescription drug use was the only significant mediator of the effect of not having insurance on functional status.

These results illustrate that distinct courses of recovery after injury in the older adult population exist. Furthermore, personal characteristics of individuals can be used to predict functional trajectories. This study also demonstrated that insurance status is a significant predictor of both functional outcomes and medical care use after injury. Insurance status was found to exert its effect on health outcomes both directly and indirectly through medical care. Ultimately, the findings from this study can be used to improve the understanding of how individuals' functional outcomes differ after injury 
and the causal processes that determine these outcomes. This knowledge may lead to tailored policies and treatments that improve quality of life after injury. 


\section{TABLE OF CONTENTS}

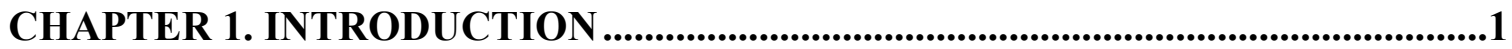

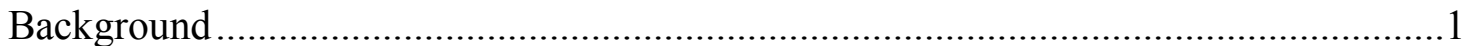

Purpose of the Study ..........................................................................................

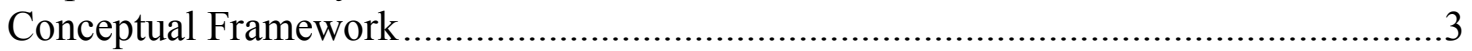

Models of Health Trajectory as a Health Outcome.................................................

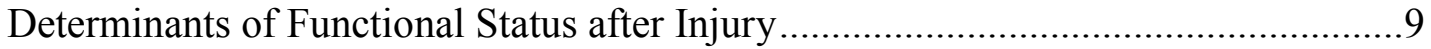

Conceptual Model for the Proposed Study ......................................................... 11

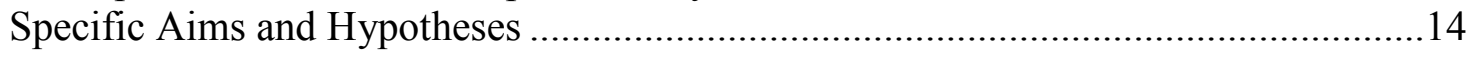

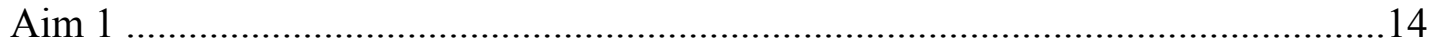

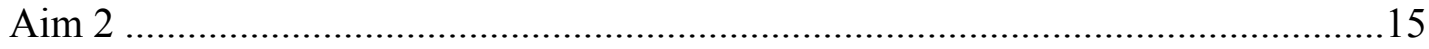

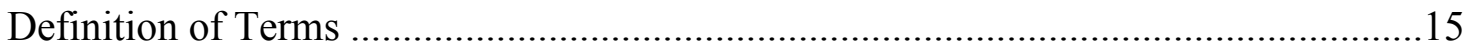

CHAPTER 2. REVIEW OF THE LITERATURE .....................................................17

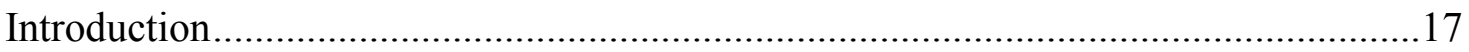

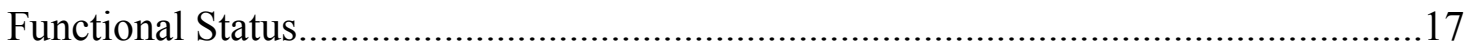

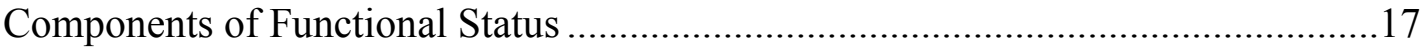

The Relationship between Functioning and Disability .......................................18

Functional Status Indicators and Associated Functional Domains ...........................20

Long-term Functional Outcomes after Injury in Adults (2+ Years Post-injury) .......22

Patient Characteristics that Predict Functional Status after Injury ..........................24

Healthcare-related Predictors of Functional Status after Injury .............................27

Effects of Insurance Status on Medical Care Use after Injury................................28

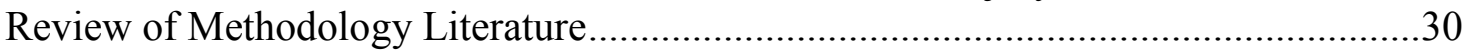

Group-based Trajectory Modeling in Injury Research ....................................... 31

Mediation Analysis in Health Services Research ....................................................31

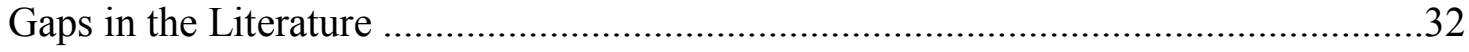

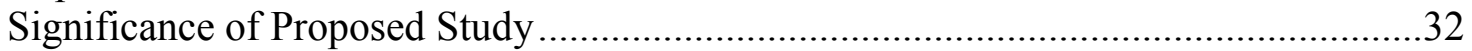

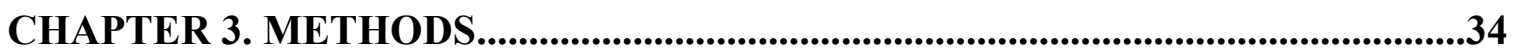

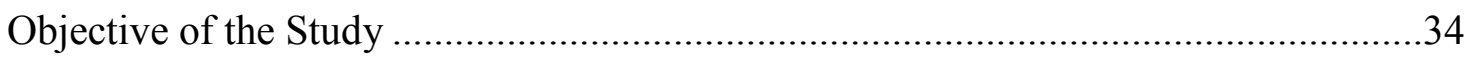

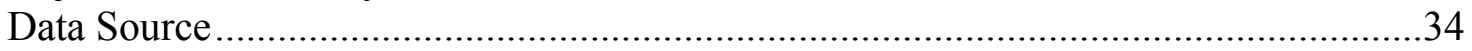

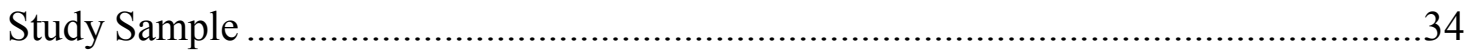

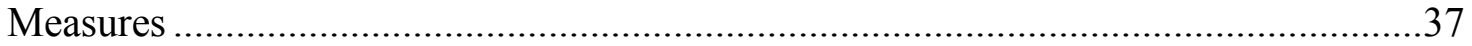

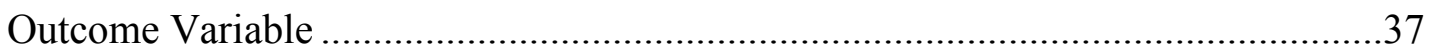

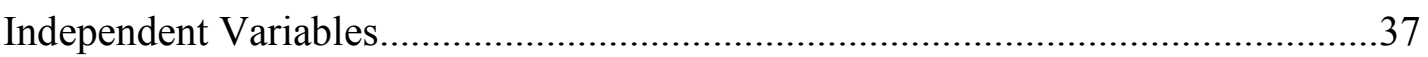

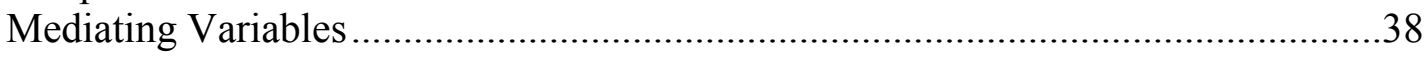

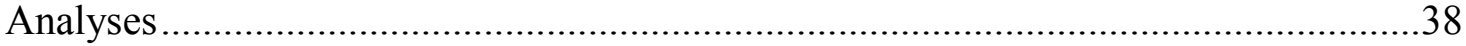

Part I. Classification of Functional Status Trajectory after Injury and

Identification of Predictors of Long-term Functional Outcomes.............................40

Part II. Assessing the Role of the Relationship between Health Insurance and Medical Care Use on Long-term Functional Outcomes 


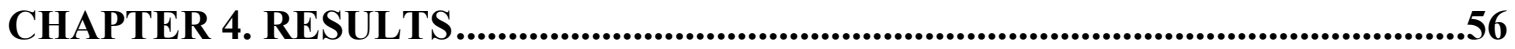

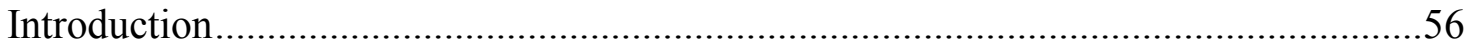

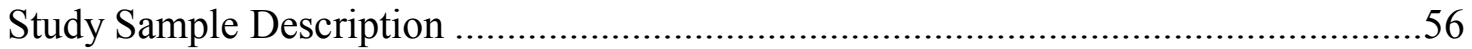

Comparison of Injured Cohort to Total HRS Sample.............................................56

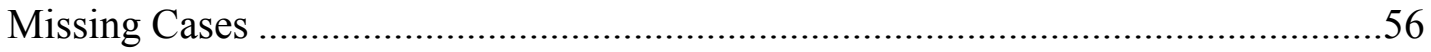

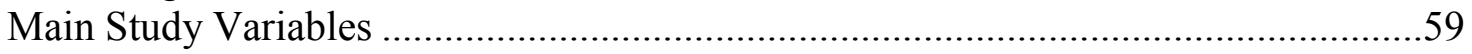

Functional Limitations Scale Creation..................................................................59

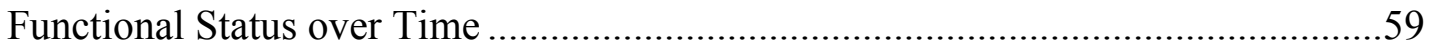

Independent Variables over Time ……………………......................................59

Mediating Variables over Time ……………………….....................................63

Correlations among Study Variables ......................................................................63

Part I. Classification of Functional Status Trajectory after Injury and

Identification of Predictors of Long-Term Functional Outcomes ...............................66

Determining Predictors of Recovery Subgroups ……………..................................77

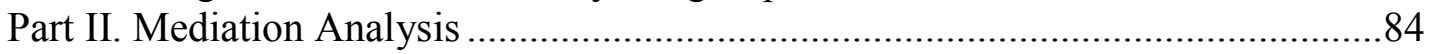

Comparable Coefficient and Standard Error Calculations..........................................101

Relative Total, Direct, and Indirect Effects of Insurance Status...............................101

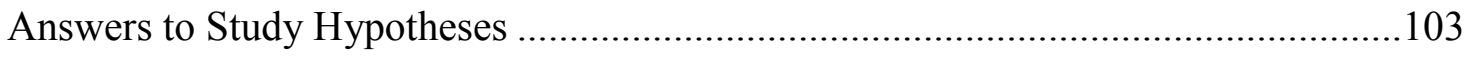

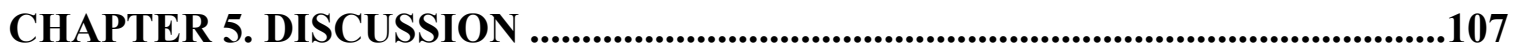

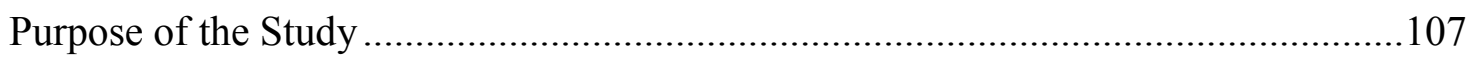

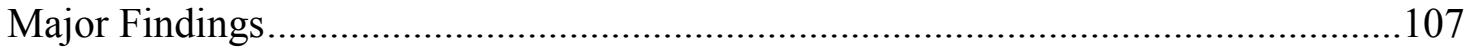

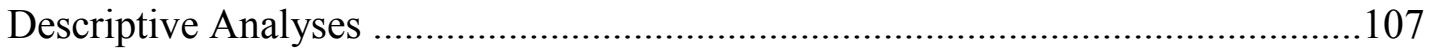

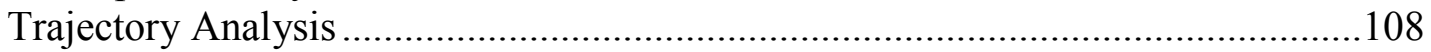

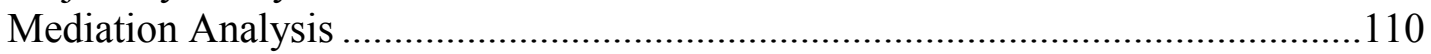

Comparison of Study Findings to Existing Literature ...............................................110

Changes in Long-term Functional Status after Injury.............................................110

Predictors of Long-term Functional Status .............................................................111

Effect of Insurance Status on Medical Care Use after Injury ....................................112

Effect of Insurance Status and Medical Care Use on Functional Outcomes ............112

Research Implications of the Findings...................................................................113

Patient-Centered Outcomes Research ................................................................113

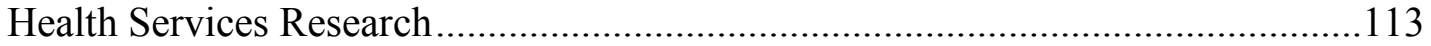

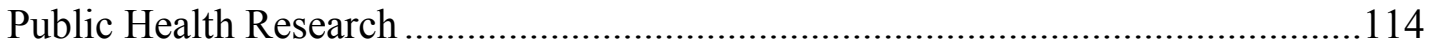

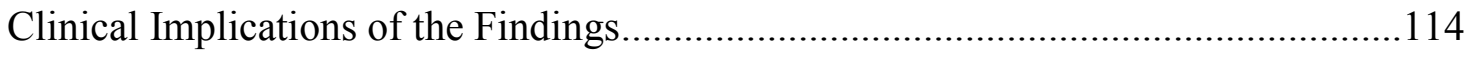

Predictors of Functional Decline ..........................................................................114

Predictors of Functional Improvement ………………….................................115

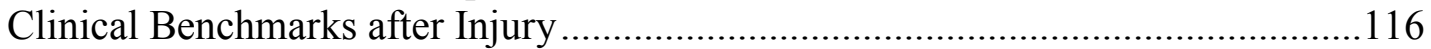

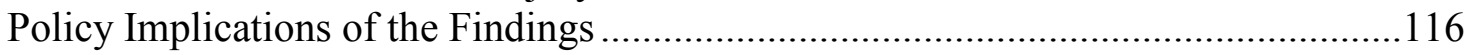

Policies Concerning the Coverage of Medical Services ..........................................116

Policies Expanding Access to Medical Care after Injury for Older Adults under

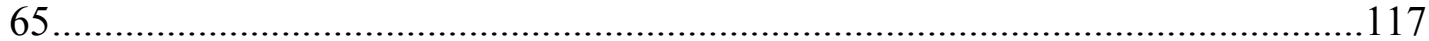

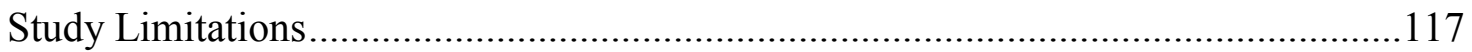

Lack of Direct Information on Respondent Injury Severity ...................................117

Validity of Self-reported Data ............................................................................118

Observation Interval Length ............................................................................118 


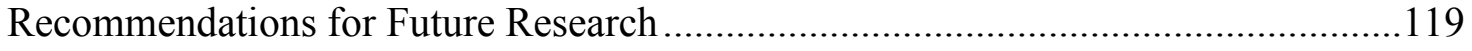

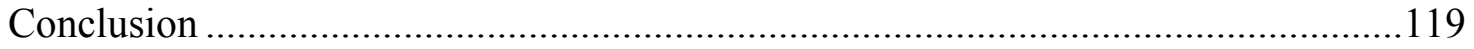

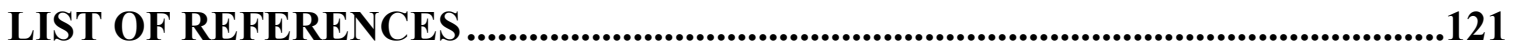

VITA 


\section{LIST OF TABLES}

Table 3-1. 2000 HRS Core Survey Injury Variables......................................................35

Table 3-2. List of Variables for Statistical Models ..........................................................39

Table 3-3. Equations for Rescaled Outcome Variables ...................................................50

Table 4-1. Descriptive Characteristics at Baseline.......................................................57

Table 4-2. Number and Percentage of Cases Missing across Survey Waves .................58

Table 4-3. Functional Limitations Scale Factor Analysis .................................................60

Table 4-4. Reliability Analysis for Functional Limitations Scale Items .........................60

Table 4-5. Functional Outcomes across Survey Waves ..................................................61

Table 4-6. Insurance Status across Survey Waves .........................................................62

Table 4-7. Mediating Variables across Survey Waves.......................................................64

Table 4-8. Correlation among All Study Variables..........................................................65

Table 4-9. BIC Values for Trajectory Models ……………………………………......67

Table 4-10. Bayes Factors for Trajectory Models ............................................................67

Table 4-11. Jeffrey's Scale of Evidence for Bayes Factors ……………………………....67

Table 4-12. Mean and Predicted Functional Limitations Scores .....................................70

Table 4-13. Number and Percentage of Respondents Belonging to Each Trajectory Subgroup .............................................................................................

Table 4-14. Baseline Characteristics for Trajectory Subgroup ........................................75

Table 4-15. Insurance Status and Medical Care Use over Time .......................................78

Table 4-16. Bivariate Logistic Regression Analyses to Distinguish between Trajectory Subgroups

Table 4-17. Multinomial Logistic Regression Analysis of Characteristics that Best Discriminate Functional Status Trajectories 1, 3, 4, and 5 Compared to 2..85

Table 4-18. Model 1 Effect Estimates (Unstandardized) ..................................................91

Table 4-19. Model 2 Effect Estimates (Unstandardized) .................................................93 
Table 4-20. Model 3 Effect Estimates (Unstandardized) .........................................95

Table 4-21. Model 4 Effect Estimates (Unstandardized) ..........................................97

Table 4-22. Model 5 Effect Estimates (Unstandardized) ...........................................99

Table 4-23. Comparable Coefficient Estimates and Standard Errors ...........................102

Table 4-24. Relative Total, Direct, and Indirect Effects ........................................... 104 


\section{LIST OF FIGURES}

Figure 1-1. Minnesota Center for Health Trajectory Research Framework .......................4

Figure 1-2. Iwashyna's Big Hit Trajectory of Recovery after Acute Illness .....................6

Figure 1-3. Iwashyna's Slow Burn Trajectory of Recovery after Acute Illness ...............7

Figure 1-4. Iwashyna's Relapsing Recurrences Trajectory of Recovery after Acute

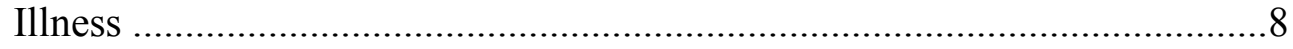

Figure 1-5. Wilson and Cleary's Patient Outcomes Model ............................................10

Figure 1-6. Hadley's Framework..............................................................................12

Figure 1-7. Conceptual Model for the Study ............................................................13

Figure 2-1. International Classification of Functioning, Disability, and Health .............19

Figure 3-1. Simple Mediational Model .....................................................................4

Figure 3-2. Path Diagram for the Mediation Model .....................................................46

Figure 3-3. Equations for Calculating Standard Deviations for $\mathrm{Y}^{\prime}, \mathrm{Y}^{\prime}, \mathrm{M}_{1}, \mathrm{M}_{2}$ ',

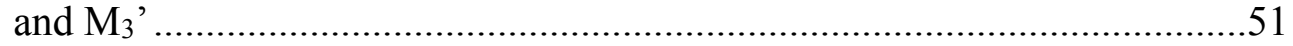

Figure 3-4. Formulas for Comparable Coefficients........................................................53

Figure 3-5. Formulas for Comparable Standard Errors ..................................................54

Figure 4-1. Mean and Predicted Functional Limitations Scores across Survey Waves ..68

Figure 4-2. Predicted Functional Limitations Scores and 95\% Confidence Intervals across Survey Waves.................................................................................69

Figure 4-3. Trajectory Mean Functional Limitations Scores across Survey Waves .......73

Figure 4-4. Mediation Model Effect Estimates …………………………………......105 


\section{CHAPTER 1. INTRODUCTION}

\section{Background}

Among Americans between the ages of 1 and 44, injury leads all other causes of death. The U.S. National Center for Injury Prevention and Control reported that over 175,000 Americans died due to injury in 2009, resulting in 3.49 million years of potential life lost (Centers for Disease Control [CDC], 2012). In one year, the total cost of injury to the U.S. exceeded $\$ 400$ billion, which included over $\$ 80$ billion in direct medical costs and $\$ 326$ billion due to lost productivity (Corso, Finkelstein, Miller, Fiebelkorn , \& Zaloshnja, 2006). Although society pays a high cost for fatal injury in indirect costs associated with lost productivity, nonfatal injuries make up over $99 \%$ of total injuries and account for the majority of direct costs. In 2012, nearly 38 million nonfatal injuries required medical treatment and approximately 2 million of these injuries required hospitalization (Adams, Kirzinger, \& Martinez, 2013). Injury is the sixth most common reason for hospital stay and the second most expensive condition to treat in the hospital setting (Health Cost Utilization Project [HCUP], 2007). The amount of medical spending on nonfatal injuries is nearly 95 times that of fatal injury (Stevens, Corso, Finkelstein, \& Miller, 2006). Compared to fatal injury, nonfatal injury contributes to the majority of the U.S. burden of injury and is also the primary driver of direct costs associated with injury.

Often nonfatal injuries are primarily associated with causing substantial shortterm functional impairments as opposed to lasting effects on health. Many studies examining the burden of injury assume that accidents have only limited direct implications on an individual's future health prognosis (McClellan, 1998). However, the long-term effects and consequences of injury can be enduring and far-reaching. Lasting functional deficits in the injured population can cross multiple domains of health including physical functioning, social functioning, mental health, cognition, and vitality (Inaba, Goecke, Sharkey, \& Brenneman, 2003; Soberg, Bautz-Holter, Roise, \& Finset, 2007; Timmers, Verhofstad, Moons, van Beeck, \& Leenen, 2011). Both severe injury and minor injury have been shown to contribute to the development of lasting secondary conditions such as osteoarthritis, post-traumatic stress disorder, and chronic pain (Saxon, Finc, \& Bass, 1999; Mayou \& Bryant, 2002; Rivar et al., 2008). Additionally, the pain and physical limitations that often follow injury can increase the likelihood of adopting negative health behaviors including excessive alcohol and tobacco consumption and decreased participation in physical activities. Changes in behavioral health can in turn lead to the development of chronic health conditions such as cardiovascular disease, obesity, and cancer (Ponsford, Whelan-Goodinson, \& Bahar-Fuchs, 2007). As a result, many individuals experience a life-long decrease in their quality of life following injury and are at increased risk for disability and early mortality (Mayou \& Bryant, 2002; Evans et al., 2003; Hindmarsh, Hayen, Finch, \& Close, 2009).

Previous research has found that certain populations are more likely to experience poor outcomes following injury than others. Age, race, gender, and income are associated with poor functional outcomes and reduced quality of life following injury, even after 
controlling for injury severity and pre-injury health status (Langley, Derrett, Davie, Ameratunga, \& Wyeth, 2011; Hakmeh, Barker, Spunar, Fox, \& Irvin, 2010;

Staudenmayer, Diaz-Arrastia, de Oliveira, Gentilello, \& Shafi, 2007; Heffernan et al., 2011). Health insurance coverage has also been well documented as a substantial contributor to both functional and long-term mortality outcomes after injury (Hadley, 2007; Singer et al., 2013). Those without insurance are at increased risk for delayed recovery, residual functional deficits, disability, lower quality of life, and mortality (Hakmeh et al., 2010; Rosen, Saleh, Lipsitz, Rogers, \& Gawande, 2009). The precise mechanism for how these patient characteristics play a role in outcome after injury is unknown, however treatment delays, receipt of fewer diagnostic services, decreased health literacy, and reduced likelihood of placement in rehabilitation centers have been proposed (Rosen et al., 2009; Shafi al., 2007).

Unlike demographic predictors of health outcomes, medical care use is a modifiable factor that can increase the likelihood of patients attaining an optimal outcome following injury. Utilization of health services could potentially explain the relationship between individual risk factors and poor health outcomes. The relationship between access to medical care and both patient characteristics and health outcomes has been described in the literature. Patients with no insurance are less likely to obtain any medical care for their injury, more likely not to receive follow-up care, have fewer outpatient and office-based visits, take fewer prescriptions, and are less likely to be placed in rehabilitation than insured patients (Hadley, 2007; Shafi et al., 2007, Heffernan et al., 2011; Claridge et al., 2006). Difficulty accessing health services is associated with adverse health outcomes in many patient populations including the injured (Langley et al., 2011). Investigation into whether and to what extent medical care use mediates health outcomes after injury should be examined.

\section{Purpose of the Study}

The purpose of this study was to investigate how injury impacts long-term health in order to better characterize the recovery process and determine which patients are most at risk for not reaching full recovery. A second objective of this study was to assess the influence of insurance status and medical care use on long-term health outcomes. A better understanding of this relationship could aid in the development of policies that address outcome disparities by modifying insurance coverage and access to health services associated with improved outcomes. This research seeks to support outcomes-based policy planning by elucidating pathways for healthcare-driven improvement in patient populations most vulnerable to long-term deficits in functional ability after injury.

Long-term functional outcomes were evaluated using a person-centered, health trajectory approach. Studying how functional trajectories of distinct subgroups of individuals differ will help characterize patterns of dysfunction and recovery. Additionally, information concerning predictors of functional trajectories can be obtained. This approach provides patient-centered information that can be applied to

developing targeted clinical interventions. A mediation approach to investigating the 
second study objective was used so that findings can potentially be more supportive in guiding policy development. This approach merges both process and outcomes research by identifying which components of health insurance are most effective, the causal process through which they work, and patient characteristics that moderate the relationship between insurance coverage and functional outcomes. Increasing understanding of the processes that lead to better outcomes will facilitate policy development by defining at-risk populations and clarifying under which conditions the policy intervention will improve outcomes.

\section{Conceptual Framework}

This study was concerned with long-term functional status outcomes in the injured population. Specifically, it investigated post-injury health trajectories of individuals who participated in the Health and Retirement Study, a longitudinal panel study that surveys individuals over the age of 50 every two years. This study also investigated the role of health insurance and medical care in determining functional outcomes. The conceptual framework informing this study drew from the Minnesota Center for Health Trajectory Research (MCHTR) Framework (Wyman \& Henly, 2011), Wilson and Cleary's Patient Outcomes Model (Wilson \& Cleary, 1995), and Hadley's Conceptual Model of the Relationships between Health Insurance, Medical Care Use, Health, Education, and Income (Hadley, 2003).

\section{Models of Health Trajectory as a Health Outcome}

Health trajectories represent the pattern of health over time and can be used to describe the dynamic course of health and illness (Henly, Wyman, \& Findorff, 2011). The MCHTR framework adapted the Healthy People 2010 determinants of health model through the addition of a time line in order to account for temporal dimensions of both health and health determinants (Figure 1-1)(Wyman \& Henly, 2011).

Health trajectories can be used to model any type of dynamic health phenomena including developmental and aging processes, the course of specific illnesses and treatments, or pathways leading to a discrete event such as disablement or death. This study builds off the MCHTR framework's assumption that both health determinants and health change over time and that changes in health can occur naturally or be induced clinically (Henly et al., 2011).

Acute Illness vs. Chronic Illness Trajectory. Approaches to studying illness over time often differ for acute illness and chronic illness. Henly et al. (2011) describe acute illness trajectories as being associated with illnesses that have a sudden onset and short duration such as emergency events (e.g. myocardial infarction, trauma), surgical interventions, acute exacerbations of chronic diseases, and time-delimited medical treatments. It is typically assumed that acute illnesses adhere to predictable patterns and that medical treatment will resolve or attenuate symptoms. Chronic illness trajectories are 


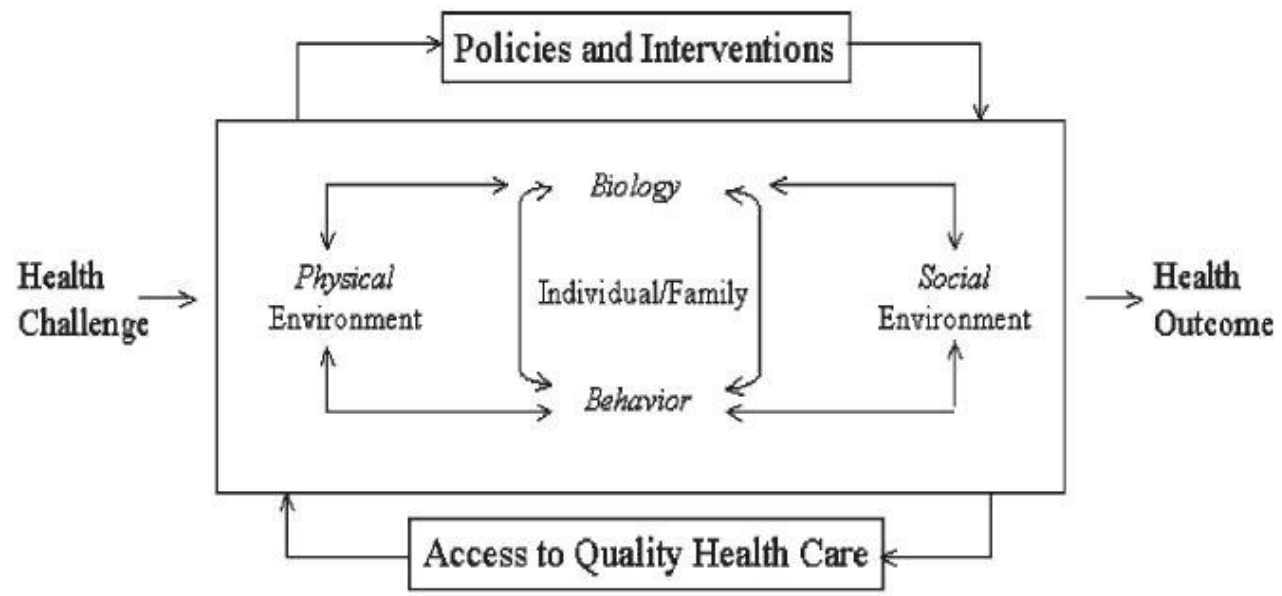

Time

Figure 1-1. Minnesota Center for Health Trajectory Research Framework

Reprinted with permission from Wyman JF, Henly SJ. (2011). Advancing nursing science through health trajectory research: an introduction. Nurs Res 60(3 Suppl):S1-4. 
those associated with acute events that result in irreversible damage (e.g. stroke, spinal cord injury) and lasting or progressive diseases such as diabetes and Parkinson's disease. Chronic illness trajectories assume that medical treatment can manage and slow the progression of chronic conditions; however the underlying pathology cannot be eradicated (Wyman \& Henly, 2011).

Because this study followed individuals after an acute event, it incorporates assumptions from both acute and chronic illness models. It assumes that it is possible for medical treatment to resolve or mitigate post-injury functional deficits, however, it is noted that individual illness trajectories will differ and some participants may follow a trajectory more similar to that of chronic illnesses. Because the event being studied is heterogeneous in nature, the irreversibly and extent of an injury's damage is dependent upon its severity and location as well as the injured individual's access to resources, preexisting health status, and functional needs. It was therefore assumed that some study participants would follow a trajectory similar to acute illness whereas others would follow a trajectory that parallels that of a chronic illness. A goal of this study was to assess the role of medical care use in modifying health outcomes across and between different outcome trajectories. Therefore, this study assumed that the efficacy of medical treatment can differ depending on whether an individual follows a trajectory of recovery or dysfunction after injury.

Trajectories of Recovery and Dysfunction after Acute Illness. Based on the findings of Woon and colleagues' study investigating cognitive function in survivors of critical illness, Theodore Iwashyna postulates that there are five prototypical trajectories of recovery or dysfunction after acute illness (Woon, Dunn, \& Hopkins, 2012; Iwashyna, 2012). These hypothesized trajectories informed this study by providing descriptive categories of functional status trajectory outcomes.

The first type of trajectory described is that of patients who have no impairment at discharge and remain unimpaired 6 months later. The second trajectory is similar to the first, however it describes patients who have significant impairments at hospital discharge and at 6 months follow-up, suggesting that these patients "get knocked down and stay down". The literature has focused heavily on patients who experience this type of trajectory and extensive research has been done on long-term outcomes in patient populations that have suffered severe neurological injuries.

Iwashyna (2012) describes three other dynamic trajectories based on Woon's results: "The Big Hit" (Figure 1-2), "The Slow Burn" (Figure 1-3), and "Relapsing Recurrences" (Figure 1-4) trajectories (Iwashyna 2012). Iwashyna (2012) describes the Big Hit trajectory as the implicit mental model of recovery held by clinicians where injured patients experience an acute loss of function after injury that is followed by gradual recovery. This model assumes that the depth of the initial functional loss and measures of functional deficit at discharge are predictive of functional status at 6 months. Surprisingly, Woon et al. (2012) found that cognitive function at discharge was not significantly associated with cognitive function at 6-month follow-up and only $28 \%$ of patients exhibited the Big Hit pattern of recovery (Woon et al., 2012). Iwashyna (2012) 


\section{The Big Hit}

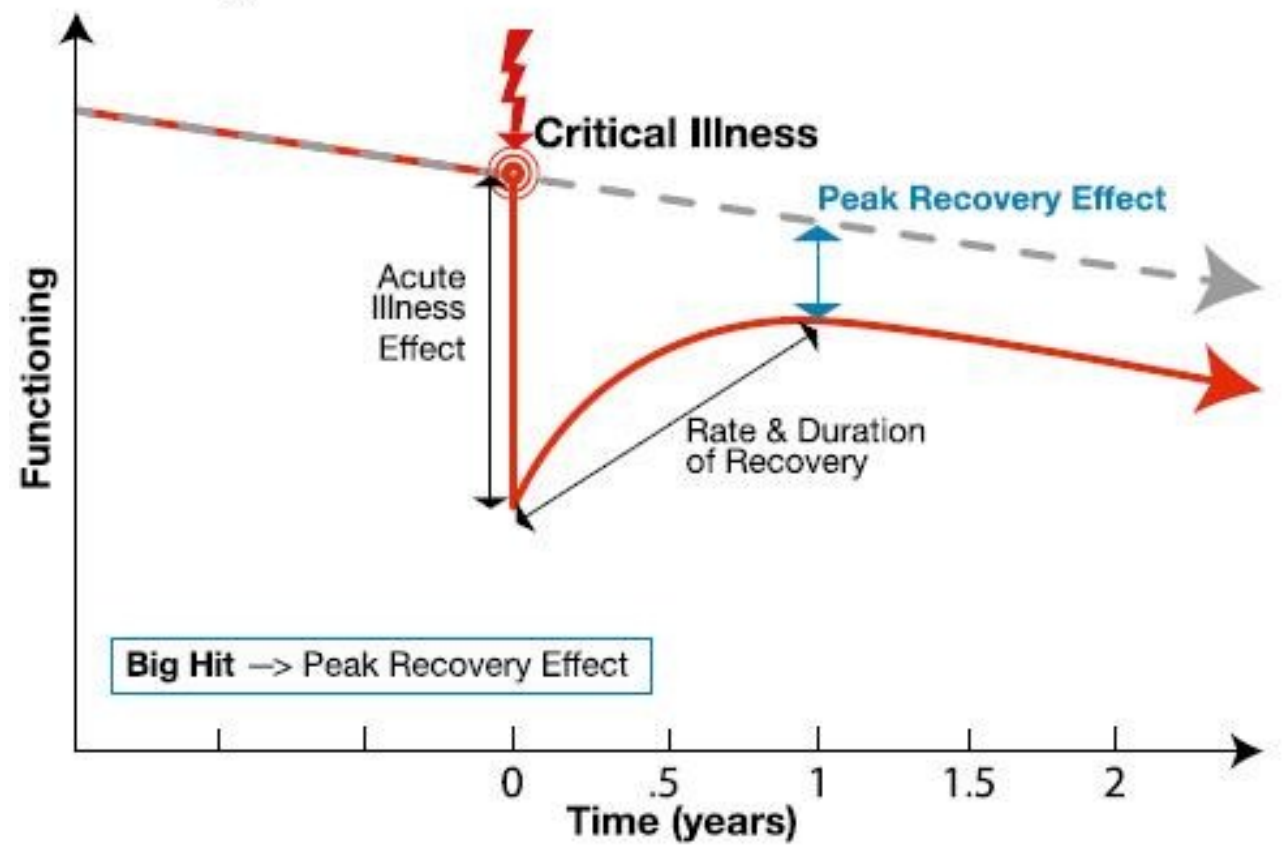

Figure 1-2. Iwashyna's Big Hit Trajectory of Recovery after Acute Illness

Reprinted with permission of the American Thoracic Society. Copyright (C) 2014 American Thoracic Society. Iwashyna TJ. (2012). Trajectories of recovery and dysfunction after acute illness, with implications for clinical trial design. Am J Respir Crit Care Med 15;186(4):302-4. Official Journal of the American Thoracic Society. 


\section{The Slow Burn}

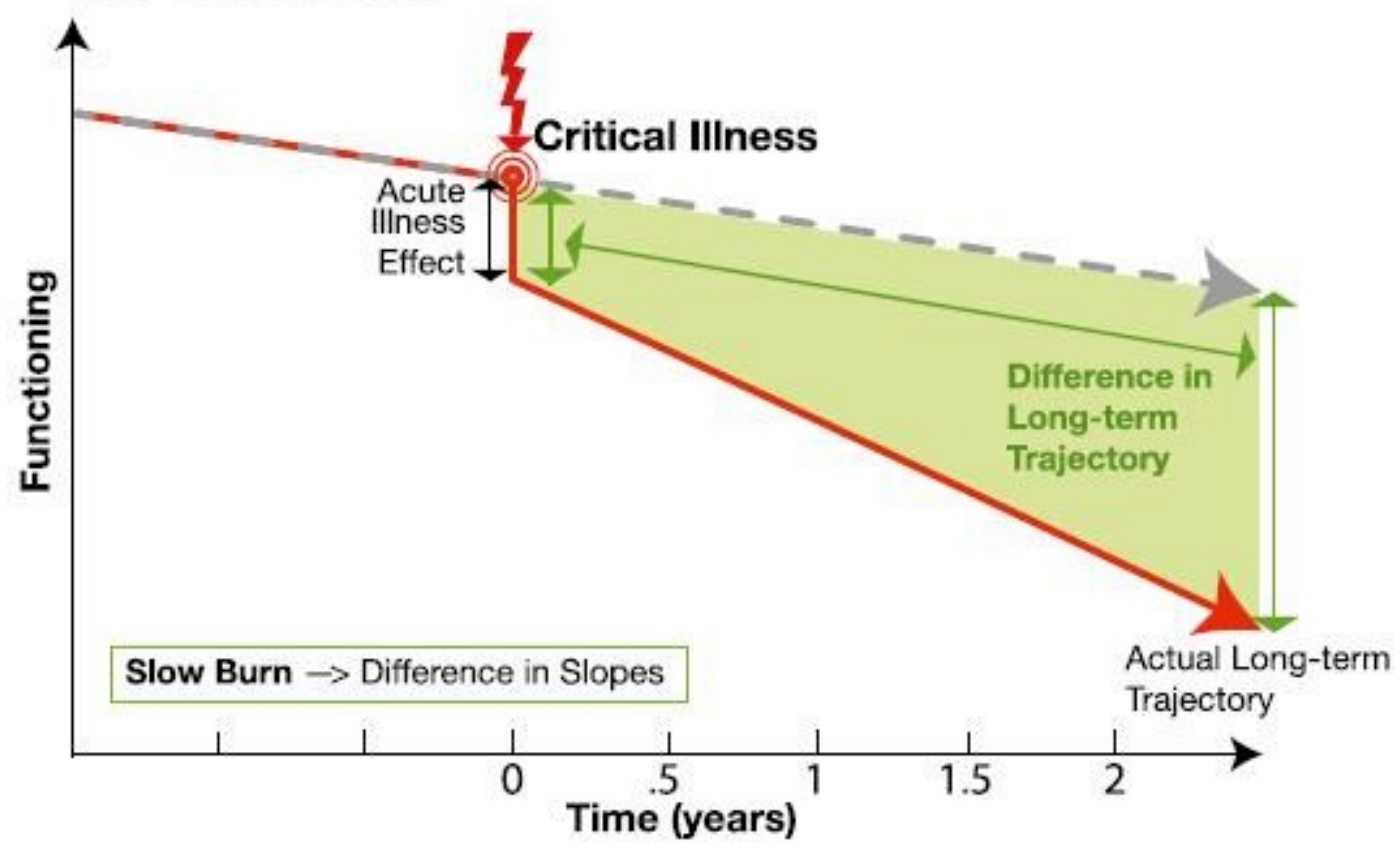

Figure 1-3. Iwashyna's Slow Burn Trajectory of Recovery after Acute Illness

Reprinted with permission of the American Thoracic Society. Copyright (C) 2014 American Thoracic Society. Iwashyna TJ. (2012). Trajectories of recovery and dysfunction after acute illness, with implications for clinical trial design. Am J Respir Crit Care Med 15;186(4):302-4. Official Journal of the American Thoracic Society. 


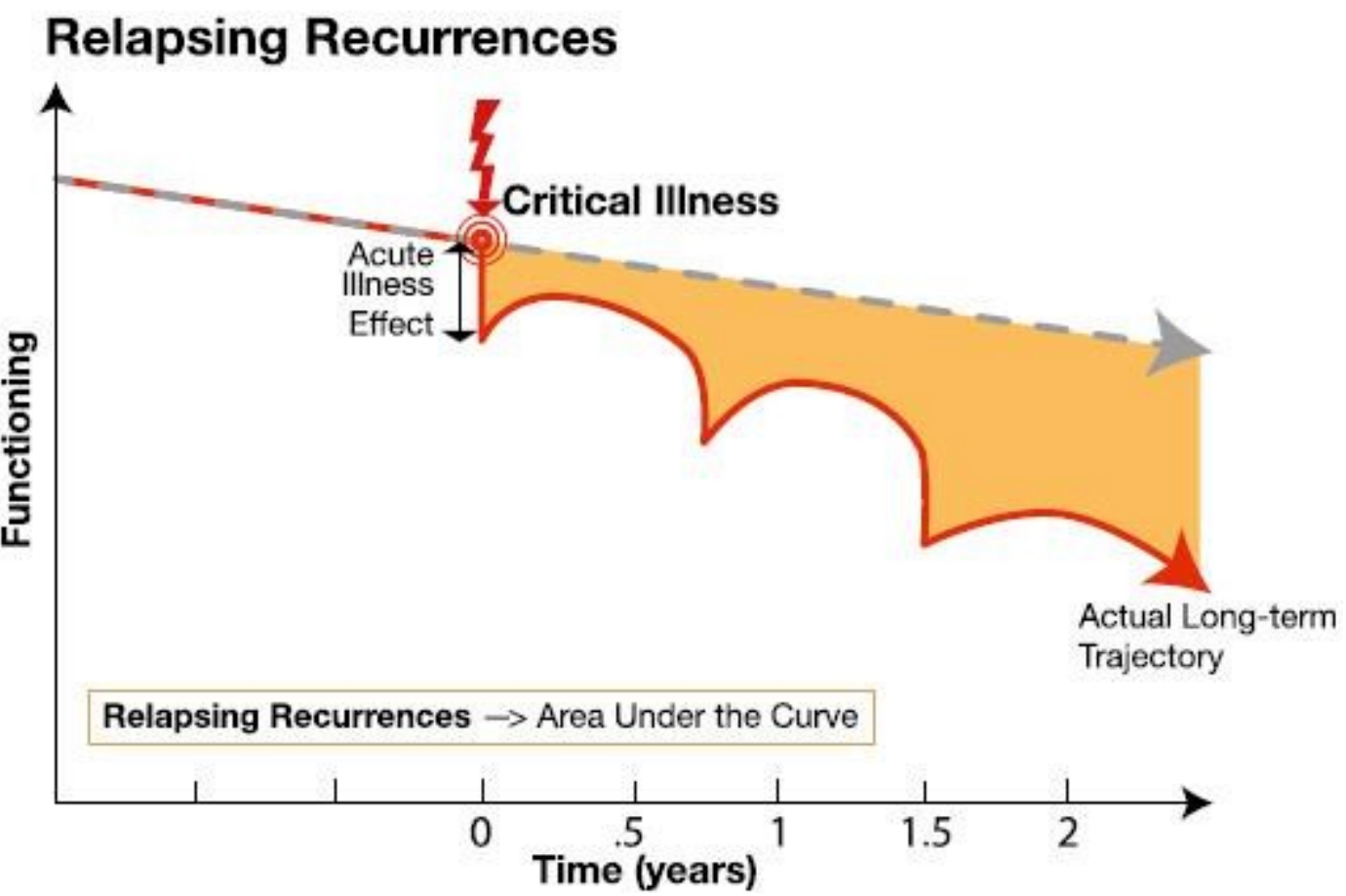

Figure 1-4. Iwashyna's Relapsing Recurrences Trajectory of Recovery after Acute Illness

Reprinted with permission of the American Thoracic Society. Copyright (C) 2014 American Thoracic Society. Iwashyna TJ. (2012). Trajectories of recovery and dysfunction after acute illness, with implications for clinical trial design. Am J Respir Crit Care Med 15;186(4):302-4. Official Journal of the American Thoracic Society. 
argues that characteristics of the population that follows this recovery trajectory should be examined and actions taken by these patients, their caregivers, and their medical teams should be studied to determine if there are any contributors to recovery that can be generalized to the broader population (Iwashyna, 2012).

The next two trajectories, "The Slow Burn" and "Relapsing Recurrence," reflect what $23 \%$ of the patients demonstrated in the Woon study. These trajectories describe patients who had no functional impairment at discharge but were significantly cognitively impaired at the 6-month time point. In the Slow Burn trajectory, patients are sent home from the hospital and experience a persistent and rapid decline in functional status. The Relapsing Recurrence trajectory is characteristic of patients who have acute exacerbations followed by partial recovery.

These hypothesized trajectories provide a framework for investigating clinicallymeaningful and trajectory-specific long-term outcomes in the injured population. Because patients' long-term outcomes after injury vary, it is necessary to plot trajectories of the outcome of interest so that the nature and relative frequency of each recovery subtype can be determined. Iwashyna argues that each trajectory requires investigators to identify an end point and an outcome that corresponds to the subtype's functional form. For example, patients on a Big Hit trajectory should be followed until maximal recovery is reached and the magnitude of the residual deficit should be measured. However, patients on the Slow Burn and Relapsing Recurrence trajectories do not have a single time point at which the change in absolute level of function should be measured. Instead, studies should follow such patients over multiple time points and focus on measurements that capture change in the trajectory of decline (Iwashyna 2012). This study incorporated these insights by using data collected at multiple time points so that variables influencing changes in trajectory could be identified.

\section{Determinants of Functional Status after Injury}

Health Status and Socioeconomic Status. Just as the MCHTR framework adapts the Healthy People 2010 framework by incorporating a temporal dimension into its models, this study utilized Wilson and Cleary's Patient Outcomes Model to identify determinants of functional status that undergo change over time. Wilson and Cleary propose that functional status is determined by biological and physiological variables, symptom status, individual characteristics, and environmental characteristics such as social and economic support (Figure 1-5) (Wilson \& Cleary, 1995).

The arrows in the Wilson and Cleary model indicate causal relationships. The framework for this study considered measurements of biological and physiological variables and symptom status to fall under a single latent construct called Health Status. Wilson and Cleary (1995) claim that although variables relating to health status are highly correlated with functioning, they do not fully explain all variation. One study

carried out by Cleary et al (1993), examined functioning in HIV patients and found that sociodemographic variables explained $25-39 \%$ of variation in physical functioning 


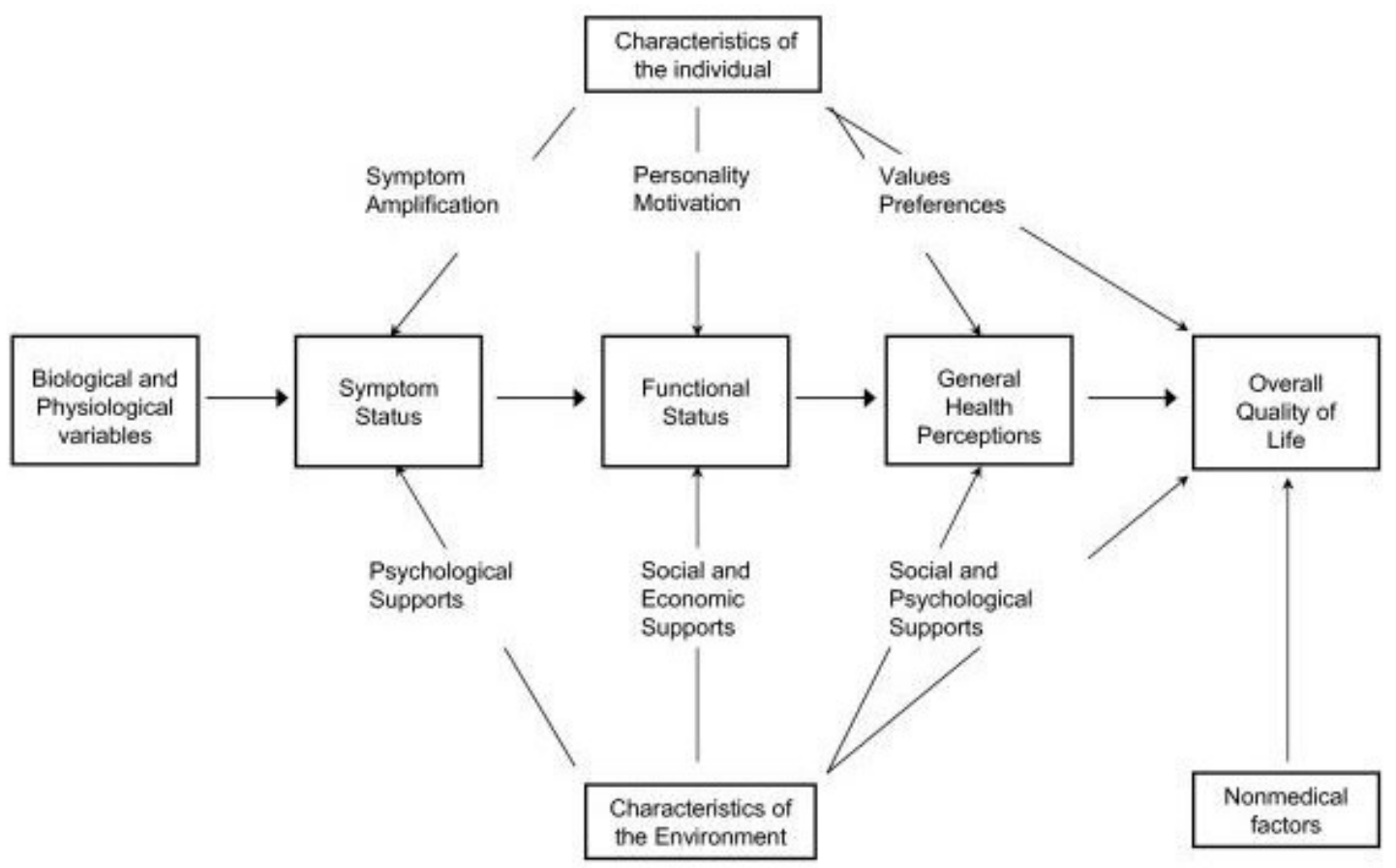

Figure 1-5. Wilson and Cleary's Patient Outcomes Model

Reprinted with permission from Wilson IB, Cleary PD. (1995). Linking clinical variables with health-related quality of life. A conceptual model of patient outcomes. JAMA 4;273(1):59-65. 
whereas health-related variables explained 55\% (Cleary et al., 1993). The proposed framework incorporates a latent construct for individual socioeconomic status that combines measurements of income, assets, and educational attainment. It also uses sociodemographic factors such as age, gender, race/ethnicity, and social support as covariates in the model. Measurements of activities of daily living and independent activities of daily living are combined to form a latent construct of functional status. Health status, socioeconomic status, and the sociodemographic covariates are assumed to have a causal and direct relationship to functional status.

Insurance Status and Medical Care Use. Aside from health-related and socioeconomic factors, medical factors will also be taken into account in modeling functional outcomes. Because this study is interested in identifying how health insurance status affects injury outcomes, insurance status will be included in the model. Hadley (2003) hypothesizes that health insurance influences both the quantity and quality of medical care used and medical care goes on to influence health. He also goes on to state that health status influences education, work, and income, which loop back and affect health insurance and medical care use (Figure 1-6) (Hadley, 2003).

Conceptually, Hadley's framework is significantly more complex than Wilson and Cleary's due to the presence of feedback loops. However, it is important to account for endogenous relationships when modeling the effects of health insurance and medical care use on health outcomes. Furthermore, Hadley notes that both health insurance and medical care use are multidimensional constructs with important temporal components. For example, health insurance can cover a broad or narrow set of services and have small or large patient cost-sharing obligations. Additionally, the effects of insurance coverage and medical care use may take years to manifest themselves and could also be cumulative (Hadley, 2003).

Similar to Wilson and Cleary's framework, the effects of health status, socioeconomic status, insurance status, and medical care use on functional status will be considered unidirectional. Health insurance status will be measured by primary plan type (i.e., uninsured, public, and private). Due to the multidimensional nature of medical care use, this construct will consist of multiple measured variables. Medical care use will be measured by outpatient doctor visits, prescription drug usage, and outpatient surgery. Utilization of inpatient services is not included in the medical care use construct since hospitalization is conceptually related to an adverse health event and is associated with a decline in functional status. The model for this study is interested in assessing the impact of medical care that is associated with management and improvement of functional status.

\section{Conceptual Model for the Proposed Study}

Figure 1-7 displays the conceptual model for the study. It recognizes that both medical care use and functional status are a function of patient health status, insurance 


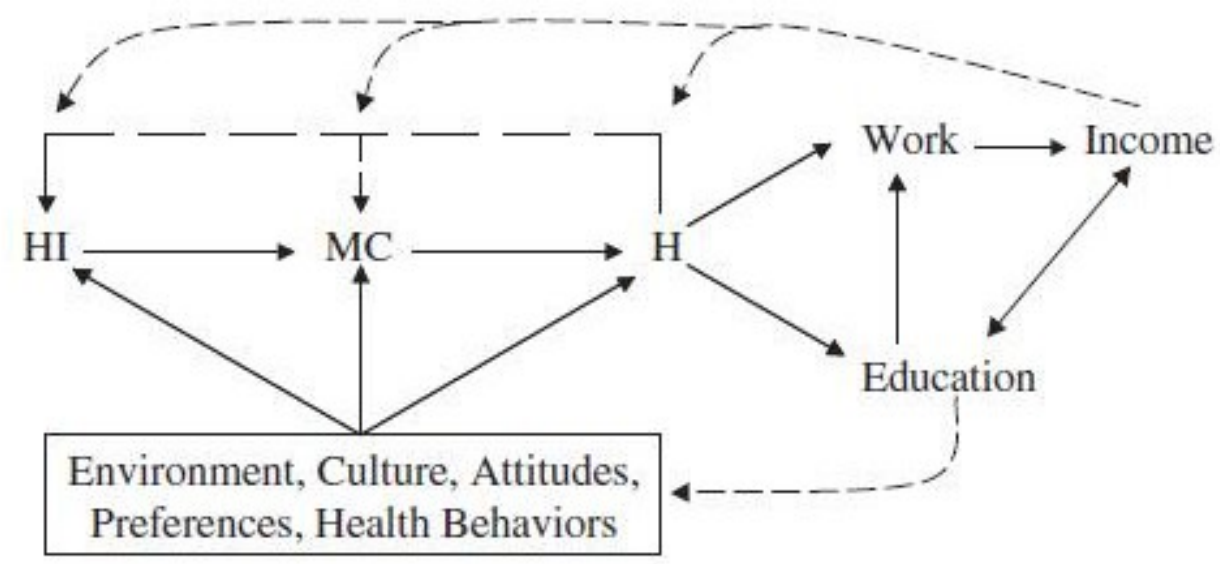

HI - Health Insurance MC - Medical Care H - Health

\section{Figure 1-6. Hadley's Framework}

Reprinted with permission from Hadley J. (2003). Sicker and poorer--the consequences of being uninsured: a review of the research on the relationship between health insurance, medical care use, health, work, and income. Med Care Res Rev 60(2 Suppl):3S-75S. 


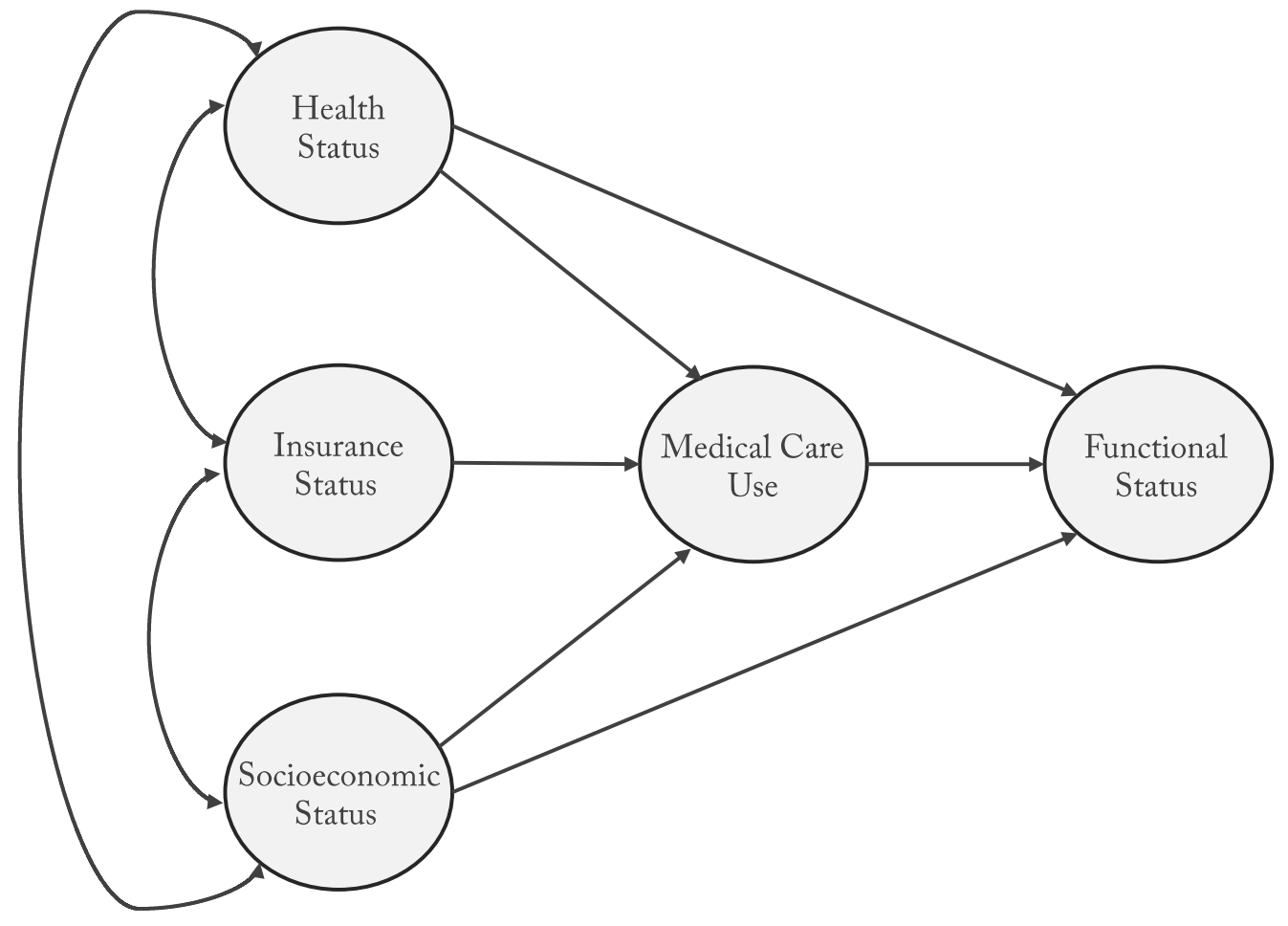

Figure 1-7. Conceptual Model for the Study 
status, and socioeconomic status. It also accounts for reciprocal relationships between predictor variables.

\title{
Specific Aims and Hypotheses
}

This study aimed to model distinct functional status trajectories after injury. The literature demonstrates that long-term functional deficits persist after injury. However, a thorough analysis of how outcomes vary overtime between individuals in the all injury population has not been conducted. This study also aimed to determine how insurance status and medical care use work together to define outcomes. Studies have extensively documented that insurance status is one of the strongest predictors of outcome after injury, yet the causal mechanism by which insurance exerts its effect is not described. It is possible that the significance of insurance status' role in defining injury outcomes is due to it serving as an indicator for an omitted variable, such as socioeconomic status. However, it is more likely that insurance status works through a mediating variable, such as medical care use, in order to influence post-injury outcomes. Investigating the process underlying how insurance status modifies functional outcomes provided information that is useful in designing effective policy interventions.

This study was divided into two parts (1) identifying injury subgroups at-risk for enduring post-injury functional deficits and (2) assessing the impact of health insurance and medical care use on long-term changes in functional status.

\author{
Aim 1 \\ To classify subgroups with distinct recovery patterns within the injured \\ population and to identify individual characteristics that predict recovery after injury. \\ - Hypothesis 1: Individuals who are injured will have variation in functional status \\ trajectories that can be classified into recovery subgroups. \\ - 1a: Trajectory analysis will reveal a subgroup that has no functional \\ limitations at the time of injury and remains limitation-free for the \\ duration of the study \\ - 1b: Trajectory analysis will reveal a subgroup that has significant \\ functional limitations at the time of injury and limitations remain for the \\ duration of the study \\ - 1c: Trajectory analysis will reveal a subgroup that has functional \\ limitations after injury and then returns to near-baseline levels of \\ functioning throughout the post-injury period \\ - 1d: Trajectory analysis will reveal a subgroup that has functional \\ limitations at the time of injury and then continues to develop more \\ limitations throughout the post-injury period
}


- 1e: Trajectory analysis will reveal a subgroup that has fluctuating levels of functional limitations over the course of the study.

- Hypothesis 2: Pre-injury insurance status will be predictive of an individual's recovery subgroup.

- 2a: Private insurance will be predictive of functional trajectories with fewer functional limitations

- 2b: Public insurance will be predictive of functional trajectories with more functional limitations

- 2c: Having no insurance will be predictive of functional trajectories with more functional limitations

\begin{abstract}
$\operatorname{Aim} 2$
To determine whether medical care use mediates the relationship between insurance status and long-term functional status after injury.

- Hypothesis 3: Medical care use will mediate the influence of insurance status on long-term functional outcomes after injury.

- 3a: Outpatient doctor visits will mediate the effect of insurance status on functional outcomes

- 3b: Prescription drug use will mediate the effect of insurance status on functional outcomes

- 3c: Outpatient surgery will mediate the effect of insurance status on functional outcomes
\end{abstract}

\title{
Definition of Terms
}

Following are key terms used in the study and their definitions:

- Functional Limitations - difficulty performing tasks or activities as a result of a functional impairment.

- Latent Class - a term referring to a subpopulation in the context of unobserved heterogeneity; the term 'latent class' is used when homogenous subpopulations within a heterogeneous population are unknown prior to analysis and must be inferred from the data.

- Medical Care Use - in this study, medical care use refers to the utilization of outpatient services, procedures, or medications. It does not incorporate measures of acute care.

- Relative Effects - in the mediation analysis, the total, direct, and indirect effects for a given level of insurance status is reported in relative to a reference level of insurance status. This study examined the effects of public insurance and no 
insurance relative to private insurance.

- Subpopulation - a term referring to a cluster of individuals with similar characteristics within a heterogeneous population.

- Trajectory - the course of an outcome over time.

- Wave - the time point at which a survey was administered. This study analyzed six waves of data from the Health and Retirement Study collected between 1998 and 2008. Data was collected in two year intervals. Wave 1 takes place in 1998 and is referred to as the pre-injury period. Wave 2 takes place in 2000 and is referred to as the peri-injury period. Waves 3, 4, 5, and 6 take place during 2002, 2004,2006 , and 2008, respectively. These waves were collectively referred to as the post-injury period in the study. 


\section{CHAPTER 2. REVIEW OF THE LITERATURE}

\section{Introduction}

This chapter is organized into three parts in order to provide information from the current literature on (1) long term functional outcomes after injury and individual predictors of post-injury functional status, (2) the role of medical care use in recovery after injury, and (3) studies with methodological approaches similar to the ones used in this study. This review begins by introducing the concept of functional status and inventorying common functional status indicators. Next, it reports findings from studies that examine the duration of injury's impact on individual functioning and that document individual-level predictors of functional outcomes.

This chapter also aims to summarize literature that examines the utilization of medical services in acute, post-acute, and ambulatory care settings in relation to injury outcomes. This study hypothesizes that post-injury outcome disparities associated with insurance status arise due to differences in access, quantity, and comprehensiveness of provided medical care. In other words, regulation of medical care use is the proposed mechanism by which insurance status determines post-injury outcome. Studies assessing the relationship between insurance status and medical care use after injury are therefore evaluated. Finally, this chapter concludes by characterizing the complex research gap existent within the field of injury research and describing how this study addresses prevailing gaps in the literature.

\section{Functional Status}

The main outcome in this study is long-term functional status after injury. Functional status is "an individual's ability to perform normal daily activities required to meet basic needs, fulfill usual roles, and maintain health and well-being" (Leidy, 1994). Functional status encompasses both functional capacity, an individual's maximum capacity to perform daily activities in the physical, psychological, and social domains of life; and functional performance, the activities people do during the course of their daily lives. The loss of independence in activities of daily living results in a decline in functional status. Changes in functional status can be brought on by natural processes such as aging or can be induced by the onset of a health condition.

\section{Components of Functional Status}

The World Health Organization's International Classification of Functioning, Disability and Health (ICF) describes two functioning components that can be directly influenced by a person's health condition: 1) body functions and body structures and 2) activities and participation (World Health Organization [WHO], 2001). Body functions

and structures relate to physiological and anatomical characteristics of an individual 
whereas activity and participation relate to an individual's context in regards to the activities and roles they need to perform. The ICF also includes an environmental component that takes into account whether or not a person's environment is conducive to performing daily activities. Therefore, functional status is determined by a person's physical ability, their participation in activities, and environmental factors.

When health conditions such as injury adversely affect either functioning component, disability results. Deficits in body function and structure are identified as impairments, difficulties in performing tasks or activities are considered activity limitations, and problems with social participation are defined as participation restrictions (Tomey \& Sowers, 2009). Disability arises when impairments, activity limitations, and participation restrictions deprive individuals from having a full range of functional ability when contextual factors are not supportive. Contextual factors refer to characteristics of an individual's physical and social environment. A person's context often determines whether or not functional ability is diminished. Therefore, overall functional status is determined by the interaction between an individual's capacities and their contextual factors (WHO, 2001) (Figure 2-1). This study focuses on activity limitations in its evaluation post-injury functional status.

\section{The Relationship between Functioning and Disability}

Injury research is primarily concerned with assessing the extent of injury's impact on individual functioning and whether or not individuals go on to experience disability due to changes in functional status. The WHO's International Classification of Impairments, Disabilities and Handicaps (ICIDH) proposes a framework that describes disability according to three dimensions: impairment, disability, and handicap (WHO, 1980). An impairment is "any loss or abnormality of psychological, physiological or anatomical structure or function" (WHO, 1980). Impairment occurs at the level of organ or system function and is assessed by determining if the body is functioning according to accepted standards. A disability is "any restriction or lack (resulting from an impairment) of ability to perform an activity in the manner or within the range considered normal for a human being" (WHO, 1980). Disability refers to limitations in functional performance or activities that affect the whole person. Lastly, a handicap is "a disadvantage for a given individual, resulting from an impairment or a disability that limits or prevents the fulfillment of a role that is normal (depending on age, sex, and social and cultural factors) for that individual" (WHO, 1980). The dimension of handicap considers how an individual with a disability interacts and adapts to their environment and whether or not that individual experiences a disadvantage in relation to their peers due to a disability.

Although this study is primarily concerned with functional limitations as an outcome rather than disability, the ICIDH framework provides important distinctions in regards to the consequences of bodily injury. For example, nearly all participants with an injury will experience an impairment that may or may not be permanent. If the impairment causes interference of task functions, then disability results (Griffin, 2002). However, disability could be eliminated through the use of assistive equipment since the 


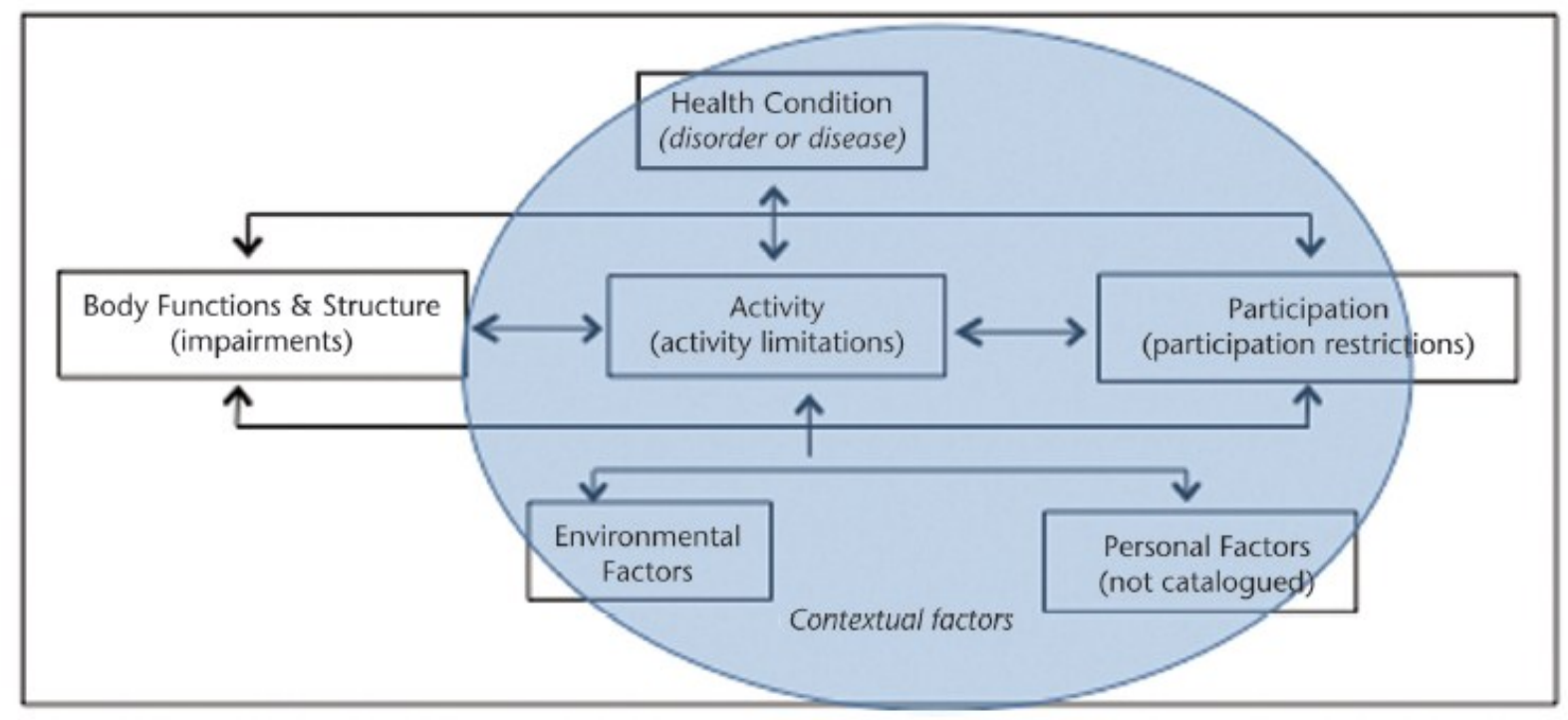

\section{Figure 2-1. International Classification of Functioning, Disability, and Health}

Reproduced with permission from the World Health Organization. (2001). The International Classification of Functioning, Disability and Health. Available at http://www.who.int/classifications/docs/en/ 
equipment would allow for the person to carry out an activity they would otherwise not be able to do. Individuals with similar impairments could differ in regards to the functional limitations they experience due to differences in access to resources such as social support and medical care as well as other contextual factors. Therefore, the functional consequence of an injury is only partially determined by the physical impairment it induces.

\section{Functional Status Indicators and Associated Functional Domains}

Individual functioning, the ability to perform normal daily activities required to meet basic needs, fulfill usual roles, and maintain health and well-being, can be measured using functional status indicators (FSI), which "capture the impact of a health condition on an individual, independent of the particular health condition" (Mayo et al., 2005, p. 1195). Conceptually, functioning is operationalized as the extent to which an individual can carry out necessary or desired activities (McDowell, 2006). It follows that some aspects of functioning may be more relevant to health research than others as human functioning is an expansive concept. Stewart et al list six characteristics that measures of functioning should possess in order to be clinically meaningful and useful in investigating quality of medical care:

(1) the set of measures should be comprehensive in terms of the outcomes that are important to patients; (2) measures must be short and easy to administer; (3) patients with chronic conditions should score lower on the measures than patients with no chronic condition; (4) scores on each health component should correspond to specific features of each disease (e.g., those with arthritis should have more pain than those with hypertension); (5) within each condition, scores should vary for patients whose conditions differ in severity at a point in and over time; and (6) measures should be sensitive to the beneficial or harmful effects of treatments over time (Stewart et al., 1989, p. 1-2).

Additionally, for the purposes of this study, functional status indicators should be valid for comparing outcomes across groups with various types of injury.

As a concept, functional status encompasses multiple types of functioning which are broadly divided into physical, psychological, social, and occupational domains (Kendrick et al., 2011). Functional domains have a certain degree of overlap and studies can potentially vary in regards to the domain a given indicator is assigned to and which domains are explicitly studied. Additionally, researchers may include more than four functional domains by expanding the scope of indicators that are measured or refining categories so that each domain contains a more specific set of related indicators. The indicators a study measures and the domains it assesses are typically determined by the research instrument chosen for the study. A description of four major functional domains and their commonly associated functional status indicators are provided below. 
Physical Functioning. Physical functioning is comprised of an individual's physical capacity as well as their overall level of vitality and freedom from bodily pain or discomfort. Physical functioning often includes measures of activities of daily living (ADL), dependence on medication and medical aids, levels of energy and fatigue, presence of bodily pain, and quality of sleep (WHO, 1996).

One of the most commonly used indicators of physical functioning is ADL. Five categories of ADL include 1) self-care (feeding, bathing, dressing), 2) mobility (getting around indoor and outdoor environments or the community), 3) physical activities (walking, using stairs, lifting, bending), 4) role activities (work, school, household, and social settings) and, 5) leisure activities (hobbies, sports, recreation) (Stewart, Ware, \& Brook, 1981). ADL are commonly broken into two groups, basic activities of daily living (BADL) and instrumental activities of daily living (IADL). BADL relate to tasks that involve self-care such as personal hygiene and grooming, dressing and undressing, selffeeding, functional transfers (e.g. getting into and out of bed), bowel and bladder management, and ambulation (McDowell, Engberg, Rodriguez, Engberg, \& Sereika, 1996). IADL consist of activities that allow an individual to live independently but are not required for fundamental functioning. Performing housework, taking medication as prescribed, managing money, shopping for groceries, using the telephone, using technology, and transportation within the community are all examples of IADL (Bookman, Harrington, Pass, \& Reisner, 2007).

Other measures of physical functioning assess sensory functions such as seeing or hearing and whether or not impairments cause difficulty with reading or conversation (McDowell, 2006). Indicators such as bodily pain when performing everyday activities, fatigue or lack of energy, and difficulty sleeping reflect problems in physical functioning, however, these measures are also related to the domain of psychological functioning.

Psychological Functioning. Psychological functioning includes emotions and cognitions. Emotional functioning includes the perception, use, understanding, and management of emotion whereas cognitive functioning includes thinking, learning, memory, attention, and decision-making (Brackett, Rivers, Shiffman, Lerner, \& Salovey, 2006). Studies commonly assess emotional and cognitive functioning separately. Indicators of emotional function typically relate to the frequency and severity that someone experiences feelings of depression, anxiety, and frustration. An individual's ability to cope with stress or experience motivation may also be evaluated (McDowell, 2006). Studies may also evaluate emotional functioning by asking participants about psychological outcomes such as post-traumatic stress disorder, depression, and anxiety or behavioral outcomes such as alcohol and illicit drug use (Michaels et al., 2000; Sluys, Häggmark, \& Iselius, 2005).

Cognitive functioning indicators are associated with an individual's ability to think. Items such as problem-solving capabilities, judgment and reasoning, orientation, and memory relate to cognitive functions. Additionally, components of attention span such as distractability, concentration, and levels of alertness and responsiveness are also included in this domain (McDowell, 2006). Difficulty understanding the plot of a TV 
show, remembering appointments, or playing a game of skill such as chess are indicative of reduced cognitive functioning.

Social Functioning. Social functioning refers to an individual's ability to interact in the normal or usual way in society. Personal and working relationships as well as social support are related to the domain of social functioning (WHO, 1996). Social support refers to "exchanges of social, emotional, and instrumental resources between the individual and his or her social environment, intended to enhance the well-being of the individual concerned" (Suurmeijer, Reuvekamp, \& Aldenkamp, 2002). Indicators of social functioning include the frequency of conflicts in an individual's relationships, frequency and initiation of social contact, and appropriateness of behavior in relationships (McDowell, 2006). Indications that an individual is having problems with social functioning include difficulty interacting with co-workers at work, anxiety in dealing with people, and isolating oneself from others (Brissos, Balanzá-Martinez, Dias, Carita, \& Figueira 2011).

Occupational Functioning. Occupational functioning relates to an individual's employment, workplace performance, and productivity. This domain has considerable overlap with physical, psychological, and social functioning. For example, effective performance at work often requires high psychological functional ability so that workers have the capacity for time management, self-organization, planning and problem-solving, and self-motivation (Barkley \& Murphy, 2010). Assessments of occupational functioning may ask participants about their ability to tolerate planned number of work hours in a day, their capacity to perform necessary job functions, and vocational placement (McDowell, 2006). Individuals that struggle with occupational functioning may have difficulty keeping a job, miss more days of work, receive poor evaluations, be promoted less often, change jobs more frequently, or be underemployed (Erbes, Kaler, Schult, Polusny, \& Arbisi, 2011).

The outcome of this study is functional limitations. Limitations can arise due to functional deficits in any of the described domains. However, physical deficits are most likely to have the greatest impact on the outcome measured in this study.

\section{Long-term Functional Outcomes after Injury in Adults (2+ Years Post-injury)}

To date, the majority of studies examining injury outcomes have been short-term, investigating functional changes that occur in patients up to two years after injury. Furthermore, most of this research has focused on recovery that takes place during the six-month period immediately following injury. Despite this, several long-term studies have been conducted and found that residual functional deficits persist beyond two years post-injury. Long-lasting effects of injury have been reported to affect all major functional domains including physical, psychological, social, and occupational functioning. 
Change in physical functioning has been strongly associated with injury. Physical functioning is comprised of an individual's physical capacity as well as their overall level of vitality and freedom from bodily pain or discomfort. Physical functioning often includes measures of activities of daily living (ADL), dependence on medication and medical aids, levels of energy and fatigue, presence of bodily pain, and quality of sleep (WHO, 1996). Deterioration in mobility, self-care, usual activities, and pain were reported in several studies examining outcomes 2-7 years after injury (Malt, Blikra, \& Høivik, 1989; Butcher et al., 1996; Keogh, Nuwayhid, Gordon, \& Gucer, 2000; Harris, Young, Rae, Jalaludin, \& Solomon, 2006; Ulvik, Kvåle, Wentzel-Larsen, \& Flaatten, 2007; Sluys et al., 2005; Mackenzie et al. 2005; Castillo, MacKenzie, Wegener, \& Bosse 2006; Rivara et al. 2008; Livingston, Tripp, Biggs, \& Lavery, 2009; Overgaard, Høyer, \& Christensen, 2011; Soberg, Finset, Roise, \& Bautz-Holter, 2012; Derrett et al., 2013). A study examining long-term outcomes in trauma patents found that $68 \%$ of patients reported that they were still suffering from physical disabilities five years after injury (Sluys et al., 2005). A large proportion of injured patients not only report worse physical health two years after injury compared to their pre-injury health status, but many continue to report further declines in physical health beyond the first two post-injury years (Soberg et al., 2012). However, approximately $20 \%$ of patients reported improved physical health between 2 and 5 years post-injury, indicating that a sizeable proportion of the population has a recovery process that spans multiple years (Castillo, Mackenzie, \& Bosse 2011; Soberg et al., 2012).

Changes in physical functioning are also strongly associated with long-term psychosocial functioning in patients (Zatzick et al., 2008). As understanding the impact of injury on quality of life becomes an increasing focus for researchers, more studies have started documenting the consequences of injury on psychological and social functioning. Psychological functioning includes both emotional and cognitive functioning. Emotional functioning includes the perception, use, understanding, and management of emotion whereas cognitive functioning includes thinking, learning, memory, attention, and decision-making (Brackett et al., 2006). Mental health problems including development of depression and/or anxiety, difficulty concentrating and remembering things, low self-esteem, and increased usage of alcohol and tobacco have all been reported as long-term outcomes after injury (Malt et al., 1989, Keough et al., 2000, Mayou \& Bryant, 2002; Sluys et al., 2005; Whitnall, McMillan, Murray, \&Teasdale 2006; Mackenzie et al., 2005; Ulvik et al., 2007; Overgaard et al., 2011, Soberg et al., 2012). In a nationwide U.S. study, it was reported that more than $20 \%$ of injured trauma survivors have symptoms consistent with posttraumatic stress disorder 12 months after discharge (Zatzick et al., 2007). Social functioning, an individual's ability to interact in the normal or usual way in society, is also impacted by injury (Sluys et al., 2005; Overgaard et al., 2011). Reduced participation in society, increased social deprivation, decreased number of friends have all been reported to be significantly higher in patients 5 years post-injury compared to their previous baseline (Pfeiffer et al., 2011; Soberg et al., 2012).

Lastly, injury also significantly impacts long-term occupational functioning. Occupational functioning relates to an individual's employment, workplace performance, 
and productivity. This domain has considerable overlap with physical, psychological, and social functioning. Time off work, unemployment, early retirement, and disability have all been linked to injury (Malt et al., 1989; Keogh et al., 2000; Sluys et al., 2005; Redmill, McIlwee, McNicholl, \& Templeton 2006; Andelic et al., 2009; Overgaard et al., 2011). Although return-to-work rates after major injury are high, many have noted that unemployment rates are higher for the injured population (Rhodes, Aronson, Moerkirk, \& Petrash, 1988; Redmill et al., 2006).

Injury researchers studying long-term outcomes have predominantly focused on patients that experience high threat to life injuries (Langley et al., 2011). Studies that have examined long-term outcomes after injury have primarily consisted of the traumatic injury population. Few have researched outcomes of minor and moderate injuries, despite the fact that low threat to life injuries are more numerous than those that require acute care (Langley et al., 2011). Studies that have compared outcomes between patients with injuries of differing severity generally found that common measures of injury severity are not good predictors of functional outcome (Overgaard et al., 2011). This is possibly because measures such as the injury severity score were designed to predict acute mortality (Overgaard et al., 2011). Minor injuries that pose little threat to life are capable of resulting in serious functional limitations and some have reported that functional impairment is more related to the anatomical location of injury rather than its severity (Hu, Wesson, Logsetty, \& Spence 1994; Langley et al., 2011). Therefore, more research should be conducted in the all-injury population in order to better determine the overall burden imposed by injury.

\section{Patient Characteristics that Predict Functional Status after Injury}

Individual pre-injury sociodemographic and health characteristics are associated with post-injury functional outcomes. Patient age, gender, and race are all significant predictors of long-term outcomes. Additionally, pre-existing health status, socioeconomic status, and the presence of social support are also related to post-injury outcomes.

Age is an obvious and well-studied predictor of functional outcomes. Although younger individuals have higher rates of injury, the elderly tend to have worse outcomes due to their frailty. Survivors of injury that are over 65 years of age have been reported to have decreases in physical, emotional, cognitive, and social functioning (Inaba et al., 2003; Sluys et al., 2005; Polinder et al., 2007). Compared to older uninjured individuals, those who are injured are more likely to have limitations in performance of ADL 2 years after injury (McGwin, Melton, May, \& Rue, 2000). Older individuals are also less likely to live independently following injury and more likely to require long-term home care. A Canadian study of injured older adults found that prior to injury $98 \%$ lived independently. Two years after injury approximately only $63 \%$ were living independently and $20 \%$ still required home care (Inaba et al., 2003). The relationship between older age and poorer functional outcome exists even after adjusting for injury severity, comorbidities, and complications (Jacoby, Ackerson, \& Richmond, 2006). 
Gender differences in functional outcomes after injury have also been reported. Women are at significantly higher risk for decreased functioning and lower quality of life after injury compared to men (Holbrook, Hoyt, Stein, \& Sieber, 2001; McGeary, Mayer, Gatchel, Anagnostis, \& Proctor, 2003; Holbrook \& Hoyt, 2004; Polinder et al., 2007). Adverse psychological outcomes are also more common in women including depression, acute stress reaction, and post-traumatic stress disorder (PTSD) (Holbrook et al., 2001; Holbrook, Hoyt, Stein, \& Sieber, 2002; McGeary et al., 2003, Holbrook \& Hoyt, 2004; Aitken, Chaboyer, Kendall, \& Burmeister, 2012; Soberg et al., 2012; Langley et al., 2013). Studies have also found that women are less likely than men to reach full recovery, more likely to suffer disability, and less likely to return to work (Langley et al., 2011; Holbrook \& Hoyt, 2004; Watson, Ozanne-Smith, \& Richardson, 2007; Derrett et al., 2012; Kendrick et al., 2012; Brede, Mayer, \& Gatchel, 2012).

Racial disparities in post-injury functional outcomes have been documented in some studies. Among patients with severe leg injuries, nonwhite race was predictive of poor functional outcomes two years post injury (Bosse et al., 2002). In the traumatic brain injury population, black and Hispanic patients have been reported to have worse physical, social, employment, and disability outcomes than whites (Arango-Lasprilla et al., 2007; Shafi et al., 2007; Gary, Arango-Lasprilla, \& Stevens, 2009; Arango-Lasprilla et al., 2011). Studies examining outcomes in patients with spinal cord injury have found that up to 10 years post-injury, black patients were less likely to be employed than white or Hispanic patients (Arango-Lasprilla et al., 2010). Black patients that suffer spinal cord injury have also been found to report lower subjective well-being scores and fewer hours spent out of bed compared to white patients (Krause, Saladin, \& Adkins, 2009). Because the majority of studies that investigate long-term outcomes after injury have been conducted outside the U.S., race has received relatively less attention in predicting longterm outcomes than other demographic factors.

Health Status prior to injury also plays a role in recovery. Pre-existing comorbidities and mental health status are both associated with patient functional outcomes. Poor mobility, self-care, usual activities, pain, anxiety and depression, and cognitive outcomes are more common in patients with multiple chronic illnesses at the time of injury (McCarthy et al., 1995;Polinder et al., 2007; Langley et al., 2011; Reistetter et al., 2011). Post-injury disability has also been associated with specific comorbidities such as obesity and the presence of two or more chronic illnesses (Lilley, Davie, \& Ameratunga, 2012; Derrett et al., 2012; Langley et al., 2013). Mental health problems that exist prior to injury or that result from injury also prevent patients from returning to optimal functioning. Anxiety, Depression, PTSD, and substance abuse have all been shown to adversely affect recovery and post-injury quality of life (Holbrook, Anderson, Sieber, Browner, \& Hoyt, 1999; Michaels et al., 2000; Holbrook et al., 2001; Kiely, Brasel, Weidner, \& Guse, 2006; Zatzick et al., 2007; Bentler et al., 2009; Wegener, Castillo, Haythornthwaite, Mackenzie, \& Bosse 2011; Castillo et al., 2013).

Socioeconomic Status indicators such as income, education, and profession have been reported to predict functional outcomes after injury. Lower pre-injury income is associated with decreased likelihood of returning to full-time employment, lower 
perceived quality of life, sexual dysfunction, and poor functional outcomes (MacKenzie, Siegel, Shapiro, Moody, \& Smith 1988; Mock et al., 2000; Bosse et al., 2002; Sorensen et al., 2008; Tsaousides et al., 2009; Lilley et al., 2012; Langley et al., 2013). In a study of elderly patients with hip fractures, being in the highest income quintile reduced the amount of decline in functional outcomes such as mobility and motor skills (Bentler et al., 2009). Higher levels of educational attainment are associated significant improvement in functioning and returning to work within the first year (Mackenzie et al., 1988; Mackenzie et al., 1998; Bosse et al., 2002; Mackenzie \& Bosse, 2006; Holtedahl \& Veiersted, 2007; Soberg et al., 2012). Profession is also predictive of outcomes, with white-collar workers being more likely to return to work after injury, less likely to experience disability, and achieve better functional outcomes (Mackenzie et al., 1988; Soberg et al., 2007; Du Bois \& Donceel, 2010; Soberg et al. 2012).

The presence of social support has also been shown to positively affect outcomes. Having supportive friends or family members reduces the likelihood of disability and increases the likelihood of returning to work after injury (Mackenzie et al., 1988; Mock et al., 2000). Social support is also associated with greater functional independence and improved quality of life outcomes (Bosse et al., 2002; Farrell, Bennett, \& Gamelli, 2010; Nijs et al., 2011; Kiely et al., 2006; Erosa, Berry, Elliott, Underhill, \& Fine 2013; Ponsford 2013).

Although several studies have examined predictors of long-term injury outcomes, their generalizability to the U.S. all-injury population is uncertain. Studies on long-term functional outcomes have tended to focus on a narrow subset of the injured population by limiting their study populations to patients with injuries that share a common anatomical location. Predictors of long-term outcomes of traumatic brain and spinal cord injuries are much more commonly studied than other injury types. Given the lifelong consequences of neurological injury, this is to be expected. However, this has produced a body of literature with a restrictive focus on such injury types. As a result, it is difficult to determine the applicability of previous research findings to the general injury population.

Generalizability of previous findings could also be problematic because few longterm studies have been conducted in the U.S. Knowledge concerning predictors of recovery is constrained as previous research has primarily been conducted in European and Commonwealth nations. The U.S. population and healthcare system differ significantly from those of the U.K., Germany, Norway, Sweden, Denmark, Australia, and New Zealand, where much of the research on long-term outcomes has been conducted. The U.S. is possibly more diverse than the countries mentioned and a larger portion of its population is made up of racial and ethnic minorities. Long-term outcomes for groups such as African-Americans and Hispanics, which are associated with health disparities in the U.S., have likely been understudied. Income and educational inequality is also of greater concern in the U.S. Patient socioeconomic characteristics may have more of an impact on long-term outcomes than what has been estimated in European populations. The U.S. healthcare system also differs from those of the European and Commonwealth nations, where citizens' healthcare coverage is essentially universal. Due to privatization of the healthcare system, injured Americans face more barriers to 
accessing needed health services. Lack of healthcare coverage and reduced access to medical care in the United States likely causes difficulties during the recovery process and adverse functional outcomes.

\section{Healthcare-related Predictors of Functional Status after Injury}

Both health insurance and health service utilization are predictive of functional status after injury. Although the effect of health insurance on both in-hospital and longterm mortality has been studied extensively in the injured population, its role in determining long-term functional outcomes is less established. Studies examining the impact of insurance status on functional outcomes have tended to focus on a narrow group of injury types and patient populations. In a U.S. study of patients with severe leg injuries, those without private health insurance were more likely to experience poor functional outcomes two years post-injury (Bosse et al., 2002). In the neurological injury population, insurance type is also associated with differences in functional outcomes (Tate et al.,1994; Pape et al., 2006; Bedell, 2008). Those with private insurance are more likely to return to work or school, achieve better functional outcomes, and have less psychological distress. (Tate et al.,1994; Bedell, 2008). Patients with public insurance were more likely to experience greater handicap and psychological distress after injury compared to privately insured patients despite reporting similar values on measures of functional independence (Tate, Forchheimer, Daugherty, \& Maynard, 1994) Shafi et al. (2007) found that racial disparities in functional outcomes for brain injury patients became insignificant when accounting for health insurance, indicating that insurance status could be responsible for observed differences in outcomes between races (Shafi et al. 2007).

Other studies have examined the role of insurance in the pediatric and elderly populations. In a multisite study investigating quality of life in the pediatric population after traumatic injury, it was found that both Medicaid and being uninsured reduced quality of life scores one year after injury (McCarthy et al., 2006). Because Medicare covers most patients over the age of 65 , studies in the elderly population have primarily compared outcomes in patients covered by insurance plans with different reimbursement strategies. Elderly patients with fee for service insurance plans were more likely to report at two years post-injury that their recovery was going well compared to patients treated through managed care (Keyes, Wickizer, \& Franklin, 2001). Other studies examining hip fractures have reported that both fee-for-service and HMO patients achieve equivalent functional outcomes, however, HMO patients have less intense service utilization (Coleman et al., 2000).

In addition to health insurance, post-acute health service utilization is also associated with functional outcomes after injury. The relationship between health service utilization and health outcomes is complex. Patients who are more severely injured or have more health problems should be expected to use more health services. As a result, some studies report a negative association between increased health service utilization and health outcomes (Guilcher et al., 2010; Davidson et al., 2011). However, other 
studies have indicated that increased access to healthcare providers is associated with improvements in functional outcome (Castillo, MacKenzie, Archer, Bosse, \& Webb, 2008; Kucan et al., 2010). Castillo et al (2008) reported that patients with an unmet need for physical therapy were significantly less likely to experience improvements in physical impairment.

Similar to research on insurance status and outcome, studies that have examined the effect of health service utilization on outcome after injury have primarily looked at pediatric and elderly patients or patients affected by neurological injury. In the pediatric population, a shortened length of stay in a rehabilitation facility was not associated with reduced functional outcome (FIM), however, fewer children returned to a similar level of community participation, as measured by return to school (Rice et al., 2004). In Medicare patients with hip fractures, it was found that those who were discharged to home health care, rehabilitation facilities, or nursing homes achieved better functional outcomes oneyear post injury than those who were discharged home (Kane et al., 1998). Medicare patients discharged to home health care or rehabilitation facilities achieved the greater improvement in function compared to patients sent to nursing homes (Kane, Chen, Blewett, \& Sangl, 1996; Kane et al.,1998; Kane et al., 2000).

Patients with neurological injuries that receive post-acute care have also been found to attain higher functional status. Andelic et al. reported that patients who received early onset and continuous rehabilitation achieved better functional outcomes that those who did not (2012). Among traumatic brain injury patients, receipt of neuropsychological evaluation is also associated with achieving a higher level of functional independence. Both private and publicly insured patients were equally likely to receive neuropsychological evaluations (Schatz, Hughes, \& Chute, 2001).

Loss to follow-up and fragmentation of the U.S. healthcare system makes obtaining data on both health outcomes and medical care use after injury difficult. Loss to follow-up can be a problem for all injury researchers as patients often seek follow-up care from providers who did not provide acute care for injuries. However, even in cases where follow-up appointments are made with the acute care provider, failure to return for care occurs. Not all patients are equally likely to receive follow-up care after injury and patient characteristics such as race and income are associated with an increased risk of failure to follow-up (Leukhardt et al., 2010). Groups that have difficulty accessing health services after injury such as the uninsured are often unable to have their healthcare needs met and, in turn, likely suffer worse outcomes (Brown 2010; Derett et al., 2012). One major determinant of access to health services is insurance coverage, an important predictor of medical care use after injury.

\section{Effects of Insurance Status on Medical Care Use after Injury}

Insurance status greatly influences medical care use in the injured population.

Researchers have found that medical care use varies according to insurance status across 
all phases of care including treatment at the time of injury, utilization of rehabilitative services, and follow-up care provided in the ambulatory care setting.

Studies have found that insurance status influences whether or not an individual will receive treatment at the time of injury. Individuals with non-emergent injuries make decisions on when and whether or not to pursue medical treatment based on insurance status and coverage. Berdahl et al (2010) reported that the odds of seeking treatment for a work-related injury was 33\% lower for uninsured workers compared to those with private insurance. Disparities in access to health services may partially explain the observed differences in treatment seeking behaviors between the uninsured and insured.

In addition to treatment seeking differences, a patient's insurance status may lower health care providers' propensity to provide care. Studies in the pediatric population have found that children with Medicaid, no insurance, or receiving charity care are more likely to experience a delay in care for their injuries and visit multiple hospitals before being treated definitively (Sabharwal, Zhao, McClemens, \& Kaufmann, 2007). Similar to findings in the pediatric population, uninsured adults with femur fractures are more likely to be transferred to another hospital even after controlling for confounders (Archdeacon, Simon, \& Wyrick, 2007). Obstacles in receiving care may result in delays in treatment, under-treated injuries, and unnecessary complications that result in poor outcomes for uninsured patients.

Insurance status is also a determining factor in the quantity and scope of services provided to injured patients. In the traumatic injury population, studies have found that uninsured patients undergo fewer operative procedures, spend less time in the intensive care unit, have shorter and less costly hospital stays, and receive fewer physical therapy sessions as inpatients (Haas \& Goldman, 1994; Doyle 2005; Alban et al., 2010; Taghavi et al., 2012). Among injured patients who visit the ED, uninsured individuals are less likely to be admitted to the hospital after adjusting for clinical factors (Selassie, Pickelsimer, Frazier, \& Ferguson, 2004). Uninsured ED patients also receive significantly fewer radiographic studies (White, French, Zwemer, \& Fairbanks, 2007). These findings indicate that there is a general trend for providers to minimize resource expenditures on uninsured patients. As a result, the intensity and quality of health services provided to injured patients may vary according to insurance status.

Several studies have also focused on disparities in access to post-acute care and differences in hospital discharge locations after major injury for the uninsured. Compared to insured patients, uninsured patients are less likely to be discharged to home health care, rehabilitation, and skilled nursing facilities (Osberg \& Unsworth, 1997; Claridge et al. 2006; Englum et al., 2011; Nirula, Nirula, \& Gentilello, 2009; Farrell et al., 2010; Sacks, Hill, \& Rogers, 2011; Heffernan et al. 2011; Taghavi et al., 2012). Other studies have found that patients without insurance report that they did not receive an adequate number of visits to outpatient providers of rehabilitation services (McCarthy, Ewashko, $\&$ MacKenzie, 1998). Studies have reported conflicting findings on the effect of public insurance on receipt of post-acute care. After controlling for physiological and sociodemographic variables, studies have found that publicly insured patients are more 
likely to be discharged to rehabilitation and nursing facilities than privately insured patients (Englum et al., 2011). However, other studies have found that publicly insured patients have an increased likelihood of being discharged to certain types of post-acute care facilities depending on whether they are Medicare or Medicaid recipients. Sacks et al found that both Medicaid and Medicare patients are less likely to be discharged home than commercially insured patients and are more likely to be sent to a skilled nursing facility (Sacks et al., 2011). However, Medicare patients but not Medicaid patients are more likely to enter inpatient rehabilitation after injury. Other studies have noted that all publicly insured patients have a higher likelihood of being discharged to an inpatient rehabilitation or nursing facility, but there are no differences in discharge to home health care compared to privately insured patients (Englum et al., 2011; Lim, Hoffmann, \& Brasel, 2007; Chan et al., 2001). Overall these findings suggest that uninsured patients have decreased access to rehabilitation services that would improve their chances of reaching full recovery. The effect of public insurance on the receipt of rehabilitation services in less conclusive.

Insurance status also influences the likelihood of patients receiving follow-up care with ambulatory care providers (McCarthy et al., 1998; Slomine et al., 2006). Hadley et al. (2007) reported that uninsured individuals who sought medical treatment for their injuries were as likely as insured individuals to receive a recommendation for follow-up care. However, uninsured participants were significantly more likely to receive no follow-up care and significantly less likely to receive all of the recommended follow up care. Patients in the uninsured group were also more likely to stop treatment prior to reaching full recovery. Overall this study found that uninsured patients had significantly fewer office-based visits and prescription medicines than their insured counterparts (Hadley, 2007). In another study examining perceived need and use of physical therapy services after injury, Castillo et al (2005) reported that uninsured patients were more likely to perceive a need for physical therapy, but less likely to receive those services.

Insurance coverage is strongly associated with the amount and quality of medical care individuals receive after injury. The findings from this review suggest that both providers and patients share agency for disparities in obtaining injury-related healthcare. Providers tend to reduce medical care use by minimizing resource expenditure on uninsured patients; whereas, uninsured patients are less likely to seek treatment and follow-up care for their injuries. However, disparities in access to health services may explain the observed differences in treatment seeking behaviors between the insured and uninsured.

\section{Review of Methodology Literature}

This dissertation primarily utilized two quantitative approaches to analyze longitudinal outcomes in the injured population. First, it examined health trajectories of the injured population using latent class growth analysis, also termed group-based trajectory modeling (GBTM). The purpose of this modeling technique is to estimate distinct growth curves of latent classes within a heterogeneous population. This method 
also estimates the probability of belonging to a specific class. Second, this study performed a mediation analysis using the causal steps approach popularized by Baron and Kenny (1986). In mediation analysis, the independent variable of interest is hypothesized to impact the dependent variable through a third intervening variable. This technique allows researchers to quantify the direct and indirect effects of an independent variable in order to provide more information on the causal process by which the independent variable affects the dependent variable.

\section{Group-based Trajectory Modeling in Injury Research}

The use of trajectory analysis has most commonly been applied in the social sciences; however, its popularity in health outcomes research has been increasing. Several longitudinal studies examined in this review have used this approach to analyze outcomes after injury. Soberg et al (2012) studied long-term physical and mental health trajectories in a cohort of trauma patients. Their findings indicated that the recovery trajectory for physical and mental health differ and that both personal and injury characteristics were predictive of outcomes. Other studies have investigated the impact of injury on long-term healthcare use. Both Koehoorn et al (2008) and Collie and Prang (2013) identified distinct trajectories of health service utilization in the occupational injury and traumatic brain injury populations using group-based trajectory models. Lastly, trajectory analysis has been applied to injury research in order to examine probabilities of being injured over time. Koehoorn et al (2010) identified four distinct trajectories predicting the probability of back injury in workers in heavy industries. In summary, group-based trajectory modeling has not been used extensively in injury research; however, it has been applied in diverse ways.

\section{Mediation Analysis in Health Services Research}

Similar to group-based trajectory modeling, mediation analysis is another analytical method more commonly used in the social sciences. Over the last five years, several studies investigating mediators between health service utilization and health outcomes have been published, although none have specifically studied the injured population. Most of these studies attempt to explain why certain groups of people tend to have worse health outcomes. One study investigating the role of health service utilization in health disparities found that the ability to afford medication mediates the relationship between race and poor health outcomes (Cobaugh et al 2008). Another study found that Medicaid acts as a suppressor of racial disparities in health for children with special needs (Rose et al 2010). Gorey et al (2012) investigated the relationship between poverty, health insurance coverage, and quality of care received in patients with colon cancer. They reported that insurance mediates the quality of cancer care received and that living in poor neighborhoods moderates this effect. None of the studies examined in this review used mediation analysis to determine the effect of insurance status on outcome after injury. Although at present mediation analysis is less commonly used in health 
services research, it has the potential to provide richer information than traditional regression analyses.

\section{Gaps in the Literature}

There is limited research on long-term changes in health status after injury, particularly in the United States. Barriers to investigating long-term outcomes of injury include difficulties such as obtaining follow-up assessments with patients over time, acquiring unbiased pre-injury baseline measurements, and accurately assessing receipt of post-acute care services due to fragmentation of the healthcare system. Furthermore, there have been challenges in obtaining a nonselective "all injury" population, and studies have primarily focused on conducting research in injury-specific populations (e.g. spinal cord injury). Most studies published on health outcomes after injury follow patients for only 6, 12, or 24 months, although some European studies have looked at functional status for up to 10 years in small populations (Polinder 2010; Andelic et al., 2009).

This study attempted to address some of the barriers former studies have faced. By using data from the Health and Retirement Study, 5 follow-up assessments and up to 12 years of post-injury data will be available. This database also allows for the identification of people who are injured during the study so that pre-injury baseline measurements will be free from recall bias. Lastly, measurements of medical care use are provided by study participants. Self-reported utilization allows for the inclusion of individuals without insurance coverage, who would otherwise be missed if the utilization measurement relied on claims data. Self-reported utilization may give a more complete picture of services used since it includes services that are paid for out of pocket or covered by a secondary form of insurance.

\section{Significance of Proposed Study}

Studying long-term injury outcomes in the U.S. population will shed light on the recovery process, identify common functional deficits that persist years after injury, and determine which of the 30 million Americans injured every year will go on to experience functional limitations. Longitudinal studies, such as the one proposed, allow for different inferences to be made from data. In this study, the aim is to gain improved understanding about the recovery process so that patients at-risk for poor long-term outcomes can achieve their optimal post-injury outcome. By also examining the mediating effect of medical care use, we can determine if medical care use has a sustained, systematic effect on the recovery process over time. Studying the role of the relationship between insurance status and medical care use on health outcomes will provide additional information about how these modifiable factors shape the recovery process. Elucidating the mechanism of health insurance's effect on post-injury functional status is clinically relevant and findings can be applied to initiatives concerning discharge planning, determining the adequate amount and duration of rehabilitation services, as well as 
assessing pain management benchmarks. Results from this study could be used to support the integration of acute and post-acute services into a "seamless system of care" for injured patients and also guide reform of health insurance policies. 


\section{CHAPTER 3. METHODS}

\section{Objective of the Study}

The objective of this study is to model functional status trajectory after injury and to determine how insurance status and medical care use work together to define longterm functional outcomes. Studies have extensively documented that insurance status is one of the strongest predictors of outcome after injury, yet the causal mechanism by which insurance exerts its effect is not fully described. This study is divided into two parts (1) identifying injury subgroups at-risk for enduring post-injury functional limitations and their associated trajectories of recovery and dysfunction and (2) assessing the impact of health insurance on medical care use that influences long-term changes in functional status.

\section{Data Source}

Data from the Health and Retirement Study (HRS) was used for the study. The HRS is a longitudinal panel study that surveys a representative sample of more than 26,000 Americans over the age of 50 every two years. Data is collected on health transitions that individuals undergo towards the end of their working years and during retirement. Data from the HRS spans nearly twenty years and contains detailed information on income, work, assets, pension plans, health insurance, disability, physical health and functioning. Six waves of data, collected from 1998 to 2008, were used for this study.

Datasets were created from HRS Core RAND Enhanced Fat files and the RAND Income and Wealth Imputation files from years 1998, 2000, 2002, 2004, 2006, and 2008. The RAND files differ from the Public Release HRS files in that they have a simplified data structure that has a single observation for each respondent that includes householdlevel variables. These files were also created to improve the process of merging multiple years of data together for longitudinal analyses. Full documentation on all HRS data files is publicly available and can be found on the University of Michigan's Health and Retirement Study website (http://hrsonline.isr.umich.edu/).

\section{Study Sample}

Data from the HRS Core Survey was used to determine eligibility for this study. Participants were selected based on the following criteria: (1) participant completed surveys in 1998 and 2000, (2) participant indicates that they suffered a recent accident or injury in the 2000 survey, (3) participants indicate that they did not suffer an injury in the 1998 survey, and (4) participant completes at least one post-injury survey after 2000. Injured participants were identified based on responses to the following items from the 2000 HRS Core Survey (Table 3-1). 
Table 3-1. 2000 HRS Core Survey Injury Variables

\begin{tabular}{|c|c|c|c|}
\hline $\begin{array}{c}\text { HRS } \\
\text { Survey } \\
\text { Variable }\end{array}$ & HRS Survey Label & $\begin{array}{c}\text { No. of } \\
\text { Responses }\end{array}$ & Included if Response = \\
\hline G1345 & B12B. INJURE & 1 & Yes \\
\hline G1348 & B13. BROKEN HIP & 1 & Yes \\
\hline G1377M1M & $\begin{array}{l}\text { B19A-1. OTHER } \\
\text { HEALTH SPEC- } \\
\text { MENT } 1 \text { - MASKE }\end{array}$ & 194 & $\begin{array}{l}\text { Injuries and traumas: broken bones; } \\
\text { pulled muscles; strains; tendon damage; } \\
\text { burns, lacerations; concussion; side } \\
\text { effects/conditions due to surgery }\end{array}$ \\
\hline G1377M2M & $\begin{array}{l}\text { B19A-2. OTHER } \\
\text { HEALTH SPEC- } \\
\text { MENT } 2 \text { - MASKE }\end{array}$ & 194 & $\begin{array}{l}\text { Injuries and traumas: broken bones; } \\
\text { pulled muscles; strains; tendon damage; } \\
\text { burns, lacerations; concussion; side } \\
\text { effects/conditions due to surgery }\end{array}$ \\
\hline G4308M1M & $\begin{array}{l}\text { GD1A-1.CAUSE } \\
\text { PROB - MASKED }\end{array}$ & 194 & $\begin{array}{l}\text { Injuries and traumas: broken bones; } \\
\text { pulled muscles; strains; tendon damage; } \\
\text { burns, lacerations; concussion; side } \\
\text { effects/conditions due to surgery }\end{array}$ \\
\hline G4308M2M & $\begin{array}{l}\text { GD1A-2.CAUSE } \\
\text { PROB - MASKED }\end{array}$ & 194 & $\begin{array}{l}\text { Injuries and traumas: broken bones; } \\
\text { pulled muscles; strains; tendon damage; } \\
\text { burns, lacerations; concussion; side } \\
\text { effects/conditions due to surgery }\end{array}$ \\
\hline G4331 & $\begin{array}{l}\text { GD9. RESULT OF } \\
\text { ACCIDENT }\end{array}$ & 1 & Yes \\
\hline G4534 & $\begin{array}{l}\text { GD131.INJ AT } \\
\text { WORK }\end{array}$ & 1 & Yes \\
\hline G4554M1M & $\begin{array}{l}\text { GJ1A-1.CAUSE } \\
\text { PROB - MASKED }\end{array}$ & 194 & $\begin{array}{l}\text { Injuries and traumas: broken bones; } \\
\text { pulled muscles; strains; tendon damage; } \\
\text { burns, lacerations; concussion; side } \\
\text { effects/conditions due to surgery }\end{array}$ \\
\hline G4554M2M & $\begin{array}{l}\text { GJ1A-2.CAUSE } \\
\text { PROB - MASKED }\end{array}$ & 194 & $\begin{array}{l}\text { Injuries and traumas: broken bones; } \\
\text { pulled muscles; strains; tendon damage; } \\
\text { burns, lacerations; concussion; side } \\
\text { effects/conditions due to surgery }\end{array}$ \\
\hline G4559M1M & $\begin{array}{l}\text { GJ1F-1.CAUSE } \\
\text { PROB - MASKED }\end{array}$ & 194 & $\begin{array}{l}\text { Injuries and traumas: broken bones; } \\
\text { pulled muscles; strains; tendon damage; } \\
\text { burns, lacerations; concussion; side } \\
\text { effects/conditions due to surgery }\end{array}$ \\
\hline G4559M2M & $\begin{array}{l}\text { GJ1F-2.CAUSE } \\
\text { PROB - MASKED }\end{array}$ & 194 & $\begin{array}{l}\text { Injuries and traumas: broken bones; } \\
\text { pulled muscles; strains; tendon damage; } \\
\text { burns, lacerations; concussion; side } \\
\text { effects/conditions due to surgery }\end{array}$ \\
\hline G4576 & $\begin{array}{l}\text { GJ8.RESULT OF } \\
\text { ACCIDENT }\end{array}$ & 1 & Yes \\
\hline G4598 & $\begin{array}{l}\text { GJ16.RESULT OF } \\
\text { ACCIDENT }\end{array}$ & 1 & Yes \\
\hline G4625 & $\begin{array}{l}\text { GJ27. RESULT OF } \\
\text { ACCIDENT }\end{array}$ & 1 & Yes \\
\hline
\end{tabular}


Table 3-1. Continued

\begin{tabular}{|c|c|c|c|}
\hline $\begin{array}{c}\text { HRS } \\
\text { Survey } \\
\text { Variable }\end{array}$ & HRS Survey Label & $\begin{array}{c}\text { No. of } \\
\text { Responses }\end{array}$ & Included if Response $=$ \\
\hline G4630M1M & $\begin{array}{l}\text { GJ29A-1.CAUSE } \\
\text { PROB - MASKED }\end{array}$ & 194 & $\begin{array}{l}\text { Injuries and traumas: broken bones; } \\
\text { pulled muscles; strains; tendon damage; } \\
\text { burns, lacerations; concussion; side } \\
\text { effects/conditions due to surgery }\end{array}$ \\
\hline G4630M2M & $\begin{array}{l}\text { GJ29A-2.CAUSE } \\
\text { PROB - MASKED }\end{array}$ & 194 & $\begin{array}{l}\text { Injuries and traumas: broken bones; } \\
\text { pulled muscles; strains; tendon damage; } \\
\text { burns, lacerations; concussion; side } \\
\text { effects/conditions due to surgery }\end{array}$ \\
\hline G4648 & $\begin{array}{l}\text { GJ30.RESULT OF } \\
\text { ACCIDENT }\end{array}$ & 1 & Yes \\
\hline G4941 & $\begin{array}{l}\text { GJ131.INJURED AT } \\
\text { WORK }\end{array}$ & 1 & Yes \\
\hline
\end{tabular}




\section{Measures}

\section{Outcome Variable}

Functional Status is the primary outcome of interest in this study. Specifically, a scaled variable corresponding to participants' functional limitations was generated for the analysis. Five items from the RAND HRS data were incorporated into the scaled variable. One item pertained to difficulty performing usual activities due to pain, which allowed for a binary response $(0=$ pain does not cause difficulty, $1=$ pain does cause difficulty). The four other items included in the scale were indices for functional limitations created by RAND based on responses to questions about activities of daily living. These included the mobility index, large muscle index, gross motor skills index, and fine motor skills index. All of the RAND indices are the sum of the number of difficulties a participant has in performing a specific set of tasks relating to each index. The tasks included were walking several blocks, walking one block, walking across the room, climbing several flights of stairs, climbing one flight of stairs, sitting for two hours, getting up from a chair, stooping or kneeling or crouching, pushing or pulling a large object, bathing, picking up a dime, eating, and dressing. RANDS selected these tasks to make up the functional limitations indices because of their consistency across waves.

In order to create the functional limitations scale, a factor analysis was conducted. The analysis first examined whether the five items had a single factor in common for each wave of data. After confirming there was only one factor based on the initial eigenvalues, a reliability analysis was conducted. The Cronbach's Alphas for each item being included in the scale and also deleted from the scale were computed. The Alpha values were then used to determine whether any items needed to be omitted from the scale. Lastly, the functional limitations scale was computed by averaging all five items together with each having an equal weight.

\section{Independent Variables}

Health Status Indicators included in the model are number of health conditions and recent injury. The total number of health conditions is the sum of indicators for whether or not a physician ever told the respondent that they have any of the following diseases: high blood pressure, heart disease, stroke, diabetes, cancer, lung disease, psychiatric problems, and arthritis.

Injury Status was a dichotomous variable indicating whether or not the respondent had been injured since the previous wave.

Insurance Status was categorized based on the respondent's primary type of insurance, $0=$ no insurance, $1=$ public insurance (specifically Medicare and Medicaid), and $3=$ private insurance. 
Socioeconomic Status Indicators include household assets, educational attainment, and work status. Assets include the total amount of checking and savings accounts, retirement accounts, investments, and trusts. Household assets rather than income was selected as a socioeconomic status indicator because it was more stable since most individuals in the sample retire during the study. Educational attainment was categorized into less than high school, high school graduate, some college, and college graduate. Work status will be categorized as currently working or not working.

Demographic Covariates included in the model are age, gender, race/ethnicity, and social support. Because all participants in the study are older adults, age was treated as a categorical variable in order to make more meaningful comparisons between the elderly. Age was categorized as 55 and younger, 56-60, 61-65, 66-70, 71 and older. Race/ethnicity was categorized as non-Hispanic white, black, Asian, and Hispanic. Social support was a dichotomous variable that indicated whether or not the respondent lived with a spouse.

\section{Mediating Variables}

Medical Care Use Indicators that were included as mediators in the model include outpatient doctor visits, prescription and outpatient surgery. Outpatient doctor visits was measured as the number of times the respondent has visited the doctor in the past two years. Prescription drug usage was a dichotomous variable that indicated whether the respondent regularly takes prescription medication $(0=$ does not use prescription drugs, $1=$ uses prescription drugs). Outpatient surgery was specified as a dichotomous variable indicating whether or not the respondent has had outpatient surgery in the last two years $(0=$ no surgery, $1=$ surgery $)$.

\section{Analyses}

This study is a retrospective, longitudinal cohort study that investigates its aims primarily through the utilization of descriptive statistics, trajectory analysis, regression analysis, and mediation analysis. In short, Part I of this study employs a person-centered analytical approach that focuses on the relationship between individuals with the goal of classifying them into subgroups. Part II of the study is a variable-centered analytical approach that studies the relationship among variables with the goal of describing how independent and outcome variables are related by way of a mediating variable.

A list of all variables used in analyses is shown in Table 3-2.

Descriptive statistics of the demographic, health status, socioeconomic, medical care use, and functional status variables were calculated including means, standard deviations, and frequencies. Associations between the individual factors, medical care use and functional status were determined using $\chi^{2}$ tests and tests. All tests were two tailed tests with $\alpha=.05$. 
Table 3-2. List of Variables for Statistical Models

\begin{tabular}{|c|c|c|}
\hline ID & Variable & Description of Variable \\
\hline \multicolumn{3}{|r|}{ Dependent Variables } \\
\hline 1 & MOBIL & Mobility Index (score range from 0 - 5 ) \\
\hline 2 & LGMUS & Large Muscle Index (score range from 0 - 4) \\
\hline 3 & FINE & Fine Motor Skills Index (score range from 0 - 3) \\
\hline 4 & GROSS & Gross Motor Skills Index (score range from 0 - 4) \\
\hline 5 & $\begin{array}{l}\text { PAIN } \\
\text { FUNCSTA }\end{array}$ & $\begin{array}{l}\text { Difficulty performing activities due to pain }(0=\text { pain does not } \\
\text { cause difficulty; } 1=\text { pain does cause difficulty) }\end{array}$ \\
\hline 6 & $\mathrm{~T}$ & Functional limitation scale (0 - 100) \\
\hline \multicolumn{3}{|r|}{ Literature-Based Covariates } \\
\hline 7 & AGE & $\begin{array}{l}\text { Age Category }(0=55 \text { and younger; } 1=56-60 ; 2=61-65 ; 3= \\
66-70 ; 4=71 \text { and older }\end{array}$ \\
\hline 8 & GEN & Gender $(0=$ male; $1=$ female $)$ \\
\hline 9 & RACE & $\begin{array}{l}\text { Race/Ethnicity }(0=\text { non-Hispanic white; } 1=\text { non-Hispanic } \\
\text { black; } 2=\text { Hispanic; } 3=\text { other })\end{array}$ \\
\hline 10 & EDU & $\begin{array}{l}\text { Education }(0=\text { less than high school; } 1=\text { high school diploma; } 2 \\
=\text { some college; } 3=\text { bachelor's degree or higher })\end{array}$ \\
\hline 11 & COUPLE & $\begin{array}{l}\text { Social Support }(0=\text { does not live in coupled household; } 1=\text { lives } \\
\text { in coupled household })\end{array}$ \\
\hline \multicolumn{3}{|r|}{ Health Status Variables } \\
\hline 12 & INJURY & $\begin{array}{l}\text { Recent Injury ( } 0=\text { not injured since previous wave; } 1=\text { injured } \\
\text { since previous wave) } \\
\text { Number of health conditions (count of comorbidities reported at } \\
\text { each wave including high blood pressure, heart disease, stroke, } \\
\text { diabetes, cancer, lung disease, psychiatric problems, and } \\
\text { arthritis) }\end{array}$ \\
\hline \multicolumn{3}{|r|}{ Socioeconomic Status Variables } \\
\hline 14 & ASSET & Household Assets \\
\hline 15 & WORK & Work Status $(0=$ not working; $1=$ working $)$ \\
\hline \multicolumn{3}{|r|}{ Insurance Status Variables } \\
\hline 16 & INSTYPE & Primary Type $(0=$ Private; $1=$ Public; $2=$ No Insurance $)$ \\
\hline \multicolumn{3}{|r|}{ Medical Care Use Variables } \\
\hline 17 & DRVISIT & Number of Visits to Physicians (count of visits) \\
\hline 18 & $\mathrm{RX}$ & Prescriptions $(0=$ does not use $\mathrm{Rx} ; 1=$ uses $\mathrm{Rx})$ \\
\hline 19 & OPTSURG & $\begin{array}{l}\text { Outpatient Surgery }(0=\text { did not have surgery; } 1=\text { did have } \\
\text { surgery) }\end{array}$ \\
\hline \multicolumn{3}{|r|}{ Identification Variable } \\
\hline 20 & SUBJID & Participant's ID Number \\
\hline
\end{tabular}




\section{Part I. Classification of Functional Status Trajectory after Injury and Identification of Predictors of Long-term Functional Outcomes}

Group-Based Trajectory Analysis. The first aim of this study is to identify classes of individuals within the injured population that have varying recovery patterns, or functional status trajectories. Outcome trajectories for functional status were determined by fitting a group-based trajectory model (GBTM) to the data. GBTM is a semi-parametric statistical technique that helps to identify homogeneous subpopulations within a heterogeneous population. This procedure is used when the data follows a pattern of change in which both the strength and the direction of the relationship between the independent and dependent variables differ between cases. This purpose of this type of analysis is to identify distinct subgroups of individuals that follow a similar pattern of change over time on a variable of interest that were previously unknown. For this study, subgroups within the injured population that follow a similar post-injury functional status trajectory were identified. The outcome variable used in the GBTM analysis was the functional limitation scale.

Rationale for Model Selection. The rationale for selecting this modeling technique for part I of the study is that it is able to describe a dynamic outcome as a singular, longitudinal trend for each subgroup within a population. GBTM is largely exploratory and this modelling approach was utilized by the current study to provide essentially descriptive information about participants' long-term outcomes. Furthermore, the findings of this analytical approach lend itself to visual presentation and can be easily summarized graphically to improve understanding among policy-makers or non-technical audiences (Jones \& Nagin 2007). Additionally, this approach is person-centered, meaning that it aims to describe relationships between individuals rather than relationships between variables. Person-centered analyses provide information that is not readily obtained from variable-centered analyses by classifying participants into distinct groups based on their outcomes. This feature of GBTM is particularly relevant for researchers interested in investigating patient-centered outcomes. Therefore, this method provides information on easily identifiable trends in the data and can identify meaningfully distinct subpopulations.

Conducting Trajectory Analysis. The first step of conducting GBTM is to determine a priori the maximum number of trajectory subgroups. Based on Iwashyna's descriptions of trajectories of recovery and dysfunction after acute illness, this study assumes there are potentially five distinct functional trajectories after injury (Iwashyna, 2012). These subgroups include the following trajectories:

- Trajectory 1: Static, High Functioning - This group is characterized as having a consistently low score on the functional limitations scale.

- Trajectory 2: Dynamic, Big Hit- This group is characterized as having an initial increase in functional limitations after injury followed by a gradual recovery over time (i.e. decreasing functional limitations score). These individuals experience significant improvement, but may not return to their pre-injury functional status.

- Trajectory 3: Dynamic, Slow Burn - This group is characterized as having an 
initial increase in functional limitations after injury followed by a further decline in functioning over time (i.e. increasing functional limitations score).

- Trajectory 4: Dynamic, Relapsing Recurrence - This group is characterized as having fluctuating scores on the functional limitations scale. Because higher and lower scores at each time point average each other out, this trajectory may manifest itself as a stable trend over time with an intermediate functional limitations score.

- Trajectory 5: Static, Low Functioning - This group is characterized as having a consistently high score on the functional limitations scale.

After identifying a theoretical maximum number of subgroups, the optimum number of subgroups for the data was determined by examining Bayesian Information Criterion (BIC) values for successive models that increased the number of subgroups by one, beginning with a one group model. All group orders in each model were set as quadratic during this step. The differences in BIC values were then used to calculate Bayes factors using the following formula

$$
\text { Bayes Factor }=\mathrm{e}^{\mathrm{BICi}-\mathrm{BICj}}
$$

where $\mathrm{BIC}_{\mathrm{i}}$ is the $\mathrm{BIC}$ value for the more complex model and $\mathrm{BIC}_{\mathrm{j}}$ is the $\mathrm{BIC}$ value for the simpler model. The Bayes Factors were interpreted using Jeffrey's Scale of Evidence and the model with the number of subgroups that best fit the data was selected (Nagin, 1999). Once the optimum number of subgroups was determined for the data, models comparing various trajectory shapes were run. The orders for each trajectory were determined based on BIC values and substantive knowledge of the data. Analyses were unconditional during this step, meaning that the outcome variable was the lone variable included in the model. This allows for the outcome to be modeled without the influence of other variables.

Once the number and shape of each trajectory was determined, time-varying covariates were added to the model. The variable injury status was incorporated into the model to account for changes in functional limitations due to recent injury. Time-stable covariates, or risk factors, were not incorporated into this step of the trajectory analysis because it is followed by more in-depth regression analyses investigating variables that can predict and discriminate between subgroups. Time-stable covariates do not influence the observed trajectories produced by the model, however, these covariates are used by the model to predict subgroup membership. Because follow-up logistic and multinomial regression analyses were used to identify baseline subgroup predictors, they were omitted from the group-based trajectory model.

All GBTM analyses were conducted using the TRAJ procedure that employed a censored normal model in SAS Version 9.3 (Cary, NC). The censored normal model was selected as it is appropriate for scaled data that tends to cluster at minimum and maximum values on the scale. Because PROC TRAJ does not have a closed form solution for maximum likelihood estimates, it is susceptible to producing local solutions. In order to protect from this, start values for parameter estimates were adjusted during the 
modeling process.

After the previous modeling steps were complete, $95 \%$ confidence intervals for each trajectory were calculated in order to determine which groups differ from one other at each time point. Differences in coefficients and constant terms of trajectories were examined using the SAS macro trajtest, which conducts the Wald test for testing significance of predictors. Baseline demographic, socioeconomic, health status, and insurance characteristics for each trajectory are also reported. Lastly, characteristics of changes in insurance status and medical care use over time were examined for each trajectory.

Assessing Predictors of Functional Status Trajectories. After trajectory classes were determined, multivariate regression analysis was used to identify significant predictors for each class. Study participants were assigned to the trajectory to which they had the highest probability of belonging to through the use of posterior probabilities. This step is automatically carried out during the TRAJ procedure and the group membership for each observation is included in an output dataset that was merged with the original dataset.

Bivariate Logistic Regression Analyses. After being assigned to a trajectory, logistic regression was used to determine predictors that can discriminate between trajectories that are most distinct, such as groups that show significant improvement versus significant decline in functional status over time. Information from this analysis can be used to compare two subgroups that have similar values early on in the study but then diverge over time. Therefore, these predictors can be used to determine variables that drive improvement or decline. All trajectories were compared to the trajectory described as the hypothesized "Big Hit" recovery pattern, in order to identify predictors that cause patients to recover in a way that differs from what is clinically expected. Additionally, two models comparing trajectories with high and intermediate functional limitations were also conducted. Although the analysis identified five trajectories, one of the trajectories differed from the hypothesized subgroup. Instead of a "Relapsing Recurrence" trajectory, the analysis identified a "Long-term Improvement" trajectory. Because of the small number of observations in the "Static - High Functioning" and "Static - Low Functioning" trajectories, it was not possible to statistically test for predictors between these two groups. A list of models ran is included below and the second trajectory listed was used as the reference group. In other words, the probability that an individual will belong to the first trajectory subgroup listed compared to the second subgroup is being modeled. Only individuals belonging to the subgroups being compared were included in the bivariate logistic regression models.

- Model 1: Trajectory 1: Static, High Functioning vs. Trajectory 2: Dynamic, Big Hit

- Model 2: Trajectory 3: Dynamic, Slow Burn vs. Trajectory 2: Dynamic, Big Hit

- Model 3: Trajectory 4: Dynamic, Long-term Improvement vs. Trajectory 2: Dynamic, Big Hit 
- Model 4: Trajectory 5: Static, Low Functioning vs. Trajectory 2: Dynamic, Big Hit

- Model 5: Trajectory 4: Dynamic, Long-term Improvement vs. Trajectory 3: Dynamic, Slow Burn

- Model 5: Trajectory 4: Dynamic, Long-term Improvement vs. Trajectory 5: Static, Low Functioning

Multinomial Logistic Regression Analyses. Multinomial regression was also conducted to identify significant predictors of each of the functional status trajectory subgroups. This analysis provides information about predictors of each subgroup while using the entire sample. For example, certain variables may be strongly associated with a trajectory that has consistently high functional status values compared to another subgroup. The reference group used in the multinomial regression analysis was the "Big Hit" trajectory.

The rationale for using both types of regression analysis is that logistic regression will identify predictors that discriminate between two specific subgroups and multinomial regression will identify predictors that are associated with each type of trajectory.

Regression analyses were carried out in SAS using PROC LOGISITC. Model covariates for both bivariate and multinomial logistic regression included baseline age, gender, race/ethnicity, education, coupled household status, household asset quintile, work status, number of health conditions, and insurance status. Odds ratios and 95\% confidence intervals for each model are reported.

\section{Part II. Assessing the Role of the Relationship between Health Insurance and Medical Care Use on Long-term Functional Outcomes}

Identifying Mediators of Insurance Status. For Part II of the study, the objective was to assess the mechanism by which health insurance status goes on to affect functional status after injury. Specifically, the aim of this section of the study was to determine whether or not medical care use acts as a mediator between respondents' insurance status and their functional outcome.

Overview of Mediation Analysis. Based on the conceptual framework, this study proposes that insurance status indirectly affects functional outcomes through medical care use. This model can test whether the indirect effect is either completely or partially responsible for the relationship between insurance status and health service utilization. Given that insurance status is a strong and consistent predictor of outcomes in the injured population, it is important to assess its relationship to functional status in a way that can provide information that is more than simply predictive.

The diagram in Figure 3-1 depicts a simple mediation model where $\mathrm{X}$ represents the independent variable, $\mathrm{M}$ is a mediating variable, and $\mathrm{Y}$ is the outcome variable. This model assumes that a significant proportion of the effect of $\mathrm{X}$ on $\mathrm{Y}$ is actually due to the 
Without Mediation

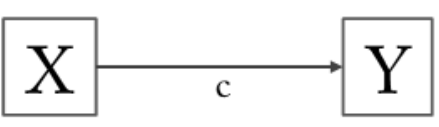

With Mediation

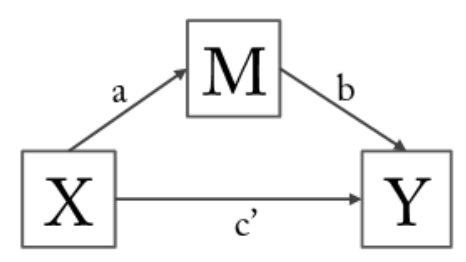

Figure 3-1. Simple Mediational Model 
effect of $\mathrm{X}$ on $\mathrm{M}$ and, in turn, $\mathrm{M}$ on $\mathrm{Y}$. By comparing the direct effects of $\mathrm{X}$ on $\mathrm{Y}$ without $\mathrm{M}$ and the direct effect of $\mathrm{X}$ on $\mathrm{Y}$ with $\mathrm{M}$, information about the mechanism by which $\mathrm{X}$ influences $\mathrm{Y}$ can be gleaned. Essentially this model is being used in an attempt to explain how $\mathrm{X}$ exerts its effect on $\mathrm{Y}$.

In the model, $c$ refers to the total effect of $\mathrm{X}$ on $\mathrm{Y}$ and $c$ ' is described as the direct effect of X on Y. Additionally, the coefficient of $a b$ is referred to as the indirect effect or mediated effect. The total effect is the amount in which two individuals with a one unit difference in $\mathrm{X}$ are estimated to differ on $\mathrm{Y}$ through both direct and indirect pathways. The direct effect is the effect of $\mathrm{X}$ on $\mathrm{Y}$, independent of $\mathrm{X}$ 's effect on $\mathrm{M}$. The indirect effect is the effect on Y of X's influence on M which in turn affects Y. The paths $c, c^{\prime}, a$, and $b$ can be estimated using a series of regressions described as the causal steps approach by Baron and Kenny (1986).

Mediation Model Path Diagram. Based on the conceptual model proposed by this study, the figure below depicts the relationships between measured variables in this study and their corresponding regression coefficients (Figure 3-2). These coefficients are used to calculate the total, direct, and indirect effects of insurance status on functional limitations. The model is characterized by having a single multicategorical independent variable, insurance status $(\mathrm{X})$, and three mediating variables, doctor visits $\left(\mathrm{M}_{1}\right)$, prescription drug use $\left(\mathrm{M}_{2}\right)$, and outpatient surgery $\left(\mathrm{M}_{3}\right)$. The outcome variable is the functional limitations score $(\mathrm{Y})$. The covariates controlled for are age, gender, race/ethnicity, education, coupled household, household assets, work status, number of health conditions, and recent injury. Paths between levels of $X$ and $M_{1}-M_{3}$ are indicated by the coefficient $a$, paths between mediating variables and $\mathrm{Y}$ are indicated by the coefficient $b$, and paths between levels of $\mathrm{X}$ and $\mathrm{Y}$ are given coefficients containing the letter $c$. For the dichotomous mediators, Rx and Surgery, two paths between M and Y were estimated. The first path serves as a reference to the second path. Effects for the reference paths are not calculated because their coefficients are estimated as zero in the model.

Modeling Longitudinal Outcomes Using Regression Analysis. A generalized linear mixed model was used to assess the effects of insurance status, doctor visits, prescription drug usage, and outpatient surgery on functional outcomes. This model type was also used to assess the effect of insurance status on each of the mediating variables doctor visits, prescription drug usage, and outpatient surgery. Mixed models are suitable for modeling longitudinal data where repeated measurements on subjects are typically correlated. Fixed effects models assume that all measurements are independent; however, this assumption is violated in data sets with repeated measures on individuals. Mixed effects regression models account for autocorrelation in intra-individual measurements by modeling one overall group mean and estimating subject-specific deviations from this mean (Arrandale, Koehoorn, MacNab, \& Kennedy, 2006).

For each step of the mediation analysis, regression models were run using SAS PROC GLIMMIX. Dichotomous outcome variables were modeled using a binary 


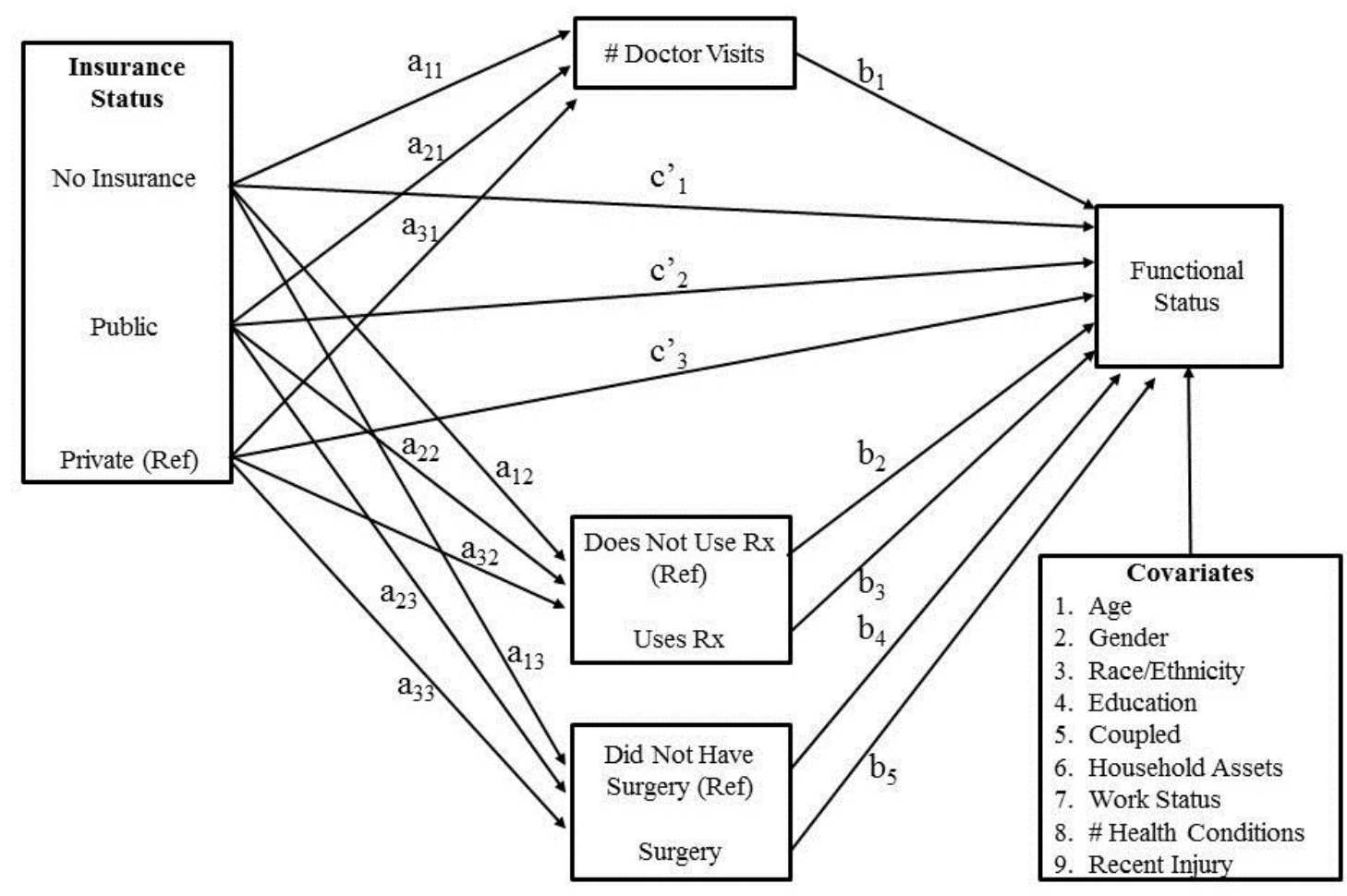

Figure 3-2. Path Diagram for the Mediation Model 
response distribution and continuous outcome variables were modeled using a normal response distribution. Data from six survey waves spaced two years apart were used in this analysis. Data from wave 1 was collected pre-injury, wave 2 was collected periinjury, and waves 3-6 were collected post-injury. Time was treated as a random effect and a first-order autoregressive heterogeneous covariance structure was selected for the model. The autoregressive covariance structure assumes that correlations between repeated measurements diminish over time, which would be expected as subjects are recovering from injury during the course of the study. Models comparing autoregressive homogenous to autoregressive heterogeneous covariance structures were also run and indicated that the heterogeneous structure resulted in a better fit to the data.

All covariates from the conceptual framework were included in the final model, even if they were not statistically significant in all models. Covariates included age, gender, race/ethnicity, education, coupled household status, household assets, work status, number of health conditions, and insurance status. Interaction effects between the independent variable and each mediating variable were also evaluated. All estimated effects used in the mediation analysis are time-varying, lower-level fixed effects. Respondents' insurance status is permitted to change over time in the model, which is common in this sample since most study respondents are approaching retirement age and eventually enroll in Medicare. Model details are described below.

\section{- Model 1: Functional Limitations Score Model without Mediators (Continuous} Outcome)

- Level 1 (Functional Limitations Score $)=$ Insurance Status + Age + Couple Status + Total Household Assets + Work Status + Number of Health Conditions + Recent Injury + Time

○ Level 2 (Functional Limitations Score $)=$ Gender + Race/Ethnicity + Education

- Model 2: Total Doctor Visits Model (Mediator 1, Continuous Outcome)

- Level 1 (Total Doctor Visits $)=$ Insurance Status + Age + Couple Status + Total Household Assets + Work Status + Number of Health Conditions + Recent Injury + Time

○ Level 2 (Total Doctor Visits) $=$ Gender + Race/Ethnicity + Education

- Model 3: Prescription Drug Model (Mediator 2, Binary Outcome)

- Level $1(\mathrm{Rx})=$ Insurance Status + Age + Couple Status + Total Household Assets + Work Status + Number of Health Conditions + Recent Injury + Time ○ Level $2(\mathrm{Rx})=$ Gender + Race/Ethnicity + Education 
- Model 4: Outpatient Surgery Model (Mediator 3, Binary Outcome)

○ Level 1 (Surgery) $=$ Insurance Status + Age + Couple Status + Total Household Assets + Work Status + Number of Health Conditions + Recent Injury + Time

○ Level 2 (Surgery) $=$ Gender + Race/Ethnicity + Education

- Model 5: Functional Limitations Score Model with Mediators (Continuous Outcome)

- Level 1 (Functional Limitations Score $)=$ Insurance Status + Total Doctor Visits $+\mathrm{Rx}+$ Surgery + Age + Couple Status + Total Household Assets + Work Status + Number of Health Conditions + Recent Injury + Time

- Level 2 (Functional Limitations Score $)=$ Gender + Race/Ethnicity + Education

Mediation Analysis with a Multicategorical Independent Variable. In this study, the independent variable of interest, insurance status, is multicategorical. Because of this, the standard mediation effects $a, c$, and $c$ ' cannot be estimated because there is not a single unit difference in $\mathrm{X}$ when it is multicategorical as compared to when $\mathrm{X}$ is continuous or dichotomous. The solution is to employ dummy coding so that coefficients quantifying differences between two discrete categories of $\mathrm{X}$ can be obtained. As a result, the total, direct, and indirect effects are considered to be relative effects (Hayes \& Preacher 2013). Relative effects differ in that they quantify the effect of one group in reference to another group. For this analysis, the reference level of $\mathrm{X}$ is private insurance and the relative effects of no insurance, $c_{1}$, and public insurance, $c_{2}$, will be calculated.

By including the multicategorical variable insurance status in the class statement of the GLIMMIX procedure, dummy coding is performed by default during the modeling process. However, dummy variables for insurance status were created in order to perform post hoc standard error calculations, which are described below.

Mediation Analysis with Multiple Mediating Variables. The conceptual framework for this study proposed that medical care use acts as a mediator between insurance status and functional outcomes. Three variables that describe various aspects of medical care use are doctor visits, prescription drug usage, and outpatient surgery. Each of these variables was included as a mediator in the analysis. A three-mediator model requires that the effect of $\mathrm{X}$ on $\mathrm{M}$ be modeled in separate regressions for each model. This results in separate mediated effects that are specific to each mediator. The total mediated effect could then be computed by finding the sum of the individual mediated effects (e.g., $a_{1} b_{1}+a_{2} b_{2}+a_{3} b_{3}=c-c^{\prime}$ ). Because this study investigated the effects of a multicategorical independent variable, separate individual mediated effects and total mediated effects was calculated for each level of X. 
Conducting Mediation Analysis. Mediation was assessed using a four-step process described by Baron and Kenny (1986). Step 1 of the mediation analysis examined whether insurance status, $\mathrm{X}$, was significantly associated with functional status, Y. This step reveals the total effect of $\mathrm{X}$ on $\mathrm{Y}$, which may or may not be significant. Because there are different levels of $\mathrm{X}$ in this analysis, the relative effects of no insurance and public insurance compared to private insurance were evaluated.

- Model 1: Y $=\mathrm{i}_{1}+c_{1} \mathrm{INSTYPE}_{1}+c_{2} \mathrm{INSTYPE}_{2}+b_{\text {age }} \mathrm{AGE}+b_{g e n} \mathrm{GEN}+$ $b_{\text {race }} \mathrm{RACE}+b_{e d u} \mathrm{EDU}+b_{\text {couple }} \mathrm{COUPLE}+b_{\text {asset }} \mathrm{ASSET}+b_{\text {work }} \mathrm{WORK}+$ $b_{\text {injury }} \mathrm{INJURY}+b_{\text {cond }} \mathrm{COND}+b_{\text {time }} \mathrm{TIME}+\mathrm{e}_{\mathrm{y}}$

Step 2 determined whether insurance status, $X$, is significantly associated with the mediating variables, $\mathrm{M}_{1}-\mathrm{M}_{3}$. The continuous variable DRVISIT is $\mathrm{M}_{1}$, and the dichotomous variables RX and SURG are $\mathrm{M}_{2}$ and $\mathrm{M}_{3}$, respectively.

- $\quad$ Model 2: $\mathrm{M}_{1}=\mathrm{i}_{2}+a_{11} \mathrm{INSTYPE}_{1}+a_{21} \mathrm{INSTYPE}_{2}+b_{\text {age }} \mathrm{AGE}+b_{\text {gen }} \mathrm{GEN}+$ $b_{\text {race }} \mathrm{RACE}+b_{\text {edu }} \mathrm{EDU}+b_{\text {couple }} \mathrm{COUPLE}+b_{\text {asset }} \mathrm{ASSET}+b_{\text {work }} \mathrm{WORK}+$ $b_{\text {injury }} \mathrm{INJURY}+b_{\text {cond }} \mathrm{COND}+b_{\text {time }}$ TIME $+\mathrm{e}_{\mathrm{m} 1}$

- Model 3: $\mathrm{M}_{2}=\mathrm{i}_{3}+a_{12} \mathrm{INSTYPE}_{1}+a_{22} \mathrm{INSTYPE}_{2}+b_{\text {age }} \mathrm{AGE}+b_{\text {gen }} \mathrm{GEN}+$ $b_{\text {race }} \mathrm{RACE}+b_{\text {edu }} \mathrm{EDU}+b_{\text {couple }} \mathrm{COUPLE}+b_{\text {asset }} \mathrm{ASSET}+b_{\text {work }} \mathrm{WORK}+$ $b_{\text {injury }} \mathrm{INJURY}+b_{\text {cond }} \mathrm{COND}+b_{\text {time }} \mathrm{TIME}+\mathrm{e}_{\mathrm{m} 2}$

- $\quad$ oodel 4: $\mathrm{M}_{3}=\mathrm{i}_{4}+a_{13} \mathrm{INSTYPE}_{1}+a_{23} \mathrm{INSTYPE}_{2}+b_{\text {age }} \mathrm{AGE}+b_{\text {gen }} \mathrm{GEN}+$ $b_{\text {race }} \mathrm{RACE}+b_{e d u} \mathrm{EDU}+b_{\text {couple }} \mathrm{COUPLE}+b_{\text {asset }} \mathrm{ASSET}+b_{\text {work }} \mathrm{WORK}+$ $b_{\text {injury }} \mathrm{INJURY}+b_{\text {cond }} \mathrm{COND}+b_{\text {time }} \mathrm{TIME}+\mathrm{e}_{\mathrm{m} 3}$

Step 3 determined whether the mediators are significantly associated with functional status while controlling for $\mathrm{X}$.

- Model 5: $\mathrm{Y}=\mathrm{i}_{5}+c{ }_{1} \mathrm{INSTYPE}_{1}+c^{\prime}{ }_{2} \mathrm{INSTYPE}_{2}+b_{1} \mathrm{DRVISIT}+b_{3} \mathrm{RX}_{2}+$ $b_{5} \mathrm{SURG}_{2}+b_{\text {age }} \mathrm{AGE}+b_{\text {gen }} \mathrm{GEN}+b_{\text {race }} \mathrm{RACE}+b_{\text {edu }} \mathrm{EDU}+b_{\text {couple }} \mathrm{COUPLE}+$ $b_{\text {asset }} \mathrm{ASSET}+b_{\text {work }} \mathrm{WORK}+b_{\text {injury }} \mathrm{INJURY}+b_{\text {cond }} \mathrm{COND}+b_{\text {time }} \mathrm{TIME}+\mathrm{e}_{\mathrm{y}}$

Step 4 determined whether the effect of $\mathrm{X}$ on $\mathrm{Y}$ is altered by including the mediating variable into the model. Results from Model 5 were used to assess the effect of insurance status on functional status. Evidence for mediation exists when the association between insurance status and functional status is reduced in the presence of the medical care use.

Comparable Estimate and Standard Error Calculations. In mediation analyses that model both binary and continuous variables, coefficients are produced in different scales. In order to create coefficients that are comparable, parameter estimates and standard errors must be rescaled using the procedure described by Mackinnon and Dwyer (1993). This required modifying Equations 3-1 through 3-5 (Table 3-3; Figure 3-3). Note that these equations have been simplified and do not include covariates from 
Table 3-3. Equations for Rescaled Outcome Variables

\begin{tabular}{ccc}
\hline Model & Original Equation & Modified Equation \\
\hline Model 1 & $\mathrm{Y}=\mathrm{c}_{1} \mathrm{X}_{1}+\mathrm{c}_{2} \mathrm{X}_{2}$ & $\mathrm{Y}^{\prime}=\mathrm{c}_{1} \mathrm{X}_{1}+\mathrm{c}_{2} \mathrm{X}_{2}$ \\
Model 2 & $\mathrm{M}_{1}=\mathrm{a}_{11} \mathrm{X}_{1}+\mathrm{a}_{21} \mathrm{X}_{2}$ & $\mathrm{M}_{1}^{\prime}=\mathrm{a}_{11} \mathrm{X}_{1}+\mathrm{a}_{21} \mathrm{X}_{2}$ \\
Model 3 & $\mathrm{M}_{2}=\mathrm{a}_{12} \mathrm{X}_{1}+\mathrm{a}_{22} \mathrm{X}_{2}$ & $\mathrm{M}_{2}{ }_{2}=\mathrm{a}_{12} \mathrm{X}_{1}+\mathrm{a}_{22} \mathrm{X}_{2}$ \\
Model 4 & $\mathrm{M}_{3}=\mathrm{a}_{13} \mathrm{X}_{1}+\mathrm{a}_{23} \mathrm{X}_{2}$ & $\mathrm{M}_{3}^{\prime}=\mathrm{a}_{13} \mathrm{X}_{1}+\mathrm{a}_{23} \mathrm{X}_{2}$ \\
Model 5 & $\mathrm{Y}=\mathrm{b}_{1} \mathrm{M}_{1}+\mathrm{b}_{3} \mathrm{M}_{2}+\mathrm{b}_{5} \mathrm{M}_{3}+\mathrm{c}_{1}{ }^{\prime} \mathrm{X}_{1}+\mathrm{c}_{2}{ }^{\prime} \mathrm{X}_{2} \quad \mathrm{Y}^{\prime \prime}=\mathrm{b}_{1} \mathrm{M}_{1}+\mathrm{b}_{3} \mathrm{M}_{2}+\mathrm{b}_{5} \mathrm{M}_{3}+\mathrm{c}_{1}{ }^{\prime} \mathrm{X}_{1}+\mathrm{c}_{2}{ }^{\prime} \mathrm{X}_{2}$ \\
\hline
\end{tabular}




$$
\operatorname{std}\left(Y^{\prime}\right)=\sqrt{c^{\prime} 1^{2} \operatorname{var}\left(X_{1}\right)+c^{\prime}{ }^{2}{ }^{2} \operatorname{var}\left(X_{2}\right)+2 c^{\prime}{ }_{1} c^{\prime}{ }_{2} \operatorname{cov}\left(X_{1}, X_{2}\right)+\frac{\pi^{2}}{3}}
$$

(Equation 3-1)

$\operatorname{std}\left(Y^{\prime \prime}\right)$

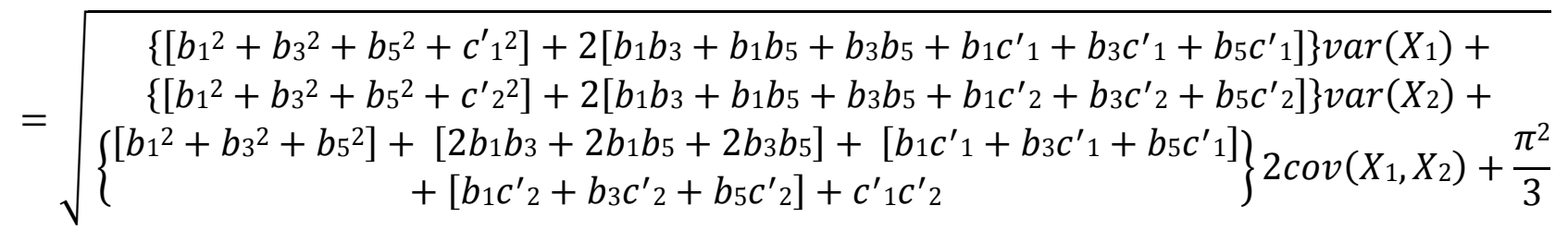

\section{(Equation 3-2)}

$$
\operatorname{std}\left(M_{1}{ }^{\prime}\right)=\sqrt{a_{11^{2}} \operatorname{var}\left(X_{1}\right)+a_{21}{ }^{2} \operatorname{var}\left(X_{2}\right)+2 a_{11} a_{21} \operatorname{cov}\left(X_{1}, X_{2}\right)+\frac{\pi^{2}}{3}}
$$

$$
\operatorname{std}\left(M_{2}^{\prime}\right)=\sqrt{a_{12^{2}} \operatorname{var}\left(X_{1}\right)+a_{22^{2}} \operatorname{var}\left(X_{2}\right)+2 a_{12} a_{22} \operatorname{cov}\left(X_{1}, X_{2}\right)+\frac{\pi^{2}}{3}}
$$

\section{(Equation 3-3)}

\section{(Equation 3-4)}

$$
\operatorname{std}\left(M_{3^{\prime}}\right)=\sqrt{a_{13^{2}} \operatorname{var}\left(X_{1}\right)+a_{23^{2}} \operatorname{var}\left(X_{2}\right)+2 a_{13} a_{23} \operatorname{cov}\left(X_{1}, X_{2}\right)+\frac{\pi^{z}}{3}}
$$

(Equation 3-5)

\section{Figure 3-3. Equations for Calculating Standard Deviations for $Y^{\prime}, Y^{\prime}, M_{1}{ }^{\prime}, M_{2}$ ', and $M_{3}$ '}


the original model. Additionally, $\mathrm{X}_{1}$ and $\mathrm{X}_{2}$ correspond to the dummy coded insurance status variables. $\mathrm{X}_{1}$ indicates the dummy coded variable for No Insurance and $\mathrm{X}_{2}$ indicates Public Insurance.

The prime indicates that outcome variables in the modified equations are on a different scale than the original model. To compute the comparable coefficients, the standard deviation of $\mathrm{X}_{1}, \mathrm{X}_{2}, \mathrm{M}_{1}, \mathrm{M}_{1}$ ', $\mathrm{M}_{2}, \mathrm{M}_{2}$ ', $\mathrm{M}_{3}, \mathrm{M}_{3}$ ', $\mathrm{Y}$ ', and $\mathrm{Y}$ ' ' must be obtained. Standard deviations for Y', Y',, $\mathrm{M}_{1}$ ', $\mathrm{M}_{2}$ ', and $\mathrm{M}_{3}$ ' were calculated using Equations 3-1 through 3-5 (Figure 3-3), which were derived from variance formulas described in MacKinnon and Dwyer (1993). Standard deviations of $\mathrm{X}_{1}, \mathrm{X}_{2}, \mathrm{M}_{1}, \mathrm{M}_{2}$, and $\mathrm{M}_{3}$ were obtained from conventional descriptive analyses.

Once the standard deviations were calculated, the comparable coefficients and comparable standard errors for each path were calculated using the formulas in Figures 3-4 and 3-5.

Determining Relative Total, Direct, and Indirect Effects. Because the mediation model contains both dichotomous and continuous outcome variables, parameter estimates were rescaled in order to produce comparable coefficients and standard errors across models. The rescaled estimates were then used to calculate each of the effects listed below. Each relative individual indirect effect was calculated by finding the product of coefficients between the corresponding level of X and M. Relative total indirect effects were computed by finding the sum of each individual indirect effect for a given level of X. Effects were not calculated for paths containing reference levels of categorical variables because all coefficients were set to zero.

- Relative Individual Indirect Effects

Indirect Effect of No Insurance and Doctor Visits $=a_{11} b_{1}$

$\circ \quad$ Indirect Effect of No Insurance and $R x=a_{12} b_{3}$

$\circ$ Indirect Effect of No Insurance and Surgery $=a_{13} b_{5}$

Indirect Effect of Public Insurance and Doctor Visits $=a_{21} b_{1}$

$O$ Indirect Effect of Public Insurance and $R x=a_{22} b_{3}$

$\circ$ Indirect Effect of Public Insurance and Surgery $=a_{23} b_{5}$

- Relative Total Indirect Effects

O Total Indirect Effect of No Insurance $=a_{11} b_{1}+a_{12} b_{3}+a_{13} b_{5}$

○ Total Indirect Effect of Public Insurance $=a_{21} b_{1}+a_{22} b_{3}+a_{23} b_{5}$

- Relative Direct Effects

- Direct Effect of No Insurance $=\mathrm{c}^{\prime} 1$

$\circ$ Direct Effect of Public Insurance $=c^{\prime}{ }_{2}$ 


$$
\begin{array}{ll}
\text { comparable } a_{11}=a_{11} \times \frac{s t d\left(X_{1}\right)}{s t d\left(M_{1}{ }^{\prime}\right)} & \text { comparable } b_{3}=b_{3} \times \frac{s t d\left(M_{2}\right)}{s t d\left(Y^{\prime \prime}\right)} \\
\text { comparable } a_{12}=a_{12} \times \frac{s t d\left(X_{1}\right)}{s t d\left(M_{2}{ }^{\prime}\right)} & \text { comparable } b_{5}=b_{5} \times \frac{s t d\left(M_{3}\right)}{s t d\left(Y^{\prime \prime}\right)} \\
\text { comparable } a_{13}=a_{13} \times \frac{s t d\left(X_{1}\right)}{s t d\left(M_{3}\right)} & \text { comparable } c_{1}=c_{1} \times \frac{s t d\left(X_{1}\right)}{s t d\left(Y^{\prime}\right)} \\
\text { comparable } a_{21}=a_{21} \times \frac{s t d\left(X_{2}\right)}{s t d\left(M_{1}{ }^{\prime}\right)} & \text { comparable } c_{2}=c_{2} \times \frac{s t d\left(X_{2}\right)}{s t d\left(Y^{\prime}\right)} \\
\text { comparable } a_{22}=a_{22} \times \frac{s t d\left(X_{2}\right)}{s t d\left(M_{2}{ }^{\prime}\right)} & \text { comparable } c^{\prime}{ }_{1}=c^{\prime}{ }_{1} \times \frac{s t d\left(X_{1}\right)}{s t d\left(Y^{\prime \prime}\right)} \\
\text { comparable } a_{23}=a_{23} \times \frac{s t d\left(X_{2}\right)}{s t d\left(M_{3}{ }^{\prime}\right)} & \text { comparable } c^{\prime}{ }_{2}=c^{\prime}{ }_{2} \times \frac{s t d\left(X_{2}\right)}{s t d\left(Y^{\prime \prime}\right)} \\
\text { comparable } b_{1}=b_{1} \times \frac{s t d\left(M_{1}\right)}{s t d\left(Y^{\prime \prime}\right)} &
\end{array}
$$

Figure 3-4. Formulas for Comparable Coefficients 


$$
\begin{aligned}
& \text { comparable se }\left(a_{11}\right)=\operatorname{se}\left(a_{11}\right) \times \frac{s t d\left(X_{1}\right)}{s t d\left(M_{1}^{\prime}\right)} \quad \text { comparable se }\left(b_{3}\right)=\operatorname{se}\left(b_{3}\right) \times \frac{s t d\left(M_{2}\right)}{s t d\left(Y^{\prime \prime}\right)} \\
& \text { comparable se }\left(a_{12}\right)=\operatorname{se}\left(a_{12}\right) \times \frac{s t d\left(X_{1}\right)}{s t d\left(M_{2}^{\prime}\right)} \quad \text { comparable se }\left(b_{5}\right)=\operatorname{se}\left(b_{5}\right) \times \frac{s t d\left(M_{3}\right)}{s t d\left(Y^{\prime \prime}\right)} \\
& \text { comparable se }\left(a_{13}\right)=\operatorname{se}\left(a_{13}\right) \times \frac{s t d\left(X_{1}\right)}{s t d\left(M_{3}^{\prime}\right)} \quad \text { comparable se }\left(c_{1}\right)=\operatorname{se}\left(c_{1}\right) \times \frac{s t d\left(X_{1}\right)}{s t d\left(Y^{\prime}\right)} \\
& \text { comparable se }\left(a_{21}\right)=\operatorname{se}\left(a_{21}\right) \times \frac{s t d\left(X_{2}\right)}{s t d\left(M_{1}^{\prime}\right)} \quad \text { comparable se }\left(c_{2}\right)=\operatorname{se}\left(c_{2}\right) \times \frac{s t d\left(X_{2}\right)}{s t d\left(Y^{\prime}\right)} \\
& \text { comparable se }\left(a_{22}\right)=\operatorname{se}\left(a_{22}\right) \times \frac{s t d\left(X_{2}\right)}{s t d\left(M_{2}{ }^{\prime}\right)} \quad \text { comparable se }\left(c^{\prime}{ }_{1}\right)=\operatorname{se}\left(c^{\prime}{ }_{1}\right) \times \frac{\operatorname{std}\left(X_{1}\right)}{\operatorname{std}\left(Y^{\prime \prime}\right)} \\
& \text { comparable se }\left(a_{23}\right)=\operatorname{se}\left(a_{23}\right) \times \frac{s t d\left(X_{2}\right)}{s t d\left(M_{3}{ }^{\prime}\right)} \quad \text { comparable se }\left(c^{\prime}{ }_{2}\right)=\operatorname{se}\left(c^{\prime}{ }_{2}\right) \times \frac{\operatorname{std}\left(X_{2}\right)}{\operatorname{std}\left(Y^{\prime \prime}\right)} \\
& \text { comparable se }\left(b_{1}\right)=\operatorname{se}\left(b_{1}\right) \times \frac{\operatorname{std}\left(M_{1}\right)}{s t d\left(Y^{\prime \prime}\right)}
\end{aligned}
$$

Figure 3-5. Formulas for Comparable Standard Errors 
- Relative Total Effects

- Total Effect of No Insurance $=\mathrm{c}_{1}$

$\circ$ Total Effect of Public Insurance $=c_{2}$

Formulas for standard error calculations for the relative individual indirect effects, relative total indirect effects, and relative total effects are below. The formula for direct effects was described previously.

Standard errors for relative individual indirect effects of No Insurance are as follows:

$$
\begin{aligned}
& \operatorname{se}\left(a_{11} b_{1}\right)=\sqrt{a_{11^{2}} s e\left(b_{1}\right)^{2}+b_{1}^{2} s e\left(a_{11}\right)^{2}} \\
& \operatorname{se}\left(a_{12} b_{3}\right)=\sqrt{a_{12^{2}} s e\left(b_{3}\right)^{2}+b_{3}^{2} s e\left(a_{12}\right)^{2}} \\
& \operatorname{se}\left(a_{13} b_{5}\right)=\sqrt{a_{13^{2}} \operatorname{se}\left(b_{5}\right)^{2}+b_{5}^{2} \operatorname{se}\left(a_{13}\right)^{2}}
\end{aligned}
$$

Standard errors for relative individual indirect effects of Public Insurance are as follows:

$$
\begin{aligned}
& \operatorname{se}\left(a_{21} b_{1}\right)=\sqrt{a_{21}^{2} s e\left(b_{1}\right)^{2}+b_{1}^{2} s e\left(a_{21}\right)^{2}} \\
& \operatorname{se}\left(a_{22} b_{3}\right)=\sqrt{a_{22^{2}} s e\left(b_{3}\right)^{2}+b_{3}^{2} s e\left(a_{22}\right)^{2}} \\
& \operatorname{se}\left(a_{23} b_{5}\right)=\sqrt{a_{23^{2}} \operatorname{se}\left(b_{5}\right)^{2}+b_{5}^{2} \operatorname{se}\left(a_{23}\right)^{2}}
\end{aligned}
$$

Standard error for the relative total indirect effects of No Insurance is as follows:

$$
\begin{aligned}
\operatorname{se}\left(c^{\prime}{ }_{1}+a_{11} b_{1}\right. & \left.+a_{12} b_{3}+a_{13} b_{5}\right) \\
& =\sqrt{\begin{array}{c}
\operatorname{se}\left(a_{11} b_{1}\right)^{2}+\operatorname{se}\left(a_{12} b_{3}\right)^{2}+\operatorname{se}\left(a_{13} b_{5}\right)^{2}+2 a_{11} a_{12} \operatorname{cov}\left(b_{1}, b_{3}\right)+ \\
2 a_{11} a_{13} \operatorname{cov}\left(b_{1}, b_{5}\right)+2 a_{12} a_{13} \operatorname{cov}\left(b_{3}, b_{5}\right)
\end{array}}
\end{aligned}
$$

Standard error for the relative total indirect effects of Public Insurance is as follows:

$$
\begin{aligned}
s e\left(c^{\prime}{ }_{2}+a_{21} b_{1}\right. & \left.+a_{22} b_{3}+a_{23} b_{5}\right) \\
& =\sqrt{\begin{array}{c}
s e\left(a_{21} b_{1}\right)^{2}+\operatorname{se}\left(a_{22} b_{3}\right)^{2}+\operatorname{se}\left(a_{23} b_{5}\right)^{2}+2 a_{21} a_{22} \operatorname{cov}\left(b_{1}, b_{3}\right)+ \\
2 a_{21} a_{23} \operatorname{cov}\left(b_{1}, b_{5}\right)+2 a_{22} a_{23} \operatorname{cov}\left(b_{3}, b_{5}\right)
\end{array}}
\end{aligned}
$$

After determining the comparable effect estimates and standard errors for all effects, $95 \%$ confidence intervals were computed for each effect using these formulas:

$$
\begin{aligned}
& \text { Lower } 95 \% \text { confidence limit }(L C L)=\text { effect }-1.96 \text { se }(\text { effect }) \\
& \text { Upper } 95 \% \text { confidence limit }(U C L)=\text { effect }+1.96 \operatorname{se}(\text { effect })
\end{aligned}
$$




\section{CHAPTER 4. RESULTS}

\section{Introduction}

This chapter presents the results of all study analyses. First, it will provide a description of the study sample and all study variables. Next, it will present findings from the trajectory analysis and results comparing trajectory predictor variables. These results will be followed by a description of the findings from the mediation analysis. Finally, all results will be applied to study hypotheses.

\section{Study Sample Description}

Baseline characteristics of the injured HRS study sample are shown in Table 4-1, along with characteristics of non-injured HRS respondents. The injured sample consisted of 591 survey respondents. Overall the study sample was predominantly white $(77.5 \%)$, attained at least a high school diploma (75\%), and live with their spouses $(76.5 \%)$. Nearly $80 \%$ of the sample was under 65 years of age at baseline, with just over a fifth of the sample being 55 or younger. Because the majority of the sample is under 65 at baseline, only $29.8 \%$ are publicly insured. Approximately $60 \%$ of study participants have private insurance as their primary insurance and only $8.6 \%$ have no form of health insurance. Most study participants were still working at baseline (61.9\%). The median total household assets for the study sample was $\$ 101,100$.

\section{Comparison of Injured Cohort to Total HRS Sample}

Compared to non-injured HRS respondents, the injured sample was significantly younger, had a greater proportion of males, had a greater number of high school graduates, and was more likely to be living with a spouse. The injured sample was also more likely to be currently working at baseline. A much larger portion of non-injured HRS respondents were publicly insured, likely because a greater number were over 65 and eligible for Medicare. Additionally, the non-injured respondents had a greater number of health conditions compared to the injured sample.

\section{Missing Cases}

In general, the HRS has a high follow-up rate with survey participants and attrition is very low. In this sample, over $98 \%$ of cases are complete for the first four waves of data (Table 4-2). For waves 5 and 6, the number of missing cases increases to $8.8 \%$ and $13.5 \%$, respectively. Because the majority of cases were complete over the first four waves of the study and the sample is an elderly population, attrition is likely due to mortality rather than refusal to re-interview. 
Table 4-1. Descriptive Characteristics at Baseline

\begin{tabular}{|c|c|c|c|}
\hline Variable & $\begin{array}{c}\text { Non-Injured HRS } \\
\text { Respondents } \\
(\mathbf{2 0 , 7 9 3 )} \\
\end{array}$ & $\begin{array}{c}\text { Injured HRS } \\
\text { Study Sample } \\
(n=591)\end{array}$ & $\begin{array}{c}p \\
\text { Value }\end{array}$ \\
\hline Age Group & & & $<0.001$ \\
\hline 55 and Under & $3,285(15.8)$ & $122(20.6)$ & \\
\hline $56-60$ & $3,680(17.7)$ & $180(30.5)$ & \\
\hline $61-65$ & $3,469(16.7)$ & $176(29.8)$ & \\
\hline $66-70$ & $2,938(14.1)$ & $88(14.9)$ & \\
\hline 71 and Older & $7,421(35.7)$ & $25(4.2)$ & \\
\hline Gender & & & 0.026 \\
\hline Male & $8,688(41.8)$ & $274(46.4)$ & \\
\hline Female & $12,105(58.2)$ & $317(53.6)$ & \\
\hline Race/Ethnicity & & & 0.433 \\
\hline White & $15,862(76.3)$ & $458(77.5)$ & \\
\hline Black & $2,889(13.9)$ & $80(13.5)$ & \\
\hline Hispanic & $1,600(7.7)$ & $47(8.0)$ & \\
\hline Other & $413(2.0)$ & $6(1.0)$ & \\
\hline Education & & & 0.006 \\
\hline Less than HS & $6,311(30.4)$ & $148(25.0)$ & \\
\hline HS Diploma & $6,907(33.2)$ & $229(38.7)$ & \\
\hline Some College & $3,844(18.5)$ & $120(20.3)$ & \\
\hline Bachelor's Degree or Higher & $3,672(17.7)$ & $94(15.9)$ & \\
\hline Lives in a Coupled Household & $14,063(67.6)$ & $452(76.5)$ & $<0.001$ \\
\hline Currently Working & $7,092(34.2)$ & $366(61.9)$ & $<0.001$ \\
\hline Household Assets, median & $\$ 117,600$ & $\$ 101,100$ & 0.261 \\
\hline Insurance Type & & & $<0.001$ \\
\hline Private & $8,066(38.8)$ & $360(60.9)$ & \\
\hline Public & $11,455(55.1)$ & $176(29.8)$ & \\
\hline No Insurance & $1,132(5.4)$ & $51(8.6)$ & \\
\hline Number of Health Conditions & & & 0.046 \\
\hline None & $4,746(22.8)$ & $151(25.5)$ & \\
\hline One & $6,223(29.9)$ & $193(32.7)$ & \\
\hline Two & $5,060(24.3)$ & $136(23.0)$ & \\
\hline Three or More & $4,763(22.9)$ & $111(18.8)$ & \\
\hline
\end{tabular}

The number and percentage of respondents within levels a categorical variable are reported. 
Table 4-2. Number and Percentage of Cases Missing across Survey Waves

\begin{tabular}{lcccccc}
\hline & \multicolumn{6}{c}{ Survey Wave } \\
\cline { 2 - 7 } \multicolumn{1}{c}{ Variable } & $\mathbf{1 9 9 8}$ & $\mathbf{2 0 0 0}$ & $\mathbf{2 0 0 2}$ & $\mathbf{2 0 0 4}$ & $\mathbf{2 0 0 6}$ & $\mathbf{2 0 0 8}$ \\
\hline $\begin{array}{l}\text { Number of } \\
\text { Missing Cases }\end{array}$ & $0(0.0)$ & $0(0.0)$ & $10(1.7)$ & $3(0.5)$ & $52(8.8)$ & $80(13.5)$ \\
\hline
\end{tabular}




\section{Main Study Variables}

\section{Functional Limitations Scale Creation}

In order to create the functional limitations scale, the dependent variable in this study, a factor analysis was conducted to evaluate scale items for each year of data. For each wave, only a single eigenvalue was greater than one, indicating that there was a single factor linking all scale items together (Table 4-3).

After confirming that there was only one factor based on eigenvalues, a reliability analysis was conducted. The Cronbach's Alphas for each item being included in the scale and also deleted from the scale were computed. Alpha values ranged from 0.79 to 0.81 for the scale, indicating very good reliability. None of the alpha values for deleted items were substantially higher than the all-item scale and therefore each item was included in the final scale (Table 4-4).

\section{Functional Status over Time}

Between 1998 and 2000, there is an increase in mean functional limitations for each functional status measure. Changes in the mobility, large muscle, and gross motor skills indices indicate that respondents experience an increase in functional limitations during the peri-injury period and do not return to baseline functioning during the postinjury period. However, the fine motor skills index does show an increase in functional limitations during the peri-injury period that degrades over time. Similarly, the percentage of respondents indicating that pain causes difficulty performing activities increases after injury and then returns to near baseline levels by the first post-injury measurement. After the start of the post-injury period, all measures of functional limitation gradually increase with each successive survey wave, with the exception of the 2008 pain measure (Table 4-5).

\section{Independent Variables over Time}

Over the course of the study, the proportion of participants with private insurance declines and the number of participants with public insurance increases. At baseline, $61.3 \%$ of respondents are privately insured, $30.0 \%$ of respondents are publicly insured, and $8.7 \%$ have no insurance. It appears that for each wave, the proportion of respondents with private insurance decreases by approximately $10 \%$ and the proportion of respondents with public insurance increases by approximately $10 \%$. Additionally, the number of people with no insurance gradually decreases throughout the study. By the final survey wave, nearly $80 \%$ of participants have public insurance, while only $17.7 \%$ are privately insured and 3\% are uninsured (Table 4-6). 
Table 4-3. Functional Limitations Scale Factor Analysis

\begin{tabular}{lcccccc}
\hline & \multicolumn{6}{c}{ Eigenvalues } \\
\cline { 2 - 7 } \multicolumn{1}{c}{ Outcome Variable } & $\mathbf{1 9 9 8}$ & $\mathbf{2 0 0 0}$ & $\mathbf{2 0 0 2}$ & $\mathbf{2 0 0 4}$ & $\mathbf{2 0 0 6}$ & $\mathbf{2 0 0 8}$ \\
\hline Mobility Index & 3.005 & 2.941 & 3.103 & 3.028 & 3.108 & 3.103 \\
Large Muscle Index & 0.776 & 0.855 & 0.739 & 0.76 & 0.754 & 0.761 \\
Fine Motor Skills Index & 0.68 & 0.606 & 0.527 & 0.565 & 0.543 & 0.558 \\
Gross Motor Skills Index & 0.411 & 0.471 & 0.489 & 0.521 & 0.469 & 0.458 \\
$\begin{array}{l}\text { Difficulty Performing Activities } \\
\text { Due to Pain }\end{array}$ & 0.127 & 0.128 & 0.143 & 0.125 & 0.126 & 0.12 \\
\hline
\end{tabular}

Table 4-4. Reliability Analysis for Functional Limitations Scale Items

\begin{tabular}{lccccccc}
\hline & \multicolumn{6}{c}{ Cronbach's Alpha } \\
\cline { 2 - 7 } \multicolumn{1}{c}{ Outcome Variable } & $\mathbf{1 9 9 8}$ & $\mathbf{2 0 0 0}$ & $\mathbf{2 0 0 2}$ & $\mathbf{2 0 0 4}$ & $\mathbf{2 0 0 6}$ & $\mathbf{2 0 0 8}$ \\
\hline Functional Limitations Scale & 0.79 & 0.79 & 0.80 & 0.81 & 0.81 & 0.81 \\
& \multicolumn{6}{c}{ Cronbach's Alpha if Item Deleted } \\
\cline { 2 - 7 } Mobility Index & 0.68 & 0.69 & 0.70 & 0.70 & 0.72 & 0.71 \\
Large Muscle Index & 0.75 & 0.76 & 0.77 & 0.77 & 0.78 & 0.78 \\
Fine Motor Skills Index & 0.81 & 0.79 & 0.80 & 0.81 & 0.81 & 0.81 \\
Gross Motor Skills Index & 0.70 & 0.69 & 0.70 & 0.70 & 0.71 & 0.71 \\
Difficulty Performing Activities & & & & & & \\
Due to Pain & 0.79 & 0.80 & 0.80 & 0.82 & 0.81 & 0.82 \\
\hline
\end{tabular}


Table 4-5. Functional Outcomes across Survey Waves

\begin{tabular}{|c|c|c|c|c|c|c|}
\hline \multirow[b]{3}{*}{ Outcome Variable } & \multicolumn{6}{|c|}{ Survey Wave } \\
\hline & \multirow{2}{*}{$\begin{array}{c}\text { Pre-Injury } \\
1998\end{array}$} & \multirow{2}{*}{$\begin{array}{c}\text { Peri- } \\
\text { Injury } \\
\mathbf{2 0 0 0}\end{array}$} & \multicolumn{4}{|c|}{ Post-Injury } \\
\hline & & & 2002 & 2004 & 2006 & 2008 \\
\hline Mobility Index & $0.85(1.31)$ & $1.18(1.48)$ & $1.19(1.43)$ & $1.26(1.51)$ & $1.39(1.53)$ & $1.36(1.58)$ \\
\hline Large Muscle Index & $1.23(1.37)$ & $1.56(1.38)$ & $1.56(1.37)$ & $1.57(1.31)$ & $1.66(1.32)$ & $1.64(1.34)$ \\
\hline $\begin{array}{l}\text { Fine Motor Skills Index } \\
\text { Gross Motor Skills }\end{array}$ & $0.14(0.43)$ & $0.24(0.54)$ & $0.16(0.45)$ & $0.22(0.57)$ & $0.23(0.58)$ & $0.23(0.56)$ \\
\hline Index & $0.37(0.92)$ & $0.53(1.07)$ & $0.51(1.05)$ & $0.60(1.19)$ & $0.62(1.14)$ & $0.66(1.25)$ \\
\hline Difficulty Performing & & & & & & \\
\hline $\begin{array}{l}\text { Activities Due to Pain, } \\
\mathrm{n}(\%)\end{array}$ & $137(23.2)$ & $245(41.5)$ & $150(25.4)$ & $156(26.4)$ & $158(26.7)$ & $145(24.5)$ \\
\hline Functional Limitations & 16.49 & 24.50 & 20.88 & 22.06 & 23.74 & 23.50 \\
\hline Scale & $(21.31)$ & $(23.24)$ & $(22.46)$ & $(23.11)$ & $(23.82)$ & $(24.10)$ \\
\hline
\end{tabular}

Mean values are reported for all scale variables with standard deviations contained in the parentheses. The number and percentage of respondents are reported for categorical variables. 
Table 4-6. Insurance Status across Survey Waves

Survey Wave

\begin{tabular}{|c|c|c|c|c|c|c|}
\hline \multirow[b]{3}{*}{ Insurance Status } & \multicolumn{6}{|c|}{ Survey Wave } \\
\hline & \multirow{2}{*}{$\begin{array}{c}\text { Pre-Injury } \\
1998\end{array}$} & \multirow{2}{*}{$\begin{array}{c}\begin{array}{c}\text { Peri- } \\
\text { Injury }\end{array} \\
2000\end{array}$} & \multicolumn{4}{|c|}{ Post-Injury } \\
\hline & & & 2002 & 2004 & 2006 & 2008 \\
\hline Private Insurance & $360(61.3)$ & $306(51.8)$ & $234(40.4)$ & $176(29.9)$ & $123(23.3)$ & $89(17.7)$ \\
\hline Public Insurance & $176(30.0)$ & $235(39.8)$ & $297(51.3)$ & $376(63.8)$ & $383(72.5)$ & $400(79.4)$ \\
\hline No Insurance & $51(8.7)$ & $50(8.5)$ & $48(8.3)$ & $37(6.3)$ & $22(4.2)$ & $15(3.0)$ \\
\hline
\end{tabular}




\section{Mediating Variables over Time}

Between 1998 and 2008, most indicators of medical care use increase over time, with the greatest increase occurring between the pre-injury and peri-injury periods (Table 4-7). The mean number of doctor visits increases from 9 to 12 between these periods and the standard deviation for doctor visits also increases between these two periods (18 to 27). The standard deviation for this measure is highest throughout the course of the study during the peri-injury period (27). This indicates that the greatest variation in medical care use occurs during this period. The mean number of doctor visits does decrease slightly during the post-injury period, but does not return to pre-injury levels.

Additionally, the proportion of the sample using prescription drugs increases throughout the entire course of the study. Between the pre-injury and peri-injury periods, there is nearly a $10 \%$ increase in the number of participants using prescription medication $(67.7 \%$ to $77.0 \%)$. This measure does not return to pre-injury levels during the recovery period, but rather gradually increases.

Lastly, the percentage of participants undergoing outpatient surgery increases slightly between the pre-injury and peri-injury period (21.0\% to $25.7 \%$ ), and then returns close to pre-injury levels during the first wave of the post-injury period $(20.5 \%)$. However, overall, the percentage of participants undergoing surgery is relatively constant throughout the 10-year study.

\section{Correlations among Study Variables}

Table 4-8 presents a correlation matrix for all study variables. Nearly all correlations between demographic, socioeconomic, insurance status, and functional status were significant. Functional status was significantly correlated with doctor visits and prescription drug use, but less so with surgery. Insurance status was correlated with several demographic variables including age, education, work status, and coupled households, but not with gender and race/ethnicity. Additionally, insurance status was only significantly correlated with household assets during the first three waves of the study. The medical care use variables doctor visits and prescription drug use were both strongly correlated with work status, but with few of the other demographic and socioeconomic variables. However, prescription drug use was significantly correlated to age and gender, unlike doctor visits and surgery. Additionally, doctor visits and prescription drug use were correlated to one another. Surgery had the fewest number of significant correlations among the medical care use variables, with prescription drug use and number of medical conditions being the most consistently substantial relationships across survey waves. Lastly, the number of medical conditions a respondent had was significantly correlated with almost all variables with the exception of gender and race. 
Table 4-7. Mediating Variables across Survey Waves

\begin{tabular}{|c|c|c|c|c|c|c|}
\hline \multirow{3}{*}{ Mediators } & \multicolumn{6}{|c|}{ Survey Wave } \\
\hline & \multirow{2}{*}{$\begin{array}{c}\begin{array}{c}\text { Pre- } \\
\text { Injury }\end{array} \\
1998 \\
\end{array}$} & \multirow{2}{*}{$\begin{array}{c}\begin{array}{c}\text { Peri- } \\
\text { Injury }\end{array} \\
2000 \\
\end{array}$} & \multicolumn{4}{|c|}{ Post-Injury } \\
\hline & & & 2002 & 2004 & 2006 & 2008 \\
\hline Doctor Visits & $9(18)$ & $12(27)$ & $11(19)$ & $11(16)$ & $12(19)$ & $10(12)$ \\
\hline \multicolumn{7}{|l|}{$\mathrm{Rx}$} \\
\hline Use $R x$ & $400(67.7)$ & $455(77.0)$ & $452(77.8)$ & $475(80.5)$ & $453(84.2)$ & $433(84.7)$ \\
\hline Do Not Use Rx & $191(32.3)$ & $136(23.0)$ & $129(22.2)$ & $115(19.5)$ & 85 (15.8) & $78(15.3)$ \\
\hline \multicolumn{7}{|l|}{ Surgery } \\
\hline Had Surgery & $124(21.0)$ & $152(25.7)$ & $119(20.5)$ & $138(23.4)$ & $117(21.7)$ & $114(22.3)$ \\
\hline No Surgery & $467(79.0)$ & $439(74.3)$ & $462(79.5)$ & $452(76.6)$ & $421(78.3)$ & $397(77.7)$ \\
\hline
\end{tabular}


Table 4-8. Correlation among All Study Variables

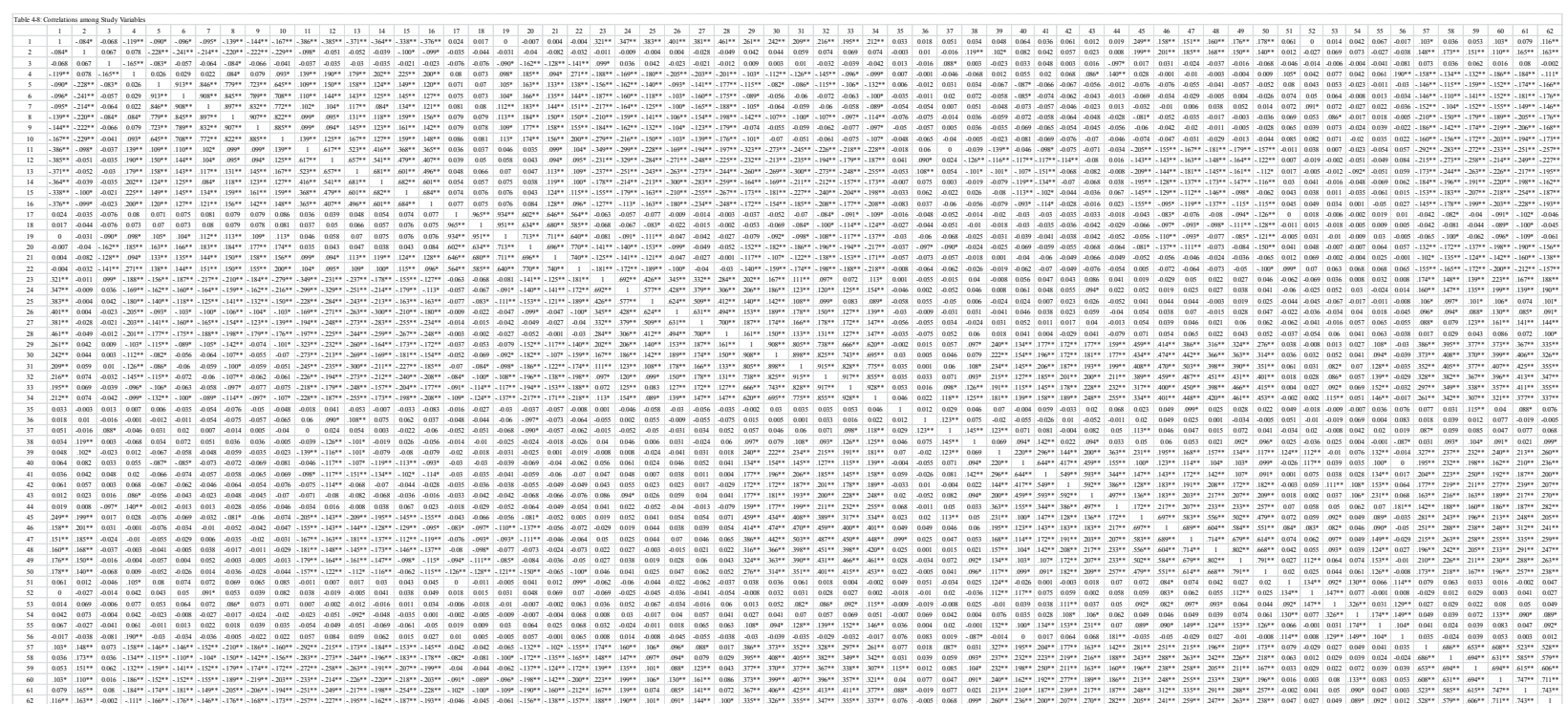

Note: Must be magnified to be readable. 


\section{Part I. Classification of Functional Status Trajectory after Injury and Identification of Predictors of Long-Term Functional Outcomes}

Identifying Recovery Subgroups Using a Group-Based Trajectory Model. The modeling process using PROC TRAJ is iterative and finding the best fit model requires a priori decisions to be made using substantive knowledge about the data and research area. The first step of the modeling process required identifying a theoretical maximum number of subgroups. After determining a priori that five subgroups would be the maximum, the optimum number of subgroups was determined by successively fitting models with 1, 2, 3, 4, and 5 trajectories. All group orders were set as quadratic and BIC values for each model were used to calculate the change in BIC between simpler and more complex models (Table 4-9). The differences in BIC values were then used to calculate Bayes factors (Table 4-10). The Bayes factors were interpreted using Jeffrey's Scale of Evidence (Table 4-11).

Based on Jeffrey's Scale of Evidence for Bayes Factors, a trajectory model with 5 subgroups was selected. The Bayes factor comparing Model 5 and Model 4 was $5.44 \mathrm{x}$ $10^{10}$, which indicates there is strong evidence in favor of Model 5.

Once the optimum number of subgroups was determined for the data, models comparing various trajectory shapes were run. Models were run beginning with the most basic polynomial equation (i.e., linear parameters for all subgroups) and then repeated using more complex polynomial equations until the best-fitting model was identified. The orders for each trajectory were determined based on BIC values as well as substantive knowledge of the data. After identifying the appropriate order for each subgroup, a timevarying covariate for injury was incorporated into the model. Additionally, parameter start values were adjusted to reduce the likelihood of a local solution to the equations.

The final model consisted of two linear trajectories, a quadratic trajectory, and two cubic trajectories. All parameters in the final model were significant. The first figure below compares the mean functional limitations score with the predicted value for each subgroup across time (Figure 4-1). The predicted values closely align with the subgroup mean values. Predicted values deviate most from the mean values during the peri-injury period, indicating that the greatest variation in functional status scores occurs at this time point. The second figure contains predicted values for functional status scores and $95 \%$ confidence intervals for each trajectory (Figure 4-2). Trajectories 1, 2, and 5 are stratified and are generally parallel to one another. Trajectories 2 and 3 have overlapping functional limitations scores at baseline, but gradually diverge over time. Trajectories 3 and 4 cross over one another during the course of the study and overlap during waves 3 and 4 . Trajectory means along with predicted values and $95 \%$ confidence intervals are presented in tabular for in Table 4-12. Finally, Table 4-13 shows the number of respondents who were assigned to each trajectory. The greatest percentage of respondents belongs to Trajectory $2(46.3 \%)$ and the percentage of respondents belonging to the other 4 trajectories range between $10.5 \%$ and $18.9 \%$. 
Table 4-9. BIC Values for Trajectory Models

\begin{tabular}{ccc}
\hline Model & No. of Trajectories & BIC \\
\hline Model 1 & $\mathrm{k}=1$ & -13124.33 \\
Model 2 & $\mathrm{k}=2$ & -12485.91 \\
Model 3 & $\mathrm{k}=3$ & -12286.56 \\
Model 4 & $\mathrm{k}=4$ & -12202.24 \\
Model 5 & $\mathrm{k}=5$ & -12177.52 \\
\hline
\end{tabular}

Table 4-10. Bayes Factors for Trajectory Models

\begin{tabular}{ccc}
\hline Comparison & BIC $_{\mathbf{i}}-\mathbf{B I C}_{\mathbf{j}}$ & $\begin{array}{c}\text { Bayes } \\
\text { Factor }\end{array}$ \\
\hline Model 2 - Model 1 & 638.42 & $1.83 \times 10^{227}$ \\
Model 3 - Model 2 & 199.35 & $3.77 \times 10^{86}$ \\
Model 4 - Model 3 & 84.32 & $4.17 \times 10^{36}$ \\
Model 5 - Model 4 & 24.72 & $5.44 \times 10^{10}$ \\
\hline
\end{tabular}

Table 4-11. Jeffrey's Scale of Evidence for Bayes Factors

\begin{tabular}{ll}
\hline \multicolumn{1}{c}{ Bayes Factor } & \multicolumn{1}{c}{ Interpretation } \\
\hline$B i j<1 / 10$ & Strong evidence for model $j$ \\
$1 / 10<B i j<1 / 3$ & Moderate evidence for model $j$ \\
$1 / 3<B i j<3$ & Weak evidence for model $j$ \\
$3<B i j<10$ & Moderate evidence for model $i$ \\
$B i j>10$ & Strong evidence for model $i$ \\
\hline
\end{tabular}




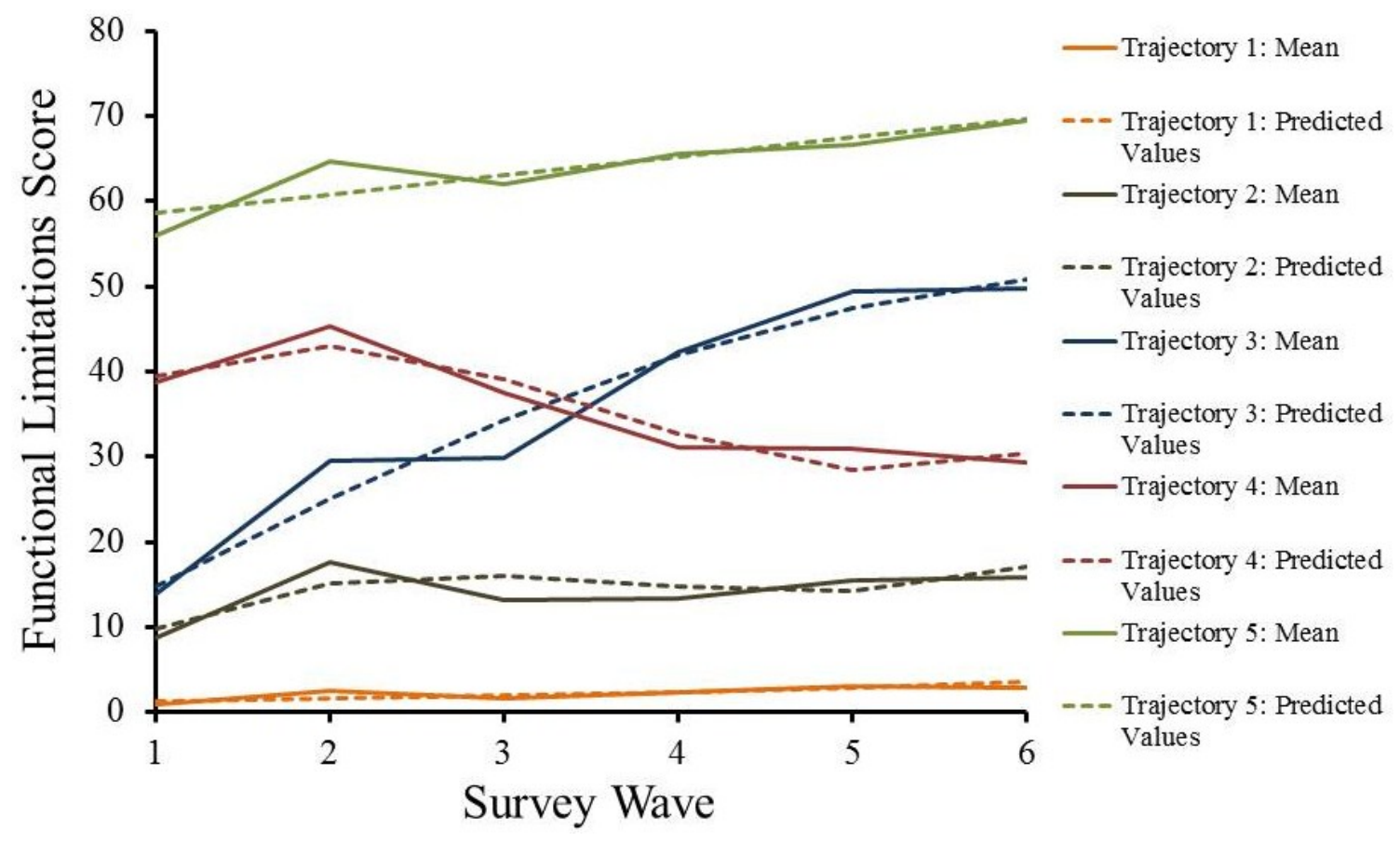

Figure 4-1. Mean and Predicted Functional Limitations Scores across Survey Waves 


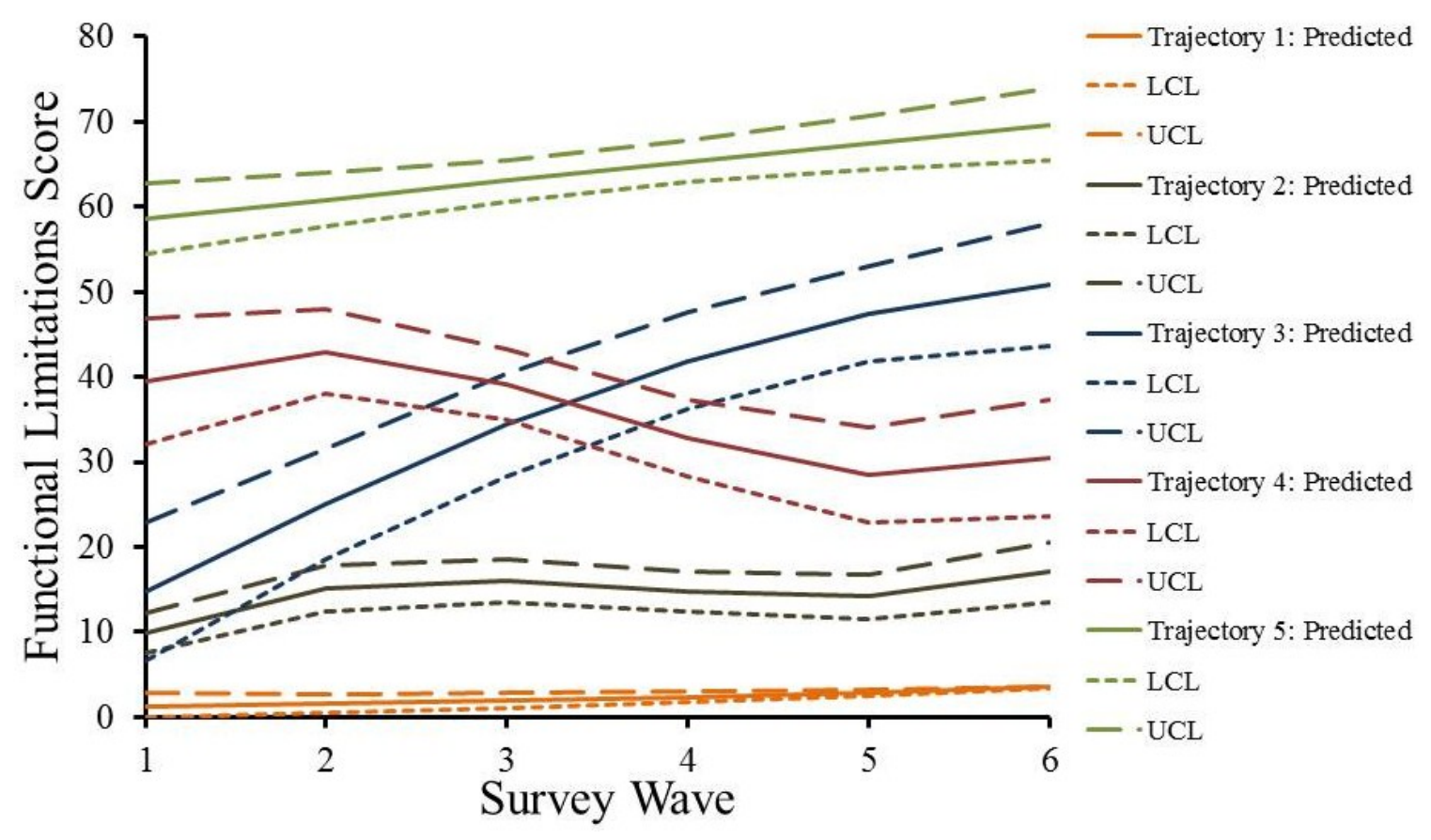

Figure 4-2. Predicted Functional Limitations Scores and 95\% Confidence Intervals across Survey Waves 
Table 4-12. Mean and Predicted Functional Limitations Scores

\begin{tabular}{|c|c|c|c|c|c|c|c|c|c|c|c|c|}
\hline \multirow[b]{2}{*}{ Trajectory } & \multicolumn{4}{|c|}{1998} & \multicolumn{4}{|c|}{2000} & \multicolumn{4}{|c|}{2002} \\
\hline & Mean & Pred & LCL & UCL & Mean & Pred & LCL & UCL & Mean & Pred & LCL & UCL \\
\hline 1 & 0.8 & 1.3 & 0.0 & 2.8 & 2.5 & 1.6 & 0.5 & 2.7 & 1.5 & 2.0 & 1.2 & 2.8 \\
\hline 2 & 8.8 & 9.9 & 7.6 & 12.2 & 17.7 & 15.1 & 12.5 & 17.8 & 13.2 & 16.0 & 13.5 & 18.5 \\
\hline 3 & 13.9 & 14.8 & 6.7 & 22.9 & 29.5 & 25.0 & 18.5 & 31.5 & 29.9 & 34.4 & 28.4 & 40.3 \\
\hline 4 & 38.8 & 39.5 & 32.1 & 46.9 & 45.4 & 42.9 & 38.0 & 47.9 & 37.4 & 39.2 & 35.0 & 43.3 \\
\hline 5 & 56.1 & 58.6 & 54.5 & 62.7 & 64.7 & 60.9 & 57.7 & 64.0 & 62.0 & 63.1 & 60.6 & 65.6 \\
\hline
\end{tabular}

Table 4-12. Continued

\begin{tabular}{|c|c|c|c|c|c|c|c|c|c|c|c|c|}
\hline \multirow[b]{2}{*}{ Trajectory } & \multicolumn{4}{|c|}{2004} & \multicolumn{4}{|c|}{2006} & \multicolumn{4}{|c|}{2008} \\
\hline & Mean & Pred & LCL & UCL & Mean & Pred & LCL & UCL & Mean & Pred & LCL & UCL \\
\hline 1 & 2.3 & 2.4 & 1.8 & 3.0 & 3.1 & 2.9 & 2.6 & 3.3 & 3.0 & 3.5 & 3.5 & 3.5 \\
\hline 2 & 13.4 & 14.8 & 12.4 & 17.2 & 15.4 & 14.2 & 11.6 & 16.8 & 15.8 & 17.0 & 13.5 & 20.6 \\
\hline 3 & 42.3 & 41.9 & 36.2 & 47.6 & 49.4 & 47.4 & 41.8 & 53.0 & 49.9 & 50.8 & 43.6 & 58.0 \\
\hline 4 & 31.1 & 32.8 & 28.2 & 37.4 & 30.9 & 28.5 & 22.9 & 34.1 & 29.4 & 30.5 & 23.6 & 37.4 \\
\hline 5 & 65.6 & 65.3 & 62.9 & 67.7 & 66.7 & 67.5 & 64.4 & 70.6 & 69.5 & 69.7 & 65.4 & 74.0 \\
\hline
\end{tabular}


Table 4-13. Number and Percentage of Respondents Belonging to Each Trajectory Subgroup

\begin{tabular}{ccc}
\hline Trajectory & $\mathbf{n}$ & $\mathbf{\%}$ \\
\hline 1 & 112 & 18.9 \\
2 & 274 & 46.3 \\
3 & 62 & 10.5 \\
4 & 79 & 13.4 \\
5 & 64 & 10.8 \\
\hline
\end{tabular}


Characteristics of Trajectory Subgroups. The group-based trajectory model identified 5 distinct trajectories for the injured study sample. The graph below depicts the average functional limitations score for each group across time and the trajectory label assigned to each (Figure 4-3). Following is a description of each trajectory, based on its pattern of change in functional limitations scores over time.

- Trajectory 1: Static, High Functioning - This group is characterized as having a consistently low score on the functional limitations scale.

- Trajectory 2: Dynamic, Big Hit - This group is characterized as having an initial increase in functional limitations after injury followed by a gradual recovery over time (i.e. decreasing functional limitations score). However, these individuals do not return to their pre-injury level of functioning and continue to have some functional limitations during the post-injury period.

- Trajectory 3: Dynamic, Slow Burn - This group is characterized as having an initial increase in functional limitations after injury followed by a further decline in functional status over time (i.e. increasing functional limitations score).

- Trajectory 4: Dynamic, Long-Term Improvement- This group is characterized as having an increase in functional limitations during the peri-injury period followed by gradual improvement. These individuals' have fewer functional limitations during the post-injury period than the pre-injury period.

- Trajectory 5: Static, Low Functioning - This group is characterized as having a consistently high score on the functional limitations scale.

Four of the trajectories that were identified by the model were similar to the hypothesized subgroups described in Chapter 3. Trajectories 1, 2, 3, and 5 demonstrate a pattern of change that fits the descriptions of trajectories proposed by Iwashyna (2012). However, a fifth trajectory (i.e. Trajectory 4) differed from the hypothesized subgroups. Rather than fluctuating in functional status over time, resulting in a flat, intermediate trajectory, Trajectory 4 appears to experience an initial functional deficit during the periinjury period but then goes on to consistently improve over time. This subgroup has a similar pattern of change to Trajectory 2 (Big Hit), but Trajectory 4 has a significantly higher functional limitations score at baseline. Additionally, members of Trajectory 4 improve during the post-injury period and eventually have a lower functional limitations score than they had at baseline.

Both of the static trajectories (1 and 5) had fairly flat functional limitations slopes over the course of the study. Both increased slightly during the peri-injury period, but were relatively stationary across time. These two subgroups also differed significantly from one another throughout the study, with Trajectory 5 having a much higher average functional limitations score than Trajectory 1.

Two of the dynamic trajectories ( 3 and 4 ) had relatively large changes in functional status over the course of the study. Trajectory 3 , in particular, tends to gradually decline over time and continues to acquire more functional limitations in the post-injury period. Trajectory 3 has mean functional limitations scores of 14.0 at the preinjury time point, 29.5 at the peri-injury time point, and 49.9 during the final time point 


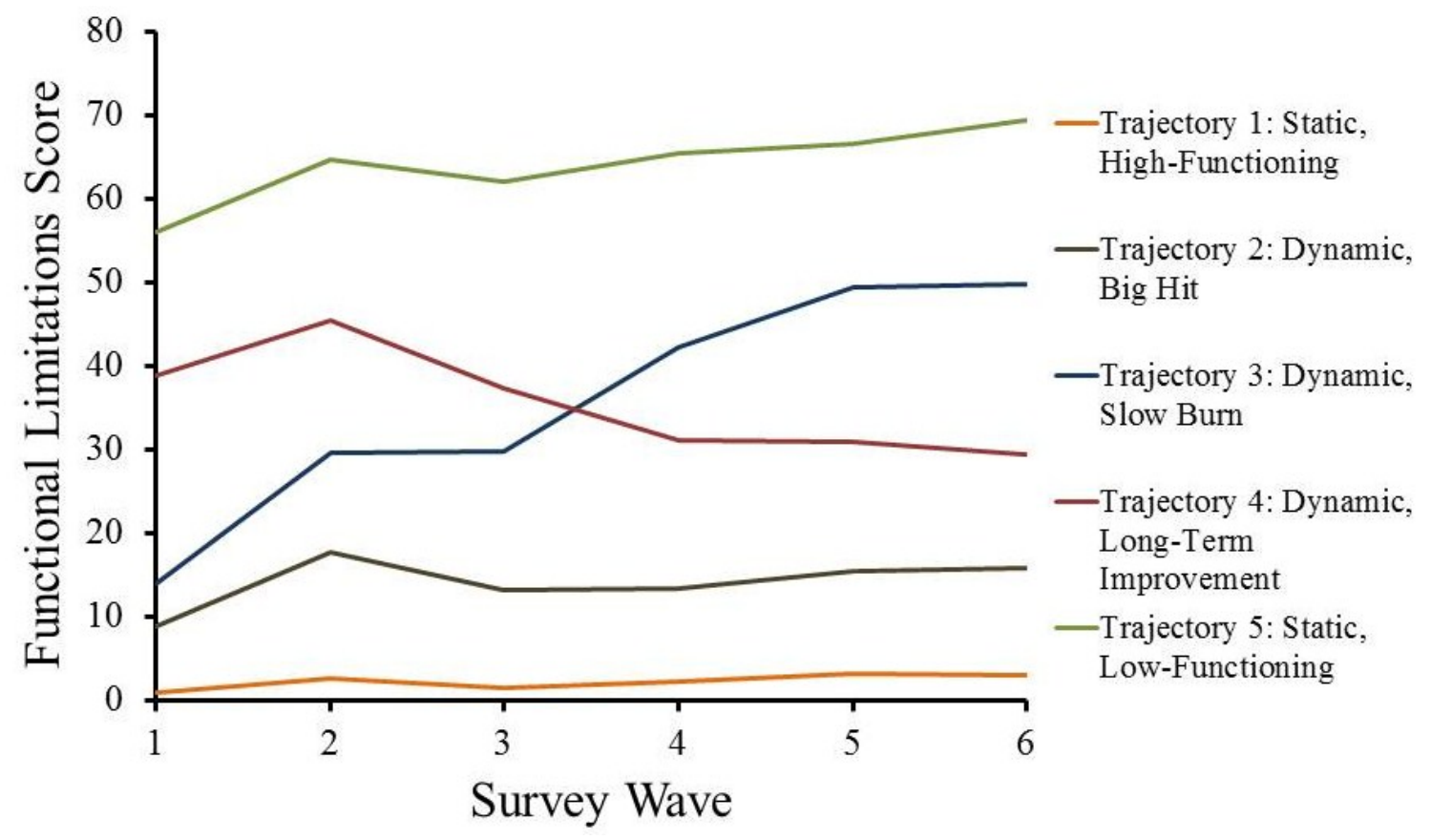

Figure 4-3. Trajectory Mean Functional Limitations Scores across Survey Waves 
of the post-injury period. In contrast, Trajectory 4 improves over time and has mean functional limitations scores of 38.8 at baseline, 45.4 during the peri-injury period, and 29.4 at the final time point.

Trajectory 2 is most similar to the hypothesized "Big Hit" trajectory, although there is only a small increase in mean functional limitations between the pre-injury and peri-injury time points (8.8 and 17.7). However, this subgroup's functional limitation score does decrease during the post-injury period, indicating that some functional improvement has possibly taken place.

Baseline descriptive statistics for each subgroup are shown below in Table 4-14. The trajectory subgroups differed significantly on all baseline characteristics, with the exception of age and race/ethnicity $(\mathrm{p}=0.083$ and 0.284$)$. Trajectory 1 had a greatest percentage of members under $65(89.1 \%)$ whereas approximately $80 \%$ of members were under the age of 65 in all other trajectories. The largest majority of female members belonged to Trajectories 4 and $5(62.2 \%$ and 69.2\%). Trajectory 1 had the largest percentage of males $(60.9 \%)$ and Trajectories 2 and 3 had a relatively even number of males and females. A significantly greater number of people in Trajectories 1 and 2 had Bachelor's degrees (22.7\% and 18.5\%) compared to Trajectories 3, 4, and $5(6.6 \%, 9.5 \%$, and $9.2 \%$ ). $44.6 \%$ of Trajectory 5 did not live in a coupled household, whereas as the other trajectories ranged between 19.1\% and 27.9\%. Most members belonging to Trajectories 1,2 , and 3 were currently working at baseline $(90.9 \%, 62.1 \%$, and $62.3 \%)$, while only $44.6 \%$ of Trajectory 4 and $32.3 \%$ of Trajectory 5 were working at the start of the study. Individuals in Trajectory 1 tended to fall in the higher household assets quintiles and those in Trajectory 5 fell into quintiles that had relatively fewer assets. In fact, $67.7 \%$ of people in Trajectory 5 were in either the low or lowest asset quintiles. With the exception of Trajectory 1, the majority of members in each trajectory had at least 1 health condition. Over half of the people in Trajectory 5 had 3 or more health conditions $(52.3 \%)$. Lastly, most trajectories were made up predominantly of people with private insurance. Trajectory 5 had the greatest number of members with public insurance $(61.9 \%)$ and Trajectory 3 had the greatest number of uninsured members $(19.7 \%)$.

Insurance Status and Medical Care Use over Time in Trajectory Subgroups. For all trajectory subgroups, the proportion of individuals with private insurance gradually decreased over time while the proportion with public insurance increased over time. However, the percentage of uninsured participants fluctuated over time for several specific trajectories. For Trajectory 1, the number of people without insurance does not change between the pre-injury and peri-injury periods; however, it increases during the post-injury period. Trajectory 2 also experiences an increase in the number of uninsured members during the first wave of the post-injury period. Although it has the greatest proportion of uninsured members at baseline, the percentage of uninsured members decreases between 1998 and 2004 for Trajectory 3. This is followed by a slight increase during the last two waves of the study. Trajectory 4 has an increase in the percentage of uninsured members during the peri-injury period, but this is followed by a gradual decrease for the remainder of the study. Lastly, Trajectory 5 has a constant decrease in the proportion of uninsured members throughout the entire study. 
Table 4-14. Baseline Characteristics for Trajectory Subgroup

\begin{tabular}{|c|c|c|c|c|c|c|}
\hline Variable & $\begin{array}{c}\text { Trajectory } \\
1 \\
\mathbf{n}=112 \\
\end{array}$ & $\begin{array}{c}\text { Trajectory } \\
2 \\
\mathrm{n}=274 \\
\end{array}$ & $\begin{array}{c}\text { Trajectory } \\
3 \\
n=62 \\
\end{array}$ & $\begin{array}{c}\text { Trajectory } \\
4 \\
n=79 \\
\end{array}$ & $\begin{array}{c}\text { Trajectory } \\
5 \\
n=64 \\
\end{array}$ & p Value \\
\hline Age Category & & & & & & 0.083 \\
\hline 55 and Under & $31.8 \%$ & $19.2 \%$ & $21.3 \%$ & $12.2 \%$ & $16.9 \%$ & \\
\hline $56-60$ & $28.2 \%$ & $31.0 \%$ & $32.8 \%$ & $35.1 \%$ & $24.6 \%$ & \\
\hline $61-65$ & $29.1 \%$ & $28.1 \%$ & $24.6 \%$ & $33.8 \%$ & $38.5 \%$ & \\
\hline $66-70$ & $10.0 \%$ & $16.4 \%$ & $19.7 \%$ & $14.9 \%$ & $12.3 \%$ & \\
\hline 71 and Older & $0.9 \%$ & $5.3 \%$ & $1.6 \%$ & $4.1 \%$ & $7.7 \%$ & \\
\hline Gender & & & & & & 0.001 \\
\hline Male & $60.9 \%$ & $46.6 \%$ & $45.9 \%$ & $37.8 \%$ & $30.8 \%$ & \\
\hline Female & $39.1 \%$ & $53.4 \%$ & $54.1 \%$ & $62.2 \%$ & $69.2 \%$ & \\
\hline Race/Ethnicity & & & & & & 0.284 \\
\hline White & $86.4 \%$ & $76.9 \%$ & $80.3 \%$ & $74.3 \%$ & $66.2 \%$ & \\
\hline Black & $5.5 \%$ & $13.9 \%$ & $14.8 \%$ & $14.9 \%$ & $23.1 \%$ & \\
\hline Hispanic & $7.3 \%$ & $8.2 \%$ & $4.9 \%$ & $9.5 \%$ & $9.2 \%$ & \\
\hline Other & $0.9 \%$ & $1.1 \%$ & $0.0 \%$ & $1.4 \%$ & $1.5 \%$ & \\
\hline Education & & & & & & 0.001 \\
\hline Bachelor's Degree or & & & & & & \\
\hline Higher & $22.7 \%$ & $18.5 \%$ & $6.6 \%$ & $9.5 \%$ & $9.2 \%$ & \\
\hline Some College & $24.5 \%$ & $19.6 \%$ & $26.2 \%$ & $13.5 \%$ & $18.5 \%$ & \\
\hline HS Diploma & $39.1 \%$ & $39.1 \%$ & $34.4 \%$ & $47.3 \%$ & $30.8 \%$ & \\
\hline Less than HS & $13.6 \%$ & $22.8 \%$ & $32.8 \%$ & $29.7 \%$ & $41.5 \%$ & \\
\hline Coupled Household & & & & & & 0.001 \\
\hline Not Coupled & $19.1 \%$ & $20.3 \%$ & $27.9 \%$ & $20.3 \%$ & $44.6 \%$ & \\
\hline Coupled & $80.9 \%$ & $79.7 \%$ & $72.1 \%$ & $79.7 \%$ & $55.4 \%$ & \\
\hline
\end{tabular}


Table 4-14. Continued

\begin{tabular}{|c|c|c|c|c|c|c|}
\hline Variable & $\begin{array}{c}\text { Trajectory } \\
1 \\
n=112 \\
\end{array}$ & $\begin{array}{c}\text { Trajectory } \\
\mathbf{2} \\
\mathbf{n}=\mathbf{2 7 4} \\
\end{array}$ & $\begin{array}{c}\text { Trajectory } \\
3 \\
n=62 \\
\end{array}$ & $\begin{array}{c}\text { Trajectory } \\
3 \\
n=79 \\
\end{array}$ & $\begin{array}{c}\text { Trajectory } \\
4 \\
n=64 \\
\end{array}$ & p Value \\
\hline Work Status & & & & & & $<0.001$ \\
\hline Not Working & $9.1 \%$ & $37.9 \%$ & $37.7 \%$ & $55.4 \%$ & $67.7 \%$ & \\
\hline Currently Working & $90.9 \%$ & $62.1 \%$ & $62.3 \%$ & $44.6 \%$ & $32.3 \%$ & \\
\hline Household Assets & & & & & & $<0.001$ \\
\hline Highest Quintile & $25.5 \%$ & $23.8 \%$ & $11.5 \%$ & $12.2 \%$ & $10.8 \%$ & \\
\hline High Quintile & $22.7 \%$ & $19.9 \%$ & $18.0 \%$ & $23.0 \%$ & $13.8 \%$ & \\
\hline Medium Quintile & $20.9 \%$ & $20.6 \%$ & $29.5 \%$ & $20.3 \%$ & $7.7 \%$ & \\
\hline Low Quintile & $19.1 \%$ & $18.9 \%$ & $21.3 \%$ & $20.3 \%$ & $24.6 \%$ & \\
\hline Lowest Quintile & $11.8 \%$ & $16.7 \%$ & $19.7 \%$ & $24.3 \%$ & $43.1 \%$ & \\
\hline Number of Health Conditions & & & & & & $<0.001$ \\
\hline No Health Conditions & $63.6 \%$ & $23.8 \%$ & $9.8 \%$ & $6.8 \%$ & $4.6 \%$ & \\
\hline 1 Condition & $24.5 \%$ & $37.0 \%$ & $37.7 \%$ & $35.1 \%$ & $20.0 \%$ & \\
\hline 2 Conditions & $10.0 \%$ & $25.6 \%$ & $29.5 \%$ & $27.0 \%$ & $23.1 \%$ & \\
\hline 3 or More Conditions & $1.8 \%$ & $13.5 \%$ & $23.0 \%$ & $31.1 \%$ & $52.3 \%$ & \\
\hline Insurance Status & & & & & & $<0.001$ \\
\hline Private Insurance & $75.5 \%$ & $66.3 \%$ & $54.1 \%$ & $54.1 \%$ & $30.2 \%$ & \\
\hline Public Insurance & $16.4 \%$ & $26.2 \%$ & $26.2 \%$ & $40.5 \%$ & $61.9 \%$ & \\
\hline No Insurance & $8.2 \%$ & $7.5 \%$ & $19.7 \%$ & $5.4 \%$ & $7.9 \%$ & \\
\hline
\end{tabular}


Over the course of the study, the average number of doctor visits increases for each of the trajectory subgroups. The change in the number of doctor visits between the pre-injury and peri-injury time points is greatest for Trajectory 3 (6.3 to 12.7). For Trajectory 4 , the number of doctor visits increases during the peri-injury period and this increase remains for the first wave of the post-injury period before decreasing back to pre-injury levels. Trajectory 5 consistently has the highest number of doctor visits throughout study. Additionally, its highest average occurs during the peri-injury period.

Similar to the average number of doctor visits over time, the percentage of individuals using prescription drugs increases throughout the study. Trajectory 1 has a large increase in the number of members using prescription drugs between the pre-injury and peri-injury waves $(39.1 \%$ to $50.9 \%)$. After this increase, the percentage of prescription drug users continues to grow over the course of the study, but with smaller incremental increases between waves. Trajectory 3 also has a large increase in the percentage of people using medication between the pre-injury and peri-injury waves $(68.9 \%$ to $83.6 \%)$. This is followed by an additional $10.4 \%$ increase during the first postinjury wave. The percentage of Trajectory 4 members using medication increases from $78.4 \%$ to $89.2 \%$ during the first two waves of the study and remains somewhat stable for the rest of the study. Overall, Trajectory 5 has the greatest percentage of medication use, with over $92 \%$ of members using prescription drugs at each time point of the study. However, there is an increase from $92.3 \%$ to $100 \%$ between the first two waves. After this, approximately $98 \%$ of members remain on medication for the rest of the study.

Compared to medication use, a much smaller percentage of participants report having surgery at each wave of the study. The increase in percentage of people having outpatient surgery between the pre-injury and post-injury time points is greatest for Trajectories 2 and 5 (19.9\% to $28.8 \%$ and $26.2 \%$ to $36.9 \%$ ). Both Trajectories 3 and 4 have decreases during this time frame $(21.3 \%$ to $18.0 \%$ and $20.3 \%$ to $16.2 \%)$. For Trajectories 1,2 , and 5 , the percentage of members undergoing surgery during the postinjury period is generally similar to the percentage having surgery in the pre-injury period. Trajectories 3 and 4, however, show somewhat of an increase in surgery during the post-injury period compared to the pre-injury and peri-injury periods.

Table 4-15 displays percentages and means of insurance status and medical care use variables across all waves.

\section{Determining Predictors of Recovery Subgroups}

After examining characteristics of all subgroups, logistic regression was used to determine predictors that can discriminate between trajectories that are most distinct, such as groups that show significant improvement versus significant decline in functional status over time.

Bivariate Regression Analyses. Individual regression analyses were carried out comparing Trajectories 1, 3, 4, and 5 to Trajectory 2 . Because Trajectory 2 had a 
Table 4-15. Insurance Status and Medical Care Use over Time

\begin{tabular}{|c|c|c|c|c|c|c|}
\hline \multirow[b]{2}{*}{ Variable } & \multirow{2}{*}{$\begin{array}{c}\text { Pre-Injury } \\
1998\end{array}$} & \multirow{2}{*}{$\begin{array}{c}\text { Peri- } \\
\text { Injury } \\
2000\end{array}$} & \multicolumn{4}{|c|}{ Post-Injury } \\
\hline & & & 2002 & 2004 & 2006 & 2008 \\
\hline \multicolumn{7}{|c|}{ Trajectory 1} \\
\hline \multicolumn{7}{|l|}{ Insurance Status } \\
\hline Private Insurance & $75.5 \%$ & $65.5 \%$ & $51.9 \%$ & $45.9 \%$ & $39.4 \%$ & $29.9 \%$ \\
\hline Public Insurance & $16.4 \%$ & $26.4 \%$ & $38.0 \%$ & $43.1 \%$ & $51.5 \%$ & $64.9 \%$ \\
\hline No Insurance & $8.2 \%$ & $8.2 \%$ & $10.2 \%$ & $11.0 \%$ & $9.1 \%$ & $5.2 \%$ \\
\hline Doctor Visits & $4.6(5.7)$ & $5.7(5.9)$ & $6.0(6.9)$ & $7.1(14.8)$ & $7.6(13.6)$ & $7.7(10.4)$ \\
\hline Use $\mathrm{Rx}$ & $39.1 \%$ & $50.9 \%$ & $52.8 \%$ & $57.3 \%$ & $66.3 \%$ & $65.7 \%$ \\
\hline Had Surgery & $20.9 \%$ & $21.8 \%$ & $13.0 \%$ & $18.2 \%$ & $23.8 \%$ & $23.2 \%$ \\
\hline \multicolumn{7}{|c|}{ Trajectory 2} \\
\hline \multicolumn{7}{|l|}{ Insurance Status } \\
\hline Private Insurance & $66.3 \%$ & $55.9 \%$ & $43.5 \%$ & $31.7 \%$ & $24.0 \%$ & $16.4 \%$ \\
\hline Public Insurance & $26.2 \%$ & $36.3 \%$ & $47.8 \%$ & $61.6 \%$ & $73.2 \%$ & $81.6 \%$ \\
\hline No Insurance & $7.5 \%$ & $7.8 \%$ & $8.7 \%$ & $6.8 \%$ & $2.8 \%$ & $2.0 \%$ \\
\hline Doctor Visits & $7.8(10.1)$ & $8.4(8.2)$ & $8.2(7.7)$ & $9.0(8.7)$ & $10.210 .9)$ & $8.9(8.3)$ \\
\hline Use $R x$ & $70.1 \%$ & $77.2 \%$ & $76.9 \%$ & $80.8 \%$ & $82.6 \%$ & $85.9 \%$ \\
\hline Had Surgery & $19.9 \%$ & $28.8 \%$ & $21.3 \%$ & $21.0 \%$ & $17.3 \%$ & $20.5 \%$ \\
\hline \multicolumn{7}{|c|}{ Trajectory 3} \\
\hline \multicolumn{7}{|l|}{ Insurance Status } \\
\hline Private Insurance & $54.1 \%$ & $49.2 \%$ & $41.4 \%$ & $29.5 \%$ & $20.4 \%$ & $16.0 \%$ \\
\hline Public Insurance & $26.2 \%$ & $36.1 \%$ & $50.0 \%$ & $67.2 \%$ & $74.1 \%$ & $76.0 \%$ \\
\hline No Insurance & $19.7 \%$ & $14.8 \%$ & $8.6 \%$ & $3.3 \%$ & $5.6 \%$ & $8.0 \%$ \\
\hline
\end{tabular}


Table 4-15. Continued

\begin{tabular}{|c|c|c|c|c|c|c|}
\hline \multirow[b]{2}{*}{ Variable } & \multirow{2}{*}{$\begin{array}{c}\text { Pre-Injury } \\
1998\end{array}$} & \multirow{2}{*}{$\begin{array}{c}\begin{array}{c}\text { Peri- } \\
\text { Injury }\end{array} \\
\mathbf{2 0 0 0} \\
\end{array}$} & \multicolumn{4}{|c|}{ Post-Injury } \\
\hline & & & 2002 & 2004 & 2006 & 2008 \\
\hline \multicolumn{7}{|c|}{ Trajectory 3 continued } \\
\hline Doctor Visits & $6.3(5.7)$ & $12.730 .4)$ & $11.210 .4)$ & $14.112 .7)$ & $17.930 .4)$ & $14.717 .3)$ \\
\hline Use Rx & $68.9 \%$ & $83.6 \%$ & $93.2 \%$ & $93.4 \%$ & $96.4 \%$ & $96.0 \%$ \\
\hline Had Surgery & $21.3 \%$ & $18.0 \%$ & $25.4 \%$ & $32.8 \%$ & $29.6 \%$ & $24.0 \%$ \\
\hline \multicolumn{7}{|c|}{ Trajectory 4} \\
\hline \multicolumn{7}{|l|}{ Insurance Status } \\
\hline Private Insurance & $54.1 \%$ & $44.6 \%$ & $32.4 \%$ & $17.8 \%$ & $14.9 \%$ & $12.7 \%$ \\
\hline Public Insurance & $40.5 \%$ & $47.3 \%$ & $62.2 \%$ & $78.1 \%$ & $82.1 \%$ & $85.7 \%$ \\
\hline No Insurance & $5.4 \%$ & $8.1 \%$ & $5.4 \%$ & $4.1 \%$ & $3.0 \%$ & $1.6 \%$ \\
\hline Doctor Visits & $11.414 .2)$ & $15.4(18.6)$ & $15.225 .1)$ & $9.3(7.8)$ & $11.010 .8)$ & $9.3(9.1)$ \\
\hline Use $\mathrm{Rx}$ & $78.4 \%$ & $89.2 \%$ & $87.8 \%$ & $89.0 \%$ & $95.5 \%$ & $90.5 \%$ \\
\hline Had Surgery & $20.3 \%$ & $16.2 \%$ & $23.0 \%$ & $27.4 \%$ & $23.9 \%$ & $23.8 \%$ \\
\hline \multicolumn{7}{|c|}{ Trajectory 5} \\
\hline \multicolumn{7}{|l|}{ Insurance Status } \\
\hline Private Insurance & $30.2 \%$ & $21.5 \%$ & $15.9 \%$ & $9.2 \%$ & $3.7 \%$ & $8.0 \%$ \\
\hline Public Insurance & $61.9 \%$ & $72.3 \%$ & $77.8 \%$ & $89.2 \%$ & $94.4 \%$ & $92.0 \%$ \\
\hline \multirow[t]{2}{*}{ No Insurance } & $7.9 \%$ & $6.2 \%$ & $6.3 \%$ & $1.5 \%$ & $1.9 \%$ & $0.0 \%$ \\
\hline & & 31.5 & 25.5 & 24.6 & 25.2 & 18.9 \\
\hline Doctor Visits & $25.7(44.7)$ & $(70.6)$ & $(47.2)$ & $(34.2)$ & $(40.2)$ & $(20.8)$ \\
\hline Use Rx & $92.3 \%$ & $100.0 \%$ & $98.4 \%$ & $96.9 \%$ & $98.2 \%$ & $98.0 \%$ \\
\hline Had Surgery & $26.2 \%$ & $36.9 \%$ & $22.2 \%$ & $29.2 \%$ & $28.6 \%$ & $26.0 \%$ \\
\hline
\end{tabular}


recovery pattern that was most similar to what is clinically expected and it had the largest number of members, it served as a reference group in all models. Additional models comparing Trajectory 4 with Trajectories 3 and 5 were also run.

The analysis comparing Trajectory 1 and 2 found several baseline variables that significantly predicted membership in Trajectory 1 versus Trajectory 2 . Individuals in Trajectory 1 were less likely to fall into the oldest age category, less likely to be female, more likely to not be working at baseline, and less likely to have 1 or more health conditions. The regression comparing Trajectories 3 and 2 found that respondents without a high school diploma, who belonged to the intermediate household assets quintile, who had 2 or more health conditions, and who were uninsured at baseline were more likely to belong to Trajectory 3 . Females, individuals in the lowest household assets quintile, individuals with 1 more health conditions, and individuals with public insurance were more likely to belong in Trajectory 4 that Trajectory 2 . Individuals belonging to Trajectory 5 were less likely to be over 66 years of age, more likely to be female, less likely to be not working at baseline, more likely to have 2 or more health conditions, and more likely to have public health insurance compared to individuals in Trajectory 2 . No significant predictors were found comparing Trajectory 4 with Trajectory 3 . However, these were two of the smaller subgroups and this analysis may have had less power as a result. Although not significant at the 0.05 level, several relationships were bordering on significance between these two trajectories. Individuals in Trajectory 4 may have been more likely to live in a coupled household, more likely to be working at baseline, and less likely to be uninsured than those belonging to Trajectory 3 . The regression comparing Trajectory 4 with Trajectory 5 found that individuals in Trajectory 4 were more likely to belong to older age groups and also more likely to live in a coupled household. (Table 4-16)

In general, these analyses showed that women, individuals with multiple health conditions, and individuals with public insurance were more likely to belong to trajectories with poorer functional outcomes.

Multinomial Regression Analyses. Multinomial regression was also conducted to identify significant predictors of each of the functional status trajectory subgroups. This analysis provides information about predictors of each subgroup while using the entire sample. The reference group used in the multinomial regression analysis was Trajectory 2.

Overall, the results of the multinomial regression analysis revealed findings similar to those of the bivariate regression analyses. Members of Trajectory 1 were more likely to be male, working at baseline, and less likely to have any health conditions. Individuals in Trajectory 3 were less likely to have a high school diploma, more likely to belong to the intermediate household assets quintile, more likely to have 2 or more health conditions and less likely to have health insurance. The only significant predictors comparing Trajectory 4 and 2 were household assets and health conditions. Those belonging to Trajectory 4 were more likely to be in the lowest household assets quintile 
Table 4-16. Bivariate Logistic Regression Analyses to Distinguish between Trajectory Subgroups

\begin{tabular}{|c|c|c|c|c|c|c|}
\hline & $\begin{array}{c}\text { Trajectory } 1 \text { vs } 2 \\
(n=388)\end{array}$ & $\begin{array}{c}\text { Trajectory } 3 \text { vs } 2 \\
(n=339)\end{array}$ & $\begin{array}{c}\text { Trajectory } 4 \text { vs } 2 \\
(n=351)\end{array}$ & $\begin{array}{c}\text { Trajectory } 5 \text { vs } 2 \\
(n=339)\end{array}$ & $\begin{array}{c}\text { Trajectory } 4 \text { vs } 3 \\
(n=134)\end{array}$ & $\begin{array}{c}\text { Trajectory } 4 \text { vs } 5 \\
(n=143)\end{array}$ \\
\hline Variable & OR $(95 \%$ CI $)$ & OR $(95 \%$ CI $)$ & OR $(95 \%$ CI $)$ & OR $(95 \%$ CI $)$ & OR $(95 \%$ CI $)$ & OR $(95 \% \mathrm{CI})$ \\
\hline \multicolumn{7}{|l|}{ Age } \\
\hline 55 and Under & Ref & Ref & Ref & Ref & Ref & Ref \\
\hline $56-60$ & $0.65(0.32-1.34)$ & $0.76(0.31-1.86)$ & $1.48(0.59-3.74)$ & $0.39(0.13-1.16)$ & $3.11(0.84-11.56)$ & $4.92(1.12-21.58) *$ \\
\hline $61-65$ & $1.06(0.50-2.22)$ & $0.56(0.22-1.45)$ & $1.05(0.40-2.77)$ & $0.38(0.12-1.16)$ & $2.21(0.57-8.55)$ & $4.37(1.00-19.05) *$ \\
\hline $66-70$ & $0.30(0.07-1.28)$ & $1.10(0.26-4.6)$ & $0.31(0.09-1.14)$ & $0.08(0.02-0.34) *$ & $0.38(0.06-2.60)$ & $8.23(1.31-51.87) *$ \\
\hline 71 and Older & $0.07(0.01-0.89)^{*}$ & $0.29(0.02-3.53)$ & $0.25(0.04-1.46)$ & $0.14(0.02-0.85)^{*}$ & $1.02(0.05-20.03)$ & $1.52(0.15-15.64)$ \\
\hline \multicolumn{7}{|l|}{ Gender } \\
\hline Male & Ref & Ref & Ref & Ref & Ref & Ref \\
\hline Female & $0.50(0.29-0.88)^{*}$ & $1.00(0.52-1.92)$ & $1.88(1.02-3.44) *$ & $2.31(1.04-5.14) *$ & $2.20(0.89-5.44)$ & $0.70(0.28-1.78)$ \\
\hline \multicolumn{7}{|l|}{ Race/Ethnicity } \\
\hline White & Ref & Ref & Ref & Ref & Ref & Ref \\
\hline Black & $0.57(0.20-1.59)$ & $0.66(0.26-1.67)$ & $0.57(0.23-1.39)$ & $0.77(0.29-2.06)$ & $0.94(0.27-3.27)$ & $0.67(0.22-1.98)$ \\
\hline Hispanic & $0.77(0.27-2.17)$ & $0.45(0.12-1.72)$ & $1.69(0.59-4.78)$ & $0.98(0.25-3.87)$ & $5.21(0.91-29.74)$ & $1.99(0.47-8.52)$ \\
\hline Other & $1.15(0.10-13.86)$ & $\mathrm{NE}$ & $0.87(0.04-19.91)$ & $7.58(0.32-181.53)$ & $\mathrm{NE}$ & $\mathrm{NE}$ \\
\hline $\begin{array}{l}\text { Education } \\
\text { Bachelor's Degree } \\
\quad \text { or Higher }\end{array}$ & Ref & Ref & Ref & Ref & Ref & Ref \\
\hline Some College & $0.78(0.35-1.73)$ & $2.74(0.80-9.42)$ & $1.22(0.40-3.75)$ & $2.01(0.55-7.35)$ & $0.45(0.08-2.46)$ & $0.52(0.10-2.59)$ \\
\hline HS Diploma & $0.78(0.37-1.62)$ & $2.16(0.66-7.07)$ & $2.01(0.77-5.24)$ & $1.15(0.36-3.67)$ & $1.64(0.34-8.06)$ & $0.81(0.18-3.72)$ \\
\hline Less than HS & $0.43(0.16-1.13)$ & $3.85(1.11-13.34)^{*}$ & $1.43(0.50-4.12)$ & $1.23(0.36-4.22)$ & $0.77(0.15-3.98)$ & $1.37(0.31-6.07)$ \\
\hline
\end{tabular}


Table 4-16. Continued

\begin{tabular}{|c|c|c|c|c|c|c|}
\hline & $\begin{array}{c}\begin{array}{c}\text { Trajectory } 1 \text { vs } 2 \\
(n=388)\end{array} \\
\end{array}$ & $\begin{array}{c}\text { Trajectory } 3 \text { vs } 2 \\
(n=339)\end{array}$ & $\begin{array}{c}\text { Trajectory } 4 \text { vs } 2 \\
(n=351)\end{array}$ & $\begin{array}{c}\text { Trajectory } 5 \text { vs } 2 \\
(n=339)\end{array}$ & $\begin{array}{c}\text { Trajectory } 4 \text { vs } 3 \\
(n=134)\end{array}$ & $\begin{array}{c}\text { Trajectory } 4 \text { vs } 5 \\
(n=143)\end{array}$ \\
\hline Variable & OR $(95 \%$ CI) & OR $(95 \%$ CI) & OR $(95 \%$ CI) & OR (95\% CI) & OR (95\% CI) & OR (95\% CI) \\
\hline \multicolumn{7}{|l|}{ Coupled Household } \\
\hline Coupled & Ref & Ref & Ref & Ref & Ref & Ref \\
\hline Not Coupled & $1.45(0.71-2.99)$ & $1.55(0.73-3.28)$ & $0.57(0.25-1.26)$ & $1.72(0.75-3.92)$ & $0.35(0.12-1.02)$ & $0.32(0.12-0.90)^{*}$ \\
\hline \multicolumn{7}{|l|}{ Work Status } \\
\hline Currently Wkg. & Ref & Ref & Ref & Ref & Ref & Ref \\
\hline Not Working & $6.10(2.69-13.80)^{*}$ & $1.04(0.51-2.12)$ & $0.53(0.27-1.05)$ & $0.42(0.19-0.96)^{*}$ & $0.34(0.11-1.05)$ & $0.86(0.28-2.63)$ \\
\hline \multicolumn{7}{|l|}{$\begin{array}{l}\text { Household Assets } \\
\text { Highest }\end{array}$} \\
\hline Quintile & Ref & Ref & Ref & Ref & Ref & Ref \\
\hline High Quintile & $1.16(0.54-2.53)$ & $1.53(0.53-4.48)$ & $2.40(0.93-6.20)$ & $1.69(0.50-5.75)$ & $1.71(0.38-7.78)$ & $1.31(0.28-6.11)$ \\
\hline Med. Quintile & $0.72(0.32-1.60)$ & $3.01(1.10-8.21)^{*}$ & $2.13(0.80-5.70)$ & $0.55(0.13-2.29)$ & $0.69(0.16-3.03)$ & $2.30(0.41-12.73)$ \\
\hline $\begin{array}{l}\text { Low Quintile } \\
\text { Lowest }\end{array}$ & $0.85(0.36-1.99)$ & $1.69(0.55-5.14)$ & $2.63(0.94-7.38)$ & $3.15(0.93-10.61)$ & $1.77(0.37-8.52)$ & $0.58(0.13-2.57)$ \\
\hline Quintile & $0.60(0.23-1.58)$ & $1.81(0.56-5.88)$ & $3.90(1.33-11.42)^{*}$ & $3.35(0.97-11.61)$ & $1.29(0.26-6.55)$ & $0.89(0.20-3.93)$ \\
\hline \multicolumn{7}{|l|}{$\begin{array}{l}\text { Health Conditions } \\
\text { No Health }\end{array}$} \\
\hline 1 Condition & $0.25(0.14-0.45)^{*}$ & $2.59(0.95-7.04)$ & $4.00(1.37-11.67)^{*}$ & $3.57(0.85-15.07)$ & $2.39(0.50-11.33)$ & $1.39(0.18-11.02)$ \\
\hline $\begin{array}{l}2 \text { Conditions } \\
3 \text { or More }\end{array}$ & $0.17(0.08-0.37)^{*}$ & $3.47(1.21-9.98)^{*}$ & $4.20(1.40-12.61) *$ & $5.16(1.22-21.80)^{*}$ & $2.52(0.51-12.50)$ & $0.42(0.05-3.23)$ \\
\hline Conditions & $0.05(0.01-0.23)^{*}$ & $4.93(1.58-15.31)^{*}$ & $6.77(2.12-21.61)^{*}$ & $18.50(4.39-77.95) *$ & $3.65(0.64-20.74)$ & $0.32(0.04-2.54)$ \\
\hline
\end{tabular}


Table 4-16. Continued

\begin{tabular}{|c|c|c|c|c|c|c|}
\hline & $\begin{array}{l}\text { Trajectory } 1 \text { vs } \\
2(n=388)\end{array}$ & $\begin{array}{c}\text { Trajectory } 3 \text { vs } 2 \\
(n=339)\end{array}$ & $\begin{array}{c}\text { Trajectory } 4 \text { vs } 2 \\
(n=351)\end{array}$ & $\begin{array}{c}\text { Trajectory } 5 \text { vs } 2 \\
(n=339\end{array}$ & $\begin{array}{c}\text { Trajectory } 4 \text { vs } \\
3(n=134)\end{array}$ & $\begin{array}{c}\text { Trajectory } 4 \text { vs } \\
5(n=143)\end{array}$ \\
\hline \multicolumn{7}{|l|}{ Insurance Status } \\
\hline Public Ins. & $3.25(0.95-11.17)$ & $0.84(0.24-2.97)$ & $2.95(1.13-7.66)^{*}$ & $5.64(1.95-16.27)^{*}$ & $1.90(0.42-8.67)$ & $0.50(0.15-1.74)$ \\
\hline No Insurance & $2.08(0.73-5.89)$ & $3.04(1.23-7.53)^{*}$ & $0.69(0.20-2.39)$ & $1.30(0.36-4.68)$ & $0.26(0.06-1.14)$ & $1.04(0.18-5.98)$ \\
\hline
\end{tabular}


and more likely to have 1 or more health conditions. Individuals in Trajectory 5 were slightly more likely to be younger, less likely to be male, more likely to be in the lowest household asset quintile, more likely to have 2 or more health conditions, and more likely to be publicly insured. (Table 4-17)

Similar to the bivariate regression analyses, this analysis also demonstrated that women and individuals with a greater number of health conditions were more likely to belong to trajectory subgroups with poorer outcomes. Additionally, this analysis found that belonging to the lowest household assets quintile was also associated with trajectories that experience poor outcomes. Insurance status was also associated with functional outcomes, with both public insurance and no insurance being identified as predictors of trajectories with poor outcomes.

\section{Part II. Mediation Analysis}

For Part II of the study, the objective is to assess the mechanism by which health insurance status goes on to effect functional status after injury. Specifically, the aim of this section of the study is to determine whether or not medical care use acts as a mediator between respondents' insurance status and their functional outcome.

Mediation was assessed using a four-step process described by Baron and Kenny (1986). Step 1 of the mediation analysis examined whether insurance status was significantly associated with functional status. This step reveals the total effect of insurance status on functional status, which may or may not be significant. Step 2 determined whether insurance status is significantly associated with the mediating variables, doctor visits, prescription drug use, and outpatient surgery. Step 3 determined whether the mediators were significantly associated with functional status while controlling for insurance status. Step 4 examined the effect of insurance status on functional status while controlling for medical care use.

\section{Step 1: Establishing the Relationship between Insurance Status and}

Functional Status. In Model 1, functional limitations were modeled as a function of age, gender, race/ethnicity, educational attainment, coupled household status, work status, household assets, number of health conditions, injury status, insurance status, and time. This analysis found that public insurance was associated with greater functional limitations. Additionally, this model also found that age, gender, education, coupled household status, work status, household assets, number of health conditions, and reinjury were also predictive of functional status. Being female, having less than a high school education, not living in coupled households, not working, having a greater number of health conditions, and being injured were associated with more functional limitations. A greater household asset value was associated with fewer functional limitations (estimate $=-3.94 \mathrm{E}-7$ ). Time was also significant in this model and functional limitations scores were greater during the second wave of the study (peri-injury period) compared to the first wave (pre-injury period). 
Table 4-17. Multinomial Logistic Regression Analysis of Characteristics that Best Discriminate Functional Status Trajectories 1, 3, 4, and 5 Compared to 2

\begin{tabular}{|c|c|c|c|c|c|c|}
\hline Variable & Level & Beta & $\mathbf{P}$ & OR & $\begin{array}{l}95 \% \text { CI } \\
\text { Lower }\end{array}$ & $\begin{array}{l}95 \% \text { CI } \\
\text { Lower }\end{array}$ \\
\hline \multicolumn{7}{|c|}{ Trajectory 1} \\
\hline Age & & -0.025 & 0.279 & 0.98 & 0.93 & 1.02 \\
\hline Male & & 0.699 & 0.011 & 2.01 & 1.18 & 3.44 \\
\hline \multirow[t]{4}{*}{ Race/Ethnicity } & White & Ref & & & & \\
\hline & Black & -0.681 & 0.188 & 0.51 & 0.18 & 1.39 \\
\hline & Hispanic & -0.318 & 0.524 & 0.73 & 0.27 & 1.93 \\
\hline & Other & 0.054 & 0.966 & 1.06 & 0.09 & 12.65 \\
\hline \multirow[t]{4}{*}{ Education } & Bachelor's Deg. or Higher & Ref & & & & \\
\hline & Some College & -0.217 & 0.58 & 0.81 & 0.37 & 1.74 \\
\hline & HS Diploma & -0.241 & 0.503 & 0.79 & 0.39 & 1.59 \\
\hline & Less than HS & -0.677 & 0.144 & 0.51 & 0.21 & 1.26 \\
\hline Coupled Household & & -0.44 & 0.204 & 0.64 & 0.33 & 1.27 \\
\hline Currently Working & & 1.716 & $<0.001$ & 5.56 & 2.49 & 12.42 \\
\hline \multirow[t]{5}{*}{ Household Assets } & Highest Quintile & Ref & & & & \\
\hline & High Quintile & 0.256 & 0.505 & 1.29 & 0.61 & 2.75 \\
\hline & Medium Quintile & -0.211 & 0.597 & 0.81 & 0.37 & 1.77 \\
\hline & Low Quintile & -0.242 & 0.56 & 0.79 & 0.35 & 1.77 \\
\hline & Lowest Quintile & -0.447 & 0.346 & 0.64 & 0.25 & 1.62 \\
\hline \multirow[t]{2}{*}{$\begin{array}{l}\text { Number of Health } \\
\text { Conditions }\end{array}$} & No Health Conditions & Ref & & & & \\
\hline & 1 Condition & -1.342 & $<0.001$ & 0.26 & 0.15 & 0.46 \\
\hline
\end{tabular}


Table 4-17. Continued

\begin{tabular}{|c|c|c|c|c|c|c|}
\hline Variable & Level & Beta & $\mathbf{P}$ & OR & $\begin{array}{c}\text { 95\% CI } \\
\text { Lower }\end{array}$ & $\begin{array}{c}95 \% \mathrm{CI} \\
\text { Upper }\end{array}$ \\
\hline \multicolumn{7}{|c|}{ Trajectory 1 (Continued) } \\
\hline Number of Health & & & & & & \\
\hline \multirow[t]{2}{*}{ Conditions (Cont'd.) } & 2 Conditions & -1.8 & $<0.001$ & 0.17 & 0.08 & 0.36 \\
\hline & 3 or More Conditions & -2.762 & $<0.001$ & 0.06 & 0.01 & 0.29 \\
\hline \multirow[t]{3}{*}{ Insurance Status } & Private Insurance & Ref & & & & \\
\hline & Public Insurance & 0.463 & 0.269 & 1.59 & 0.7 & 3.61 \\
\hline & No Insurance & 0.631 & 0.203 & 1.88 & 0.71 & 4.97 \\
\hline \multicolumn{7}{|c|}{ Trajectory 3} \\
\hline Age & & -0.02 & 0.498 & 0.98 & 0.93 & 1.04 \\
\hline Male & & -0.037 & 0.908 & 0.96 & 0.51 & 1.81 \\
\hline \multirow{4}{*}{ Race/Ethnicity } & White & Ref & & & & \\
\hline & Black & -0.359 & 0.42 & 0.7 & 0.29 & 1.67 \\
\hline & Hispanic & -0.892 & 0.185 & 0.41 & 0.11 & 1.53 \\
\hline & Other & $\mathrm{NE}$ & & & & \\
\hline \multirow[t]{4}{*}{ Education } & Bachelor's Deg. or Higher & Ref & & & & \\
\hline & Some College & 1.127 & 0.067 & 3.09 & 0.93 & 10.3 \\
\hline & HS Diploma & 0.74 & 0.211 & 2.1 & 0.66 & 6.69 \\
\hline & Less than HS & 1.289 & 0.037 & 3.63 & 1.08 & 12.16 \\
\hline Coupled Household & & -0.422 & 0.251 & 0.66 & 0.32 & 1.35 \\
\hline Currently Working & & 0.171 & 0.631 & 1.19 & 0.59 & 2.38 \\
\hline
\end{tabular}


Table 4-17. Continued

\begin{tabular}{|c|c|c|c|c|c|c|}
\hline Variable & Level & Beta & $\mathbf{P}$ & OR & $\begin{array}{c}95 \% \text { CI } \\
\text { Lower }\end{array}$ & $\begin{array}{c}\text { 95\% CI } \\
\text { Upper }\end{array}$ \\
\hline \multicolumn{7}{|c|}{ Trajectory 3 Continued } \\
\hline \multirow[t]{5}{*}{ Household Assets } & Highest Quintile & Ref & & & & \\
\hline & High Quintile & 0.495 & 0.354 & 1.64 & 0.58 & 4.67 \\
\hline & Medium Quintile & 0.994 & 0.048 & 2.7 & 1.01 & 7.25 \\
\hline & Low Quintile & 0.489 & 0.369 & 1.63 & 0.56 & 4.75 \\
\hline & Lowest Quintile & 0.579 & 0.308 & 1.79 & 0.59 & 5.44 \\
\hline \multirow{4}{*}{$\begin{array}{l}\text { Number of Health } \\
\text { Conditions }\end{array}$} & No Health Conditions & Ref & & & & \\
\hline & 1 Condition & 0.959 & 0.055 & 2.61 & 0.98 & 6.95 \\
\hline & 2 Conditions & 1.231 & 0.018 & 3.42 & 1.23 & 9.52 \\
\hline & 3 or More Conditions & 1.523 & 0.006 & 4.58 & 1.54 & 13.66 \\
\hline \multirow[t]{3}{*}{ Insurance Status } & Private Insurance & Ref & & & & \\
\hline & Public Insurance & 0.208 & 0.649 & 1.23 & 0.5 & 3.01 \\
\hline & No Insurance & 1.069 & 0.017 & 2.91 & 1.21 & 7.01 \\
\hline \multicolumn{7}{|c|}{ Trajectory 4} \\
\hline Age & & -0.029 & 0.273 & 0.97 & 0.92 & 1.02 \\
\hline Male & & -0.556 & 0.064 & 0.57 & 0.32 & 1.03 \\
\hline \multirow[t]{4}{*}{ Race/Ethnicity } & White & Ref & & & & \\
\hline & Black & -0.29 & 0.487 & 0.75 & 0.33 & 1.7 \\
\hline & Hispanic & 0.238 & 0.639 & 1.27 & 0.47 & 3.43 \\
\hline & Other & 0.575 & 0.656 & 1.78 & 0.14 & 22.29 \\
\hline
\end{tabular}


Table 4-17. Continued

\begin{tabular}{|c|c|c|c|c|c|c|}
\hline Variable & Level & Beta & $\mathbf{p}$ & OR & $\begin{array}{l}\text { 95\% CI } \\
\text { Lower }\end{array}$ & $\begin{array}{l}\text { 95\% CI } \\
\text { Higher }\end{array}$ \\
\hline \multicolumn{7}{|c|}{ Trajectory 4 Continued } \\
\hline \multirow[t]{4}{*}{ Education } & Bachelor's Deg.or Higher & Ref & & & & \\
\hline & Some College & 0.353 & 0.522 & 1.42 & 0.48 & 4.19 \\
\hline & HS Diploma & 0.838 & 0.073 & 2.31 & 0.92 & 5.78 \\
\hline & Less than HS & 0.667 & 0.197 & 1.95 & 0.71 & 5.37 \\
\hline Coupled Household & & 0.306 & 0.411 & 1.36 & 0.66 & 2.82 \\
\hline Currently Working & & -0.465 & 0.154 & 0.63 & 0.33 & 1.19 \\
\hline \multirow[t]{5}{*}{ Household Assets } & Highest Quintile & Ref & & & & \\
\hline & High Quintile & 0.789 & 0.097 & 2.2 & 0.87 & 5.59 \\
\hline & Medium Quintile & 0.689 & 0.161 & 1.99 & 0.76 & 5.22 \\
\hline & Low Quintile & 0.774 & 0.128 & 2.17 & 0.8 & 5.88 \\
\hline & Lowest Quintile & 1.072 & 0.037 & 2.92 & 1.07 & 8.01 \\
\hline \multirow[t]{4}{*}{$\begin{array}{l}\text { Number of Health } \\
\text { Conditions }\end{array}$} & No Health Conditions & Ref & & & & \\
\hline & 1 Condition & 1.288 & 0.014 & 3.62 & 1.3 & 10.14 \\
\hline & 2 Conditions & 1.365 & 0.012 & 3.92 & 1.35 & 11.39 \\
\hline & 3 or More Conditions & 2.075 & $<0.001$ & 7.97 & 2.63 & 24.12 \\
\hline \multirow[t]{3}{*}{ Insurance Status } & Private Insurance & Ref & & & & \\
\hline & Public Insurance & 0.442 & 0.28 & 1.56 & 0.7 & 3.47 \\
\hline & No Insurance & -0.269 & 0.656 & 0.76 & 0.23 & 2.49 \\
\hline \multicolumn{7}{|c|}{ Trajectory 5} \\
\hline Age & & -0.06 & 0.041 & 0.94 & 0.89 & 1 \\
\hline Male & & -0.82 & 0.024 & 0.44 & 0.22 & 0.9 \\
\hline
\end{tabular}


Table 4-17. Continued

\begin{tabular}{|c|c|c|c|c|c|c|}
\hline Variable & Level & Beta & $\mathbf{p}$ & OR & $\begin{array}{c}95 \% \text { CI } \\
\text { Lower }\end{array}$ & $\begin{array}{l}95 \% \text { CI } \\
\text { Higher }\end{array}$ \\
\hline \multicolumn{7}{|c|}{ Trajectory 5 Continued } \\
\hline \multirow[t]{4}{*}{ Race/Ethnicity } & White & Ref & & & & \\
\hline & Black & -0.051 & 0.908 & 0.95 & 0.4 & 2.26 \\
\hline & Hispanic & 0.127 & 0.834 & 1.14 & 0.35 & 3.71 \\
\hline & Other & 0.272 & 0.872 & 1.31 & 0.05 & 36.33 \\
\hline \multirow[t]{4}{*}{ Education } & $\begin{array}{l}\text { Bachelor's Degree or } \\
\text { Higher }\end{array}$ & Ref & & & & \\
\hline & Some College & 1.006 & 0.098 & 2.73 & 0.83 & 8.98 \\
\hline & HS Diploma & 0.454 & 0.411 & 1.57 & 0.53 & 4.65 \\
\hline & Less than HS & 0.697 & 0.226 & 2.01 & 0.65 & 6.2 \\
\hline Coupled Household & & -0.496 & 0.188 & 0.61 & 0.29 & 1.28 \\
\hline Currently Working & & -0.71 & 0.068 & 0.49 & 0.23 & 1.06 \\
\hline \multirow[t]{5}{*}{ Household Assets } & Highest Quintile & Ref & & & & \\
\hline & High Quintile & 0.592 & 0.315 & 1.81 & 0.57 & 5.74 \\
\hline & Medium Quintile & -0.23 & 0.731 & 0.8 & 0.22 & 2.94 \\
\hline & Low Quintile & 1.089 & 0.06 & 2.97 & 0.96 & 9.25 \\
\hline & Lowest Quintile & 1.294 & 0.027 & 3.65 & 1.16 & 11.5 \\
\hline \multirow[t]{4}{*}{ No. of Health Conditions } & No Health Conditions & Ref & & & & \\
\hline & 1 Condition & 1.048 & 0.136 & 2.85 & 0.72 & 11.29 \\
\hline & 2 Conditions & 1.637 & 0.019 & 5.14 & 1.31 & 20.15 \\
\hline & 3 or More Conditions & 2.708 & $<0.001$ & 15 & 3.86 & 58.28 \\
\hline \multirow[t]{3}{*}{ Insurance Status } & Private Insurance & Ref & & & & \\
\hline & Public Insurance & 1.147 & 0.012 & 3.15 & 1.29 & 7.71 \\
\hline & No Insurance & 0.277 & 0.65 & 1.32 & 0.4 & 4.36 \\
\hline
\end{tabular}


Table 4-18 displays unstandardized coefficient estimates and $p$ values from Model 1. It is important to note that coefficient estimates cannot be compared across models for data displayed in Tables 4-18 through 4-22. Comparable coefficient estimates are reported in the following section.

Step 2: Establishing the Relationship between Insurance Status and Medical Care Use. Models 2, 3, and 4 model each individual mediator as a function of insurance status along with all covariates contained in Model 1.

Doctor Visits. In Model 2, number of doctor visits were modeled as a function of age, gender, race/ethnicity, educational attainment, coupled household status, work status, household assets, number of health conditions, injury status, insurance status, and time. (Table 4-19)

This analysis found that public insurance was associated with a greater number of doctor visits. Additionally, this model also found education, work status, household assets, and number of health conditions were also significant predictors of doctor visits. Not finishing high school, having a greater value of household assets (estimate $=-4.26 \mathrm{E}$ 7), and having only a high school diploma were associated with a decreased number of doctor visits. Additionally, not working and having 1 or more health conditions were associated with increases in doctor visits. Age, gender, race/ethnicity, coupled household, and time were not significant in Model 2.

Prescription Drug Use. In Model 3, whether or not an individual used prescription medication was modeled as a function of age, gender, race/ethnicity, educational attainment, coupled household status, work status, household assets, number of health conditions, injury status, insurance status, and time. (Table 4-20)

This analysis found that being uninsured was associated with not using prescription drugs. Additionally, this model found that age, gender, education, coupled household, household assets, and number of health conditions were significant predictors of prescription drug use. Older age groups, females, and having 1 or more health conditions were significant positive predictors of medication use. Individuals without bachelor's degrees, people not living with a spouse, and those with fewer household assets (estimate $=-1.01 \mathrm{E}-7$ ) were less likely to use prescription drugs. Race/ethnicity, work status, injury status, and time were not significant in Model 3.

Outpatient Surgery. In Model 4, whether or not an individual underwent outpatient surgery was modeled as a function of age, gender, race/ethnicity, educational attainment, coupled household status, work status, household assets, number of health conditions, injury status, insurance status, and time. (Table 4-21)

This analysis found that having no insurance reduced the likelihood of having surgery. Additionally, this model found that number of health conditions was significantly associated with having surgery. A larger value of household assets was also 
Table 4-18. $\quad$ Model 1 Effect Estimates (Unstandardized)

\begin{tabular}{|c|c|c|}
\hline \multirow[b]{2}{*}{ Variable } & \multicolumn{2}{|c|}{$\begin{array}{c}\text { Dependent Variable = } \\
\text { Functional Limitations } \\
\text { Score } \\
\end{array}$} \\
\hline & Estimate & p Value \\
\hline \multicolumn{3}{|l|}{ Age } \\
\hline 55 and Under & Ref & \\
\hline $56-60$ & -0.12 & 0.930 \\
\hline $61-65$ & -1.34 & 0.392 \\
\hline $66-70$ & -4.85 & $0.024 *$ \\
\hline 71 and Older & -1.80 & 0.419 \\
\hline \multicolumn{3}{|l|}{ Gender } \\
\hline Male & Ref & \\
\hline Female & 5.25 & $0.003 *$ \\
\hline \multicolumn{3}{|l|}{ Race/Ethnicity } \\
\hline White & Ref & \\
\hline Black & 1.01 & 0.633 \\
\hline Hispanic & 0.05 & 0.984 \\
\hline Other & 0.31 & 0.964 \\
\hline \multicolumn{3}{|l|}{ Education } \\
\hline \multicolumn{3}{|l|}{ Bachelor's Degree or } \\
\hline Some College & 3.11 & 0.199 \\
\hline HS Diploma & 3.48 & 0.115 \\
\hline Less than HS & 8.27 & $0.004 *$ \\
\hline \multicolumn{3}{|l|}{ Coupled Household } \\
\hline Coupled & Ref & \\
\hline Not Coupled & 2.99 & $0.024 *$ \\
\hline \multicolumn{3}{|l|}{ Work Status } \\
\hline Currently Working & Ref & \\
\hline Not Working & 4.37 & $<0.001 *$ \\
\hline Household Assets & 0.00 & $<0.001 *$ \\
\hline \multicolumn{3}{|l|}{ Number of Health } \\
\hline \multicolumn{3}{|l|}{ Conditions } \\
\hline No Health Conditions & Ref & \\
\hline 1 Condition & 6.00 & $<0.001 *$ \\
\hline 2 Conditions & 10.93 & $<0.001 *$ \\
\hline 3 or More Conditions & 15.84 & $<0.001 *$ \\
\hline
\end{tabular}


Table 4-18. Continued

\begin{tabular}{|c|c|c|}
\hline \multirow[b]{2}{*}{ Variable } & \multicolumn{2}{|c|}{$\begin{array}{c}\text { Dependent Variable }= \\
\text { Functional Limitations } \\
\text { Score }\end{array}$} \\
\hline & Estimate & p Value \\
\hline \multicolumn{3}{|l|}{ Injury } \\
\hline No Injury & Ref & \\
\hline Injury & 2.92 & $0.004 *$ \\
\hline \multicolumn{3}{|l|}{ Insurance Status } \\
\hline Private Insurance & Ref & \\
\hline Public Insurance & 2.98 & $0.013 *$ \\
\hline No Insurance & 1.06 & 0.441 \\
\hline \multicolumn{3}{|l|}{ Survey Wave } \\
\hline Wave 1 (1998) & Ref & \\
\hline Wave 2 (2000) & 3.58 & $<0.001 *$ \\
\hline Wave 3 (2002) & 0.60 & 0.489 \\
\hline Wave 4 (2004) & 0.73 & 0.459 \\
\hline Wave 5 (2006) & 1.56 & 0.174 \\
\hline Wave 6 (2008) & 1.18 & 0.370 \\
\hline
\end{tabular}


Table 4-19. $\quad$ Model 2 Effect Estimates (Unstandardized)

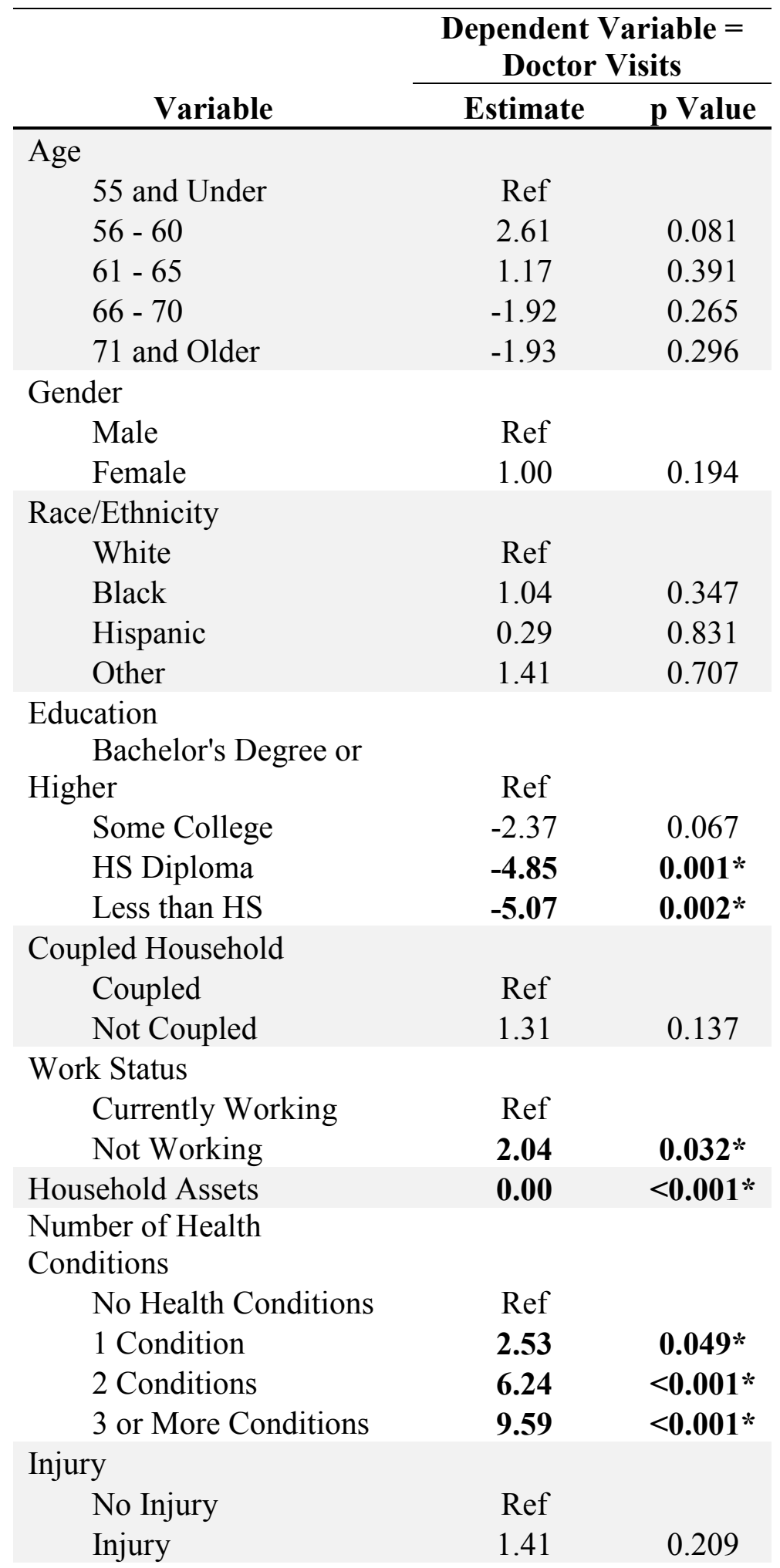


Table 4-19. Continued

\begin{tabular}{ccc}
\hline & \multicolumn{2}{c}{$\begin{array}{c}\text { Dependent Variable }= \\
\text { Doctor Visits }\end{array}$} \\
\cline { 2 - 3 } Variable & Estimate & p Value \\
\cline { 2 - 3 } Insurance Status & Ref & \\
Private Insurance & $\mathbf{2 . 6 9}$ & $\mathbf{0 . 0 4 7}$ \\
Public Insurance & -2.27 & 0.143 \\
No Insurance & & \\
Survey Wave & Ref & \\
Wave 1 (1998) & 0.01 & 0.992 \\
Wave 2 (2000) & -0.71 & 0.536 \\
Wave 3 (2002) & -1.11 & 0.343 \\
Wave 4 (2004) & -0.67 & 0.582 \\
Wave 5 (2006) & -2.50 & 0.053 \\
Wave 6 (2008) &
\end{tabular}

$* \mathrm{p}<0.05$ 
Table 4-20. Model 3 Effect Estimates (Unstandardized)

\begin{tabular}{|c|c|c|}
\hline \multirow[b]{2}{*}{ Variable } & \multicolumn{2}{|c|}{$\begin{array}{c}\text { Dependent } \\
\text { Variable }=\text { RX }\end{array}$} \\
\hline & Estimate & p Value \\
\hline \multicolumn{3}{|l|}{ Age } \\
\hline 55 and Under & Ref & \\
\hline $56-60$ & 0.63 & $0.005^{*}$ \\
\hline $61-65$ & 0.56 & $0.010 *$ \\
\hline $66-70$ & 0.51 & 0.059 \\
\hline 71 and Older & 0.79 & $0.016 *$ \\
\hline \multicolumn{3}{|l|}{ Gender } \\
\hline Male & Ref & \\
\hline Female & 1.06 & $<0.001 *$ \\
\hline \multicolumn{3}{|l|}{ Race/Ethnicity } \\
\hline White & Ref & \\
\hline Black & 0.10 & 0.576 \\
\hline Hispanic & -0.04 & 0.833 \\
\hline Other & 0.56 & 0.325 \\
\hline \multicolumn{3}{|l|}{ Education } \\
\hline \multicolumn{3}{|l|}{ Bachelor's Degree or } \\
\hline Higher & Ref & \\
\hline Some College & -0.56 & $0.009 *$ \\
\hline HS Diploma & -0.60 & $0.004 *$ \\
\hline Less than HS & -0.53 & $0.016 *$ \\
\hline \multicolumn{3}{|l|}{ Coupled Household } \\
\hline Coupled & Ref & \\
\hline Not Coupled & -0.46 & $0.005^{*}$ \\
\hline \multicolumn{3}{|l|}{ Work Status } \\
\hline Currently Working & Ref & \\
\hline Not Working & 0.11 & 0.363 \\
\hline Household Assets & $\mathbf{0 . 0 0}$ & $<0.001 *$ \\
\hline \multicolumn{3}{|l|}{ Number of Health Conditions } \\
\hline No Health Conditions & Ref & \\
\hline 1 Condition & 1.59 & $<0.001 *$ \\
\hline 2 Conditions & 2.40 & $<0.001 *$ \\
\hline 3 or More Conditions & 3.97 & $<0.001 *$ \\
\hline \multicolumn{3}{|l|}{ Injury } \\
\hline No Injury & Ref & \\
\hline Injury & 0.34 & 0.092 \\
\hline
\end{tabular}


Table 4-20. Continued

\begin{tabular}{ccc}
\hline & \multicolumn{2}{c}{$\begin{array}{c}\text { Dependent } \\
\text { Variable }=\mathbf{R X}\end{array}$} \\
\cline { 2 - 3 } & Estimate & $\mathbf{p ~ V a l u e}$ \\
\cline { 2 - 3 } Insurance Status & Ref & \\
Private Insurance & 0.28 & 0.165 \\
Public Insurance & $\mathbf{- 1 . 0 8}$ & $<\mathbf{0 . 0 0 1 *}$ \\
No Insurance & & \\
Survey Wave & Ref & \\
Wave 1 (1998) & 0.03 & 0.887 \\
Wave 2 (2000) & -0.03 & 0.847 \\
Wave 3 (2002) & -0.10 & 0.567 \\
Wave 4 (2004) & -0.04 & 0.842 \\
Wave 5 (2006) & -0.16 & 0.425 \\
Wave 6 (2008) &
\end{tabular}

$* \mathrm{p}<0.05$ 
Table 4-21. Model 4 Effect Estimates (Unstandardized)

\begin{tabular}{|c|c|c|}
\hline \multirow[b]{2}{*}{ Variable } & \multicolumn{2}{|c|}{$\begin{array}{c}\text { Dependent } \\
\text { Variable = } \\
\text { Outpatient } \\
\text { Surgery }\end{array}$} \\
\hline & Estimate & p Value \\
\hline \multicolumn{3}{|l|}{ Age } \\
\hline 55 and Under & Ref & \\
\hline $56-60$ & -0.32 & 0.083 \\
\hline $61-65$ & -0.30 & 0.101 \\
\hline $66-70$ & -0.25 & 0.240 \\
\hline 71 and Older & -0.18 & 0.422 \\
\hline \multicolumn{3}{|l|}{ Gender } \\
\hline Male & Ref & \\
\hline Female & 0.05 & 0.578 \\
\hline \multicolumn{3}{|l|}{ Race/Ethnicity } \\
\hline White & Ref & \\
\hline Black & -0.22 & 0.136 \\
\hline Hispanic & 0.02 & 0.904 \\
\hline Other & 0.22 & 0.611 \\
\hline \multicolumn{3}{|l|}{ Education } \\
\hline \multicolumn{3}{|l|}{ Bachelor's Degree or } \\
\hline Higher & Ref & \\
\hline Some College & 0.00 & 0.982 \\
\hline HS Diploma & -0.33 & $0.021 *$ \\
\hline Less than HS & -0.54 & $0.003 *$ \\
\hline \multicolumn{3}{|l|}{ Coupled Household } \\
\hline Coupled & Ref & \\
\hline Not Coupled & -0.23 & $0.047 *$ \\
\hline \multicolumn{3}{|l|}{ Work Status } \\
\hline Currently Working & Ref & \\
\hline Not Working & 0.06 & 0.553 \\
\hline Household Assets & 0.00 & $<0.001 *$ \\
\hline \multicolumn{3}{|l|}{ Number of Health } \\
\hline \multicolumn{3}{|l|}{ Conditions } \\
\hline No Health Conditions & Ref & \\
\hline 1 Condition & 0.14 & 0.356 \\
\hline 2 Conditions & 0.38 & $0.026 *$ \\
\hline 3 or More Conditions & 0.42 & $0.016 *$ \\
\hline
\end{tabular}


Table 4-21. Continued

\begin{tabular}{|c|c|c|}
\hline \multirow[b]{2}{*}{ Variable } & \multicolumn{2}{|c|}{$\begin{array}{c}\text { Dependent } \\
\text { Variable = } \\
\text { Outpatient } \\
\text { Surgery }\end{array}$} \\
\hline & Estimate & p Value \\
\hline \multicolumn{3}{|l|}{ Injury } \\
\hline No Injury & Ref & \\
\hline Injury & 0.00 & 0.995 \\
\hline \multicolumn{3}{|l|}{ Insurance Status } \\
\hline Private Insurance & Ref & \\
\hline Public Insurance & 0.22 & 0.163 \\
\hline No Insurance & -0.68 & $0.014 *$ \\
\hline \multicolumn{3}{|l|}{ Survey Wave } \\
\hline Wave 1 (1998) & Ref & \\
\hline Wave $2(2000)$ & 0.23 & 0.244 \\
\hline Wave 3 (2002) & -0.13 & 0.402 \\
\hline Wave 4 (2004) & -0.02 & 0.882 \\
\hline Wave 5 (2006) & -0.18 & 0.268 \\
\hline Wave $6(2008)$ & -0.18 & 0.265 \\
\hline
\end{tabular}

$* \mathrm{p}<0.05$ 
Table 4-22. Model 5 Effect Estimates (Unstandardized)

\begin{tabular}{|c|c|c|}
\hline \multirow[b]{2}{*}{ Variable } & \multicolumn{2}{|c|}{$\begin{array}{c}\text { Dependent Variable } \\
=\text { Functional } \\
\text { Limitations }\end{array}$} \\
\hline & Estimate & p Value \\
\hline \multicolumn{3}{|l|}{ Age } \\
\hline 55 and Under & Ref & \\
\hline $56-60$ & -0.72 & 0.598 \\
\hline $61-65$ & -1.97 & 0.240 \\
\hline $66-70$ & -5.62 & $0.027 *$ \\
\hline 71 and Older & -2.77 & 0.248 \\
\hline \multicolumn{3}{|l|}{ Gender } \\
\hline Male & Ref & \\
\hline Female & 4.77 & $0.014 *$ \\
\hline \multicolumn{3}{|l|}{ Race/Ethnicity } \\
\hline White & Ref & \\
\hline Black & 1.04 & 0.625 \\
\hline Hispanic & -0.06 & 0.982 \\
\hline Other & 0.74 & 0.914 \\
\hline \multicolumn{3}{|l|}{ Education } \\
\hline \multicolumn{3}{|l|}{ Bachelor's Degree or } \\
\hline Higher & Ref & \\
\hline Some College & 3.62 & 0.159 \\
\hline HS Diploma & 4.11 & 0.089 \\
\hline Less than HS & 8.92 & $0.009 *$ \\
\hline \multicolumn{3}{|l|}{ Coupled Household } \\
\hline Coupled & Ref & \\
\hline Not Coupled & 2.62 & 0.067 \\
\hline \multicolumn{3}{|l|}{ Work Status } \\
\hline Currently Working & Ref & \\
\hline Not Working & 4.14 & $0.003 *$ \\
\hline Household Assets & 0.00 & $<0.001 *$ \\
\hline \multicolumn{3}{|l|}{ Number of Health } \\
\hline \multicolumn{3}{|l|}{ Conditions } \\
\hline No Health Conditions & Ref & \\
\hline 1 Condition & 4.42 & $0.016 *$ \\
\hline 2 Conditions & 8.53 & $0.002 *$ \\
\hline 3 or More Conditions & 12.64 & $<0.001 *$ \\
\hline \multicolumn{3}{|l|}{ Injury } \\
\hline No Injury & Ref & \\
\hline Injury & 2.70 & $0.020 *$ \\
\hline
\end{tabular}


Table 4-22. Continued

\begin{tabular}{ccc}
\hline & \multicolumn{2}{c}{$\begin{array}{c}\text { Dependent Variable } \\
\text { = Functional } \\
\text { Limitations }\end{array}$} \\
\cline { 2 - 3 } Variable & Estimate & p Value \\
\cline { 2 - 3 } Insurance Status & Ref & \\
Private Insurance & $\mathbf{3 . 3 0}$ & $\mathbf{0 . 0 2 1} *$ \\
Public Insurance & 1.76 & 0.237 \\
No Insurance & & \\
Survey Wave & Ref & \\
Wave 1 (1998) & $\mathbf{3 . 6 1}$ & $<\mathbf{0 . 0 0 1 *}$ \\
Wave 2 (2000) & 0.67 & 0.445 \\
Wave 3 (2002) & 0.67 & 0.495 \\
Wave 4 (2004) & 1.44 & 0.207 \\
Wave 5 (2006) & 1.16 & 0.371 \\
Wave 6 (2008) & $\mathbf{0 . 1 2}$ & $<\mathbf{0 . 0 0 1 *}$ \\
& $\mathbf{3 . 2 9}$ & $\mathbf{0 . 0 2 1} *$ \\
Doctor Visits & 0.12 & 0.854 \\
\hline Uses Rx & & \\
Had Surgery & &
\end{tabular}

$* p<0.05$ 
associated with having surgery (estimate $=6.26 \mathrm{E}-9$ ). Having less education and not living with a spouse were both associated with a lower likelihood of having surgery. Age, gender, race/ethnicity, work status, injury status, and time were not significant in Model 4.

\section{Step 3: Establishing the Relationship between Medical Care Use and}

Functional Status. Model 5 examined the effects of insurance status and all medical care use variables on functional status. Age, gender, race/ethnicity, educational attainment, coupled household status, work status, household assets, number of health conditions, injury status, and time were all included as covariates in the final model. (Table 4-22) This analysis found that insurance status, doctor visits, and prescription drug use were significant predictors of functional limitations, but surgery was not. Additionally, the covariates age, gender, education, work status, household assets, number of health conditions, injury status, and time were also significant. Public insurance was associated with a greater number of functional limitations, but being uninsured was not. Both number of doctor visits and prescription drug use were also associated with a greater number of functional limitations. One age category, 66-70 was associated with fewer functional limitations compared to individuals 55 and younger. Having a greater amount of household assets was also associated with better functional status (estimate $=-3.63 \mathrm{E}$ 7). Women, respondents who did not complete high school, people who were not working, individuals with 1 or more health conditions, and those who were re-injured were more likely to have a greater number of functional limitations. Additionally, functional limitations were greater in wave 2 compared to wave 1.

\section{Comparable Coefficient and Standard Error Calculations}

Because Models 3 and 4 had binary outcomes, parameter estimates and standard errors had to be rescaled in order to create comparable estimates for the mediation analysis. Comparable estimates were calculated using the formulas described in Chapter 3. Table 4-23 presents comparable coefficient estimates and standard errors for Models $1-5$. Significant effects $(\mathrm{p}<0.05)$ are indicated by the asterisks.

\section{Relative Total, Direct, and Indirect Effects of Insurance Status}

Overall, the relative total effect of public insurance indicated that individuals with public insurance had greater functional limitations than those individuals with private insurance. The direct effect also demonstrated that this relationship held true even in the presence of mediating variables. However, the direct effect was reduced when controlling for mediating variables, indicating that the effect of public insurance on functional status is partially mediated by medical care use. The relative total indirect effect of public insurance was significant, confirming that mediation through medical care use likely takes place. Although the relative total indirect effect was significant, not all individual indirect effects were significant at the $\mathrm{p}=0.05$ level. Both prescription drug use and 
Table 4-23. Comparable Coefficient Estimates and Standard Errors

\begin{tabular}{|c|c|c|c|c|c|c|c|c|c|c|}
\hline \multirow[b]{3}{*}{ Variable } & \multirow{2}{*}{\multicolumn{2}{|c|}{$\begin{array}{l}\text { Step 1 } \\
Y=X \\
\end{array}$}} & \multicolumn{6}{|c|}{ Step 2} & \multirow{2}{*}{\multicolumn{2}{|c|}{$\begin{array}{c}\text { Steps } 3 \text { \& } 4 \\
\mathbf{Y}=\underset{M_{3}}{M_{1} M_{2}} \\
\end{array}$}} \\
\hline & & & \multicolumn{2}{|c|}{$\mathbf{M}_{1}=\mathbf{X}$} & \multicolumn{2}{|c|}{$\mathbf{M}_{2}=\mathbf{X}$} & \multicolumn{2}{|c|}{$\mathbf{M}_{3}=\mathbf{X}$} & & \\
\hline & Estimate & SE & Estimate & SE & Estimate & SE & Estimate & SE & Estimate & SE \\
\hline \multicolumn{11}{|l|}{ Insurance Status } \\
\hline No Insurance & 0.109 & 0.136 & -0.233 & 0.144 & $-0.144 * * *$ & 0.025 & $-0.092 * *$ & 0.031 & 0.117 & 0.087 \\
\hline Public & $0.617 * *$ & 0.205 & $0.554 *$ & 0.236 & 0.074 & 0.050 & 0.060 & 0.040 & $0.438 *$ & 0.132 \\
\hline Private & \multicolumn{2}{|c|}{ Reference } & \multicolumn{2}{|c|}{ Reference } & \multicolumn{2}{|c|}{ Reference } & \multicolumn{2}{|c|}{ Reference } & \multicolumn{2}{|c|}{ Reference } \\
\hline Doctor Visits $\left(\mathrm{M}_{1}\right)$ & & & & & & & & & $0.602 * * *$ & 0.084 \\
\hline \multicolumn{11}{|l|}{$\mathrm{Rx}\left(\mathrm{M}_{2}\right)$} \\
\hline Does Not Use Rx & & & & & & & & & \multicolumn{2}{|c|}{ Reference } \\
\hline Uses RX & & & & & & & & & $0.362 *$ & 0.108 \\
\hline \multicolumn{11}{|l|}{ Outpatient Surgery $\left(\mathrm{M}_{3}\right)$} \\
\hline Did Not Have Surgery & & & & & & & & & \multicolumn{2}{|c|}{ Reference } \\
\hline Had Surgery & & & & & & & & & 0.014 & 0.071 \\
\hline
\end{tabular}


insurance on functional status. Doctor visits were responsible for the majority of the indirect effect of public insurance. The estimate for doctor visits was positive, indicating that a greater number of doctor visits was associated with a greater number of functional limitations.

The relative total and direct effects of no insurance on functional status were not statistically significant; however the indirect effect of no insurance was significant. Having a significant mediated effect in the absence of a significant direct effect indicates that medical care use may act as a suppressor of the effect of no insurance on functional status. Suppression occurs when direct and indirect effects act in opposite directions on the dependent variable. In this case, having no insurance has a positive effect on functional limitations (i.e. increases limitations) and a negative effect on medical care use (i.e. decreases medical care use). The results indicate that not having insurance would be directly associated with an increase in functional limitations; however, through medical care use, not having insurance indirectly is associated with a decrease in functional limitations. The individual indirect effects provide more information on this relationship and demonstrate that not having insurance reduces medical care use, which in turn increases functional limitations. Prescription drug use was the only significant individual indirect effect. Both doctor visits and surgery were not significant mediators of the effect of not having insurance on functional status.

Table 4-24 displays all relative total, direct, and indirect effects for public insurance and no insurance in comparison to private insurance. Figure 4-4 depicts effect estimates between variables in the mediation model.

\section{Answers to Study Hypotheses}

The first study hypothesis stated "Individuals who are injured will have variation in functional status trajectories that can be classified into recovery subgroups." Using group-based trajectory modeling (GBTM), the study found that five distinct subgroups existed within the injured study sample. Therefore, the first hypothesis was accepted. Hypothesis 1 contained five additional sub-hypotheses that described characteristics of the hypothesized trajectory subgroups. Hypothesis 1a stated "Trajectory analysis will reveal a subgroup that has no functional limitations at the time of injury and remains limitations-free for the duration of the study". GBTM estimated Trajectory 1 which was characterized by members that maintain a consistently high level of functioning throughout the study. Hypothesis 1a was therefore accepted. Hypothesis $1 \mathrm{~b}$ stated "Trajectory analysis will reveal a subgroup that has significant functional limitations at the time of injury and limitations remain for the duration of the study". Trajectory 5 was characterized by consistently high scores on the functional limitations scale, satisfying hypothesis 1b. Hypothesis 1c stated "Trajectory analysis will reveal a subgroup that has functional limitations after injury and then returns to near-baseline levels of functioning throughout the post-injury period". Trajectory 2 displays similar characteristics to the subgroup described by hypothesis 1c. Therefore, hypothesis 1c was accepted. Hypothesis 1d stated "Trajectory analysis will reveal a subgroup that has functional limitations at the 
Table 4-24. Relative Total, Direct, and Indirect Effects

\begin{tabular}{|c|c|c|c|c|c|c|c|c|}
\hline \multirow[b]{2}{*}{ Variable } & \multicolumn{4}{|c|}{ Public Insurance } & \multicolumn{4}{|c|}{ No Insurance } \\
\hline & Estimate & SE & LCL & UCL & Estimate & SE & LCL & UCL \\
\hline Relative Total Effects & 0.618 & 0.205 & 0.215 & 1.021 & 0.109 & 0.136 & -0.157 & 0.376 \\
\hline Relative Direct Effects & 0.438 & 0.132 & 0.178 & 0.698 & 0.117 & 0.087 & -0.053 & $\begin{array}{c}0.287 \\
-\end{array}$ \\
\hline $\begin{array}{l}\text { Relative Total Indirect Effects } \\
\text { Relative Individual Indirect Effects }\end{array}$ & 0.361 & 0.151 & 0.064 & 0.657 & -0.194 & 0.090 & -0.370 & 0.018 \\
\hline Doctor Visits & 0.333 & 0.149 & 0.041 & 0.626 & -0.140 & 0.089 & -0.314 & 0.033 \\
\hline $\mathrm{Rx}$ & 0.027 & 0.020 & -0.012 & 0.066 & -0.052 & 0.018 & -0.088 & -.017 \\
\hline Surgery & 0.001 & 0.004 & -0.008 & 0.009 & -0.001 & 0.007 & -0.014 & 0.012 \\
\hline
\end{tabular}

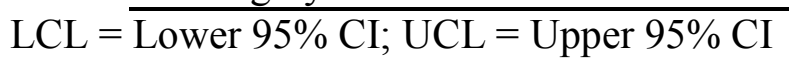




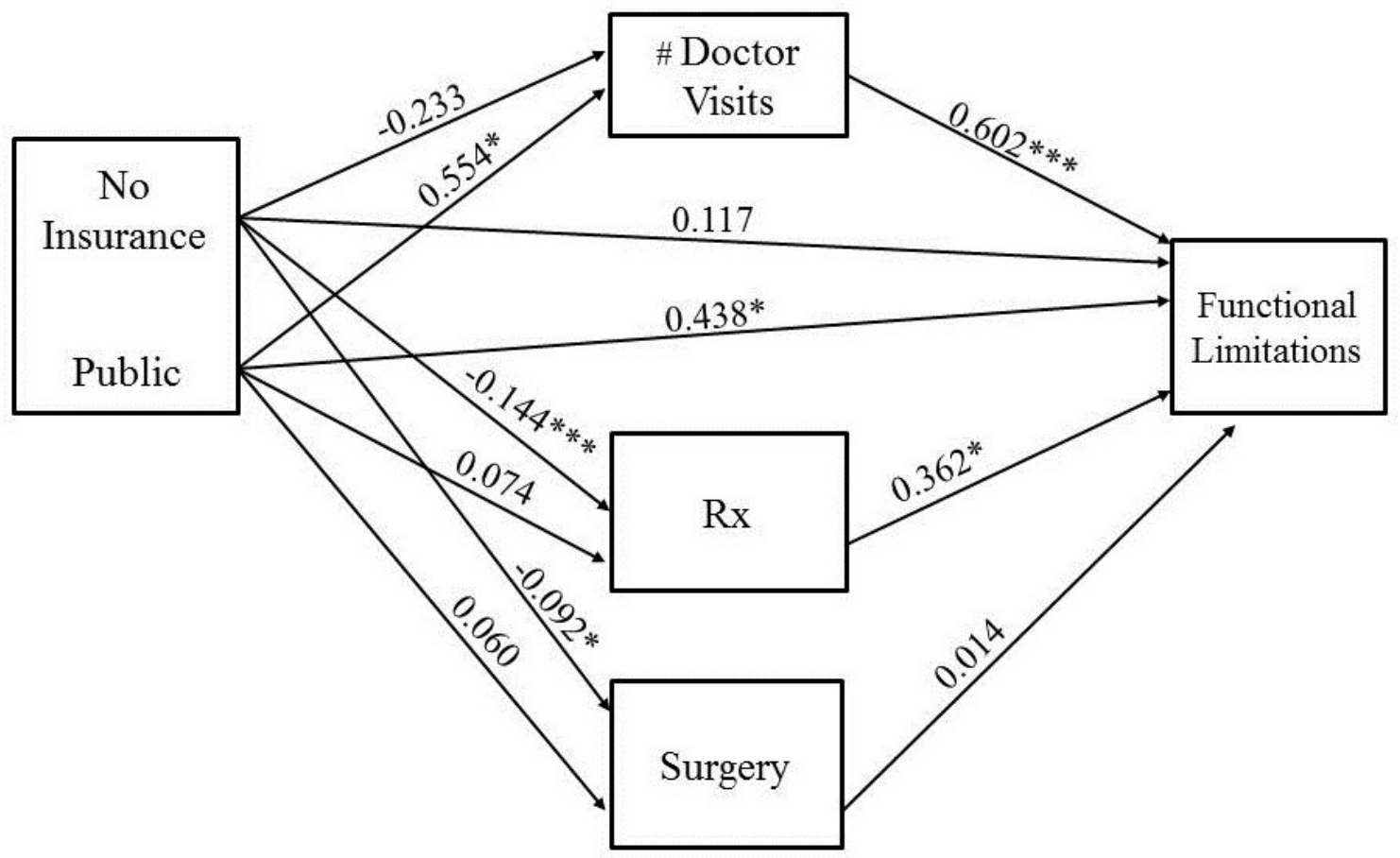

Figure 4-4. Mediation Model Effect Estimates.

$* \mathrm{p}<0.05, * * \mathrm{p}<0.01, * * * \mathrm{p}<0.001$ 
time of injury and then continues to develop more limitations throughout the post-injury period". Trajectory 3 exhibited an increase in functional limitations after injury followed by continual deterioration, satisfying hypothesis 1d. Hypothesis 1e stated "Trajectory analysis will reveal a subgroup that has fluctuating levels of functional limitations over the course of the study". The fifth trajectory identified by the model, Trajectory 4 , differed from the subgroup in hypothesis 1e. Rather than fluctuating in functional status over time, resulting in a flat, intermediate trajectory, Trajectory 4 appears to experience an initial functional deficit after injury but then goes on to consistently improve over time. This subgroup has a similar pattern of change to Trajectory 2, but Trajectory 4 has a significantly higher functional limitations score at baseline. Additionally, members of Trajectory 4 improve during the post-injury period and eventually have a lower functional limitations score than they had at baseline. Therefore hypothesis 1e was rejected.

The second study hypothesis stated "Pre-injury insurance status will be predictive of an individual's recovery subgroup". Regression analyses found that public insurance positively predicted membership in Trajectories 4 and 5, while no insurance was predictive of membership in Trajectory 3. These findings satisfy hypothesis 2 . Hypothesis 2 also had three additional sub-hypotheses. Hypothesis 2a stated "Private insurance will predictive of functional trajectories with less functional impairment". Private insurance was used as the reference category in all regression analyses and findings show that both public insurance and no insurance are predictive of the three trajectories with the highest functional limitation scores (Trajectories 3, 4, and 5). However, public insurance and no insurance were not significant negative predictors of the two trajectories with less functional impairment, indicating that private insurance was not a significant predictor of trajectories with better outcomes. Therefore, hypothesis $2 \mathrm{a}$ is rejected. Hypothesis $2 \mathrm{~b}$ stated "Public insurance will be predictive of functional trajectories with greater functional impairment" and hypothesis $2 \mathrm{c}$ stated "Having no insurance will be predictive of functional trajectories with more functional impairments". Both of these hypotheses were accepted based on the results previously described in this paragraph.

The third study hypothesis stated "Medical care use will mediate the influence of insurance status on long-term functional outcomes after injury." The study found that both public insurance and no insurance had significant indirect effects on functional status, satisfying hypothesis 3. Hypothesis 3a stated that "Outpatient doctor visits will mediate the effect of insurance status on functional outcomes". Doctor visits was a significant mediator of the effect of public insurance but not for the effect of having no insurance. Hypothesis 3a was accepted. Hypothesis $3 b$ stated "Prescription drug use will mediate the effect of insurance status on functional outcomes." Prescription drug use was a significant mediator of the effect of no insurance on functional status, satisfying hypothesis $3 \mathrm{~b}$. Hypothesis $3 \mathrm{c}$ stated "Outpatient surgery will mediate the effect of insurance status on functional outcomes." Surgery was not a significant mediator for the effect of either insurance status. Hypothesis $3 \mathrm{c}$ was rejected. 


\section{CHAPTER 5. DISCUSSION}

\section{Purpose of the Study}

The purpose of this study is to investigate how injury impacts long-term functioning in order to better characterize the recovery process and determine which patients are most at risk for not reaching full recovery. To this end, we identified five subgroups of injured individuals who had distinct trajectories of functioning over time and examined baseline predictors of subgroup membership. A second research objective of this study is to assess whether health insurance and medical care use influence longterm functional status after injury. Specifically, this objective sought to examine whether or not insurance status is a determinant of functional outcomes by way of its effect on medical care use. A mediation analysis was conducted in order to determine if insurance status indirectly affects long-term functional outcomes by influencing the medical care use individuals receive.

\section{Major Findings}

\section{Descriptive Analyses}

The descriptive analyses found that compared to non-injured HRS respondents, the injured study sample was comprised of individuals who were on average significantly younger, more likely to be male, more likely to be high school graduates, and more likely to be living with their spouses. Additionally the injured sample was more likely to be working at baseline and have private insurance. Furthermore, the non-injured respondents had a greater number of health conditions compared to the injured sample. Most baseline differences between the injured and non-injured HRS respondents are likely explained by the fact that a smaller percentage of injured respondents were over 65 at baseline.

When examining characteristics of the injured sample over time, the study found that individuals undergo significant changes in functional status, insurance status, and medical care use throughout the course of the study. On average, there is a decline in functioning during the peri-injury period that is followed by an improvement during the first wave of the post-injury period (wave 3). After wave 3, however, there is a gradual decline in functional status for the rest of the study. Additionally, the average functional status of the injured sample does not return to baseline levels of functioning after injury, suggesting that injury results in lasting functional deficits in this population.

The proportion of individuals with various types of insurance also changes throughout the study. Overall, the percentage of participants with private insurance decreased with time and the number of participants with public insurance increased. Additionally, the number of people with no insurance gradually decreased throughout the study. By the final survey wave, nearly $80 \%$ of participants have public insurance, while only $17.7 \%$ are privately insured and $3 \%$ are uninsured. This is to be expected since the 
majority of respondents were over 56 at baseline. The study spans approximately ten years of time and therefore the majority of respondents should be eligible for Medicare by the final wave of the study.

Lastly, measures of medical care use vary across waves of the study. All medical care use variables show an increase between waves 1 and 2 of the study. This indicates that injury likely results in the use of more medical care. After an increase during the peri-injury period, the mean number of doctor visits slightly declines yet remains higher than baseline for the remainder of the study. The standard deviation for doctor visits is also highest during the peri-injury period, suggesting that the greatest variation in medical care use during the study takes place after injury. The percentage of respondents using prescription drugs also increases after injury. The proportion of individuals using medication continues to increase each wave; however the largest increase occurs between the pre-injury and peri-injury periods. Finally, the percentage of participants undergoing outpatient surgery increases slightly between the pre-injury and peri-injury period and then returns close to pre-injury levels during the first wave of the post-injury period. Overall, the percentage of participants undergoing surgery is relatively constant throughout the 10-year study.

\section{Trajectory Analysis}

The trajectory analysis identified 5 distinct trajectories for the injured study sample. The subgroups are summarized below.

Trajectory 1: Static, High Functioning. This group is characterized as having a consistently low score on the functional limitations scale. $18.9 \%$ of individuals belonged to this subgroup.

Trajectory 2: Dynamic, Big Hit. This group is characterized as having an initial increase in functional limitations after injury followed by gradual recovery over time (i.e. decreasing functional limitations score). However, these individuals do not return to their pre-injury level of functioning and continue to have some functional limitations during the post-injury period. This was the largest subgroup in the study with $46.3 \%$ of the sample belonging to this group.

Trajectory 3: Dynamic, Slow Burn. This group is characterized as having an initial increase in functional limitations after injury followed by a further decline in functional status over time (i.e. increasing functional limitations score). This was the smallest subgroup, with $10.5 \%$ of respondents belonging to it.

Trajectory 4: Dynamic, Long-Term Improvement. This group is characterized as having an increase in functional limitations during the peri-injury period followed by gradual improvement. These individuals' have fewer functional limitations during the post-injury period than the pre-injury period. $13.4 \%$ of study participants belonged to this subgroup. 
Trajectory 5: Static, Low Functioning. This group is characterized as having a consistently high score on the functional limitations scale. $10.8 \%$ of the sample belonged to this subgroup.

Both of the static trajectories (1 and 5) had fairly constant functional limitations scores over the course of the study. Both increased slightly during the peri-injury period, but had relatively flat slopes over time. These two subgroups also differed significantly from one another throughout the study, with Trajectory 5 having a much higher average functional limitations score than Trajectory 1.

The three dynamic trajectories $(2,3$, and 4$)$ also had patterns of functional limitations that were distinct from one another, although most overlapped during at least one time point. Trajectory 2 is most similar to the hypothesized "Big Hit" trajectory, although there is only a small increase in mean functional limitations between the preinjury and peri-injury time points. However, this subgroup's functional limitation score does decrease during the post-injury period, indicating that some functional improvement has possibly taken place. Two of the dynamic trajectories ( 3 and 4 ) had relatively large changes in functional status over the course of the study. Trajectory 3 , in particular, experiences a decline in functioning during wave 2 and continues to acquire more functional limitations in the post-injury period. In contrast, functional limitations increase during wave 2 for Trajectory 4; however, this is followed by steady improvement throughout the remainder of the study.

Baseline descriptive statistics for each subgroup found that the trajectory subgroups differed significantly on all baseline characteristics, with the exception of age and race/ethnicity. Trajectory 1 had the greatest percentage of males, the smallest percentage of older members, and the most members belonging to the highest household assets quintile. Trajectory 1 also had relatively greater percentages of members with bachelor's degrees, members who were currently working, and members with no health conditions. Trajectory 5 had the greatest number of members with public insurance, the greatest percentage of minority members, the greatest percentage of female members, the lowest educational attainment, the smallest percentage of working members, and the smallest percentage of members belonging to coupled households. Trajectory 3 had the highest percentage of members without health insurance.

Regression analyses found several consistent predictors of trajectory subgroups. In general, these analyses showed that women, individuals with multiple health conditions, and individuals with no insurance and public insurance were more likely to belong to trajectories with poorer functional outcomes (Trajectories 3, 4, and 5). In contrast, men, individuals with more assets, individuals with fewer health conditions, and individuals who were working were more likely to belong to trajectories with better functional outcomes (Trajectories 1 and 2). 


\section{Mediation Analysis}

The mediation analysis examined the relationship between insurance status, medical care use, and functional status. This study hypothesized that the effect of insurance status on functional status is mediated by medical care use. In the first step of the analysis, the effect of insurance status on functional status was determined without accounting for medical care use. Regression results showed that public insurance was associated with increased functional limitations relative to private insurance. Not having insurance did not have a significant effect on functional limitations. The second part of the mediation analysis assessed whether insurance status predicts medical care use. The findings indicated that public insurance was associated with increases in the number of doctor visits, but not with medication use or surgery. Being uninsured was significantly associated with a decreased likelihood of using prescription drugs or undergoing surgery, but was not associated with doctor visits. The last model of the mediation analysis examined whether insurance status and medical care use have an effect on functional status in the presence of one another. This analysis found that public insurance, doctor visits, and prescription drug use were positive predictors of functional limitations. Not having insurance and outpatient surgery were not significant predictors of functional limitations in this model.

Overall, the mediation analysis found that the effect of public insurance on functional limitations was partially mediated by medical care use. Although total indirect effect for public insurance was significant, the only significant individual indirect effect was for doctor visits. This indicates that the effect of public insurance on functional status is only mediated by doctor visits. Neither the total effect nor the direct effect of being uninsured on functional status was significant. However, the analysis indicated that there was a significant total indirect effect of being uninsured on functioning. The individual indirect effects indicated that not having insurance reduces medical care use, which in turn increases functional limitations. Prescription drug use was the only significant individual indirect effect. Both doctor visits and surgery were not significant mediators of the effect of not having insurance on functional status.

\section{Comparison of Study Findings to Existing Literature}

\section{Changes in Long-term Functional Status after Injury}

Overall, the findings of this study were consistent with what has been described in the literature. The results of the trajectory analysis were similar to other longitudinal studies that collected information on functional status multiple years after injury. Similar to Sluys et al. (2005), this study found that the majority of injured patients recover from injury; however, they also go on to experience long-term, persistent functional deficits. Additionally, it also found that a subgroup of the injured sample experiences improvements in functioning beyond the first two years of the recovery period. Soberg et al. (2012) found that approximately $20 \%$ of patients reported improved physical health 
between 2 and 5 years post-injury. This is similar to the recovery pattern of individuals belonging to Trajectory 4 . In general, the results reflect what has been reported in the literature in regards to long-term changes in functioning after injury. This study adds to the existing literature by focusing on the all injury population and identifying distinct trajectories of functioning after injury.

\section{Predictors of Long-term Functional Status}

This study found that many of the same predictors of long-term functional status in other studies were significant in this one as well. It has been consistently reported throughout the literature that women are at significantly higher risk for decreased functioning and lower quality of life after injury compared to men (Holbrook et al., 2001; McGeary et al., 2003, Holbrook \& Hoyt, 2004; Polinder, 2007). Gender was a highly significant predictor of functional trajectories in this study, with women being more likely to belong to trajectory subgroups with worse functional outcomes. Pre-injury health status is also commonly reported to be a predictor of post-injury health ouctomes. The literature has shown that poor outcomes are more common in patients with multiple chronic illnesses at the time of injury (McCarthy et al., 1995; Polinder, 2007; Langley, 2011; Reistetter et al., 2011). The results of this study also found that the number of health conditions at baseline was a significant predictor of functional trajectory. Individuals with a greater number of conditions were more likely to belong to trajectories with a greater number of functional limitations. Pre-injury socioeconomic status has also been reported by the literature to affect functional outcomes in injured patients. Higher income and educational attainment are associated significant improvement in functioning within the first year after injury (Mackenzie et al., 1988; Bosse et al., 2002; Holtedahl \& Veiersted, 2007; Soberg et al., 2012). This study had similar results and individuals belonging to quintiles with more assets and individuals with more education were more likely to belong to trajectories with the smallest number of functional limitations. Lastly, the literature identifies social support as a strong predictor of outcome after injury. Social support is associated with greater functional independence and improved quality of life outcomes (Bosse et al., 2002;Farrell et al., 2010; Kiely et al., 2006). This study found that when comparing the two trajectories with the greatest number of functional limitations after injury, individuals in the trajectory that experiences the most improvement are significantly more likely to live with spouses.

Some predictors reported in the literature were not found to be significant in this study. In contrast to Bosse et al. (2002), this study did not identify race to be a significant predictor of functional outcomes. However, the findings did show that the trajectory with the greatest number of functional limitations also had the largest percentage of nonwhite members. Despite this, race/ethnicity was not significant in regression analyses that controlled for other variables. This study also had mixed findings regarding age, possibly because the sample consisted of only individuals over the age of 50. Individuals in Trajectory 5, the subgroup with the most functional limitations, were less likely to belong to older age groups compared to both Trajectory 2 and Trajectory 4 . However, when comparing the two trajectories with the smallest number of functional limitations, 
individuals belonging to Trajectory 1 were less likely to fall into the oldest age category compared to Trajectory 2.

\section{Effect of Insurance Status on Medical Care Use after Injury}

Similar to the previous literature, this study found that insurance status determined medical care use after injury. Public insurance was associated with increases in medical care use, while not having insurance was associated with decreases in medical care use. Publicly insured individuals visited the doctor a greater number of times compared to their privately insured counterparts. Uninsured individuals, however, were less likely to use prescription medication or have outpatient surgery than the privately insured. These results are most similar to those reported by Englum et al. (2011) and Hadley (2007). Englum et al (2011) found that publicly insured patients were more likely to receive post-acute care services compared to privately insured patients. In the injured population, Hadley (2007) found that uninsured patients took significantly fewer prescription medications that those with private insurance.

\section{Effect of Insurance Status and Medical Care Use on Functional Outcomes}

Like the existing literature, the trajectory analysis found that both public insurance and no insurance were significantly associated with worse long-term functional outcomes (Tate et al., 1994; Bosse et al., 2002; Pape et al., 2006; Shafi et al., 2007; Bedell 2008). Public insurance was predictive for Trajectories 4 and 5, which had the greatest number of functional limitations. Having no insurance at baseline was predictive for Trajectory 3, which experiences the greatest deterioration in functioning over time. Furthermore, the mediation analysis found that the direct effect of public insurance, but not being uninsured, was associated with greater functional limitations. The direct effect of no insurance on functional status was not significant, however, the indirect effect was. This indicated that not having insurance increases functional limitations by altering medical care use.

Like other studies, this research also found that the relationship between medical care use and functional outcomes is difficult to assess. Patients who are more severely injured or have more health problems should be expected to use more health services. As a result, some studies report a negative association between increased health service utilization and health outcomes (Guilcher et al., 2010; Davidson et al., 2011). However, other studies have indicated that increased access to healthcare providers is associated with improvements in functional outcome (Kucan et al., 2010). Additionally, not receiving care when it is needed also results in worse outcomes (Castillo et al., 2008). Regression results in this study found that both outpatient doctor visits and medication use were predictive of more functional limitations in this sample. Outpatient surgery was not significantly associated with functional limitations. These findings suggest that access to services, rather than quantity, may be more important in assessing the impact of 
medical care on outcomes. Studying access may reduce the confounding effect of need when studying the relationship between health services and health outcomes.

\section{Research Implications of the Findings}

\section{Patient-Centered Outcomes Research}

The findings of this study have several implications in regards to patient-centered outcomes research on the injured population. First, it demonstrates that significant heterogeneity in the course of recovery after injury exists. Injury is capable of adversely affecting individuals' long-term health by resulting in lasting functional deficits or even changing the course of health altogether. Examining factors associated with changes in functioning over time improves the understanding of causes that alter the progression of functional deficits. Patient-centered outcomes research aims to be able to predict a patient's outcome given their personal characteristics. This study found that each functional trajectory had a unique combination of predictor variables. This information can be used to advance research on assessing patient risk for adverse outcomes and to support decision-making that strives to optimize outcomes for patients within a particular subgroup. Enhanced understanding of the processes that lead to better outcomes within subgroups of the injured population will help clarify which individuals and under what conditions clinical interventions are most effective.

The mediation analysis of this study also provides some information regarding processes of care to target that can be modified to improve outcomes. The findings suggest that insurance status influences the medical care people receive, which goes on to affect their functional outcomes. This study found that patients without insurance are less likely to take prescription medication or have outpatient surgery compared to those with private insurance. Research that identifies the most effective treatments as well as the reasons why individuals do not receive them should be carried out. This information can be used to reduce barriers to care that relate to individuals, healthcare providers, and health insurance.

\section{Health Services Research}

This study highlights several issues in analyzing the effects of medical care use on health outcomes. The results of the mediation analysis illuminate the difficulty in determining the precise effect of medical care use on health outcomes. Like previous studies, this study also found that increased medical care use was associated with increased functional limitations. Health services research that strictly evaluates the efficacy of medical care by examining improvement in outcomes is likely unable to adequately account for the reciprocal nature of the relationship between quantity of care used and severity of health conditions. This is especially true when research is carried out using a heterogeneous population that contains a subgroup of individuals that are heavy 
health services utilizers with severe, chronic health conditions. Health services researchers should be cautious when studying outcomes in populations with significant heterogeneity, such as the injured population.

\section{Public Health Research}

The burden of nonfatal injury is often studied using only severely-injured populations. Although this study did not have information regarding the severity of respondents' injuries, it is likely that most were not life-threatening. The results showed that significant and lasting functional deficits occur in the all-injury population. Additionally, injury is often associated with short-term functional deficits. The findings of this study indicated that post-injury functional limitations persisted for multiple years and, in some individuals, caused continual functional deterioration for the remainder of their lives. The implications of this suggest that more research should be carried out on the all-injury population, as well as on individuals with minor and moderate injuries. This is necessary to better estimate the true burden of nonfatal injury on society.

\section{Clinical Implications of the Findings}

The findings of the trajectory analysis provide insight to practitioners into how older adults' functional outcomes differ after injury. Because less than half of the individuals in this study had a pattern of recovery similar to the implicit mental model of clinicians that Iwashyna (2012) describes, developing specialized care processes for patient subgroups at risk for long-term functional limitations may lead to improved outcomes for a relatively large percentage of injured patients. Patients in Trajectories 3, 4 , and 5 make up roughly one-third of the study sample, yet they do not follow the expected recovery trajectory. They also happen to have the greatest number of functional limitations after injury. Examining each of these trajectories individually and in relation to one another can advance understanding about how functional limitations persist, develop, and improve over time.

\section{Predictors of Functional Decline}

When examining patients in Trajectory 3 , it is obvious that they have functional limitations that not only persist after injury, but also increase with time. There are many possible physiological and psychosocial explanations for the ongoing functional decline in these patients including insufficient rest after injury, an inactivity/loss-of-functioning spiral, self-imposed restriction of activities and mobility, the adoption of a sick role, or exacerbation of other medical conditions (Brown, Roth, Allman, Sawyer, Ritchie, \& Roseman 2009). At baseline, members of Trajectory 3 do not differ significantly from patients in Trajectory 2 in regards to age, work status, or functional limitations; however, they do have significantly more health conditions and are more likely to be uninsured. They also tend to belong to low and medium household assets quintiles, but not 
necessarily the lowest. These findings possibly suggest that patients in Trajectory 3 may be more likely to have underlying health issues that were not properly addressed prior to their injury due to limited financial resources or health insurance coverage. Although they have a similar functional status at baseline compared to Trajectory 2 , they may be in a more fragile health state overall and therefore be less capable of recovering fully after injury. In order to reduce Trajectory 3 patients' downward slope in functioning, clinicians may need to provide more intensive and ongoing care to these patients in order to minimize the number of functional limitations they accrue over time.

Comparing Trajectory 3 patients with Trajectory 4 patients also could provide more information to clinicians about why these individuals experience continual functional decline. Although patients in Trajectory 4 have more functional limitations at baseline than patients in Trajectory 3 , their functional status continues to improve after injury and for the duration of the study. By the end of the study, those in Trajectory 4 end up with fewer functional limitations than those in Trajectory 3. The distribution of individuals with various numbers of health conditions is relatively similar between the two trajectories, with Trajectory 4 having slightly more individuals in the category with the highest number of health conditions. Also, a greater percentage of people in Trajectory 4 are not working and a smaller percentage is uninsured. Additionally, greater percentages of patients in Trajectory 4 are in older age groups and in the higher household assets quintiles. These differences suggest that members of Trajectory 3 may not improve over time like those in Trajectory 4 because more of them are working, uninsured, and have fewer financial resources at the time of their injury. Those in Trajectory 3 may have less time to rest after injury due to their work status and limited financial means. Over time, their functional limitations increase relative to those in Trajectory 4 who potentially have fewer physical demands due to their non-working status. Increased financial resources, insurance coverage, and more time off may help explain why those in Trajectory 4 have long-term improvement while those in Trajectory 3 have continuous decline. This information may suggest that clinicians could improve outcomes by encouraging working patients to discuss their injuries with employers. Employers could potentially accommodate employees during their recovery by reducing the physical demands placed on them. Employers could also remove barriers that inhibit employees from receiving follow-up care by offering paid time off for doctor visits.

\section{Predictors of Functional Improvement}

Because Trajectory 4 experiences the greatest amount of improvement after injury, examining its characteristics may provide explanations for how patients re-gain functional abilities after losing them. Both Trajectory 4 and 5 have more functional limitations at baseline than any of the other trajectory subgroups. While Trajectory 4 goes down a path of long-term improvement after injury and fully recovers, Trajectory 5 is characterized by a persistent, high number of functional limitations. However, it is important to note that Trajectory 5 does have a slight decrease in mean functional limitations after injury, indicating that a reversal of the loss of functioning is possible in at least some patients in this trajectory. Aside from age, the only characteristic that 
significantly predicts membership in Trajectory 4 relative to Trajectory 5 is living in a coupled household. Individuals in Trajectory 4 are $68 \%$ more likely to live with a spouse than individuals in Trajectory 5. Living with a spouse may benefit injured patients by providing social support, sharing the financial burden of medical care and lost wages, assisting patients with caring for their injuries at home, providing transportation to medical appointments, and maintaining private health insurance should the injured person lose their insurance coverage by not being able to work. In light of this, clinicians may improve outcomes of patients living alone by ensuring that patients are capable of caring for their injuries themselves, and, if not, recommending home health care services. Additionally, case managers may want to increase the frequency of contact during the follow-up care period and ensure that patients have made transportation arrangements in advance of their follow-up appointments.

\section{Clinical Benchmarks after Injury}

Unique clinical benchmarks for assessing recovery after injury should be adopted in order to evaluate the quality of recovery for patients with distinct trajectories. Benchmarks should specifically take into account the longitudinal nature of recovery, particularly in older adults who require more time to heal. Measurements of functional limitations should be taken at multiple time points after injury so that both the rate and magnitude of recovery or decline can be evaluated. This is especially important early in the recovery phase, where trajectories may be more easily redirected by altering some aspect of patient care or behavior. Additionally, asking patients to report variations in functioning between measurements can also help clinicians determine how stationary a patient's recovery is. For example, some patients may experience great variability in their day-to-day functioning which would change how clinicians perceive a positive measurement following a negative measurement, or vice versa. For patients prone to acute exacerbations, performing a thorough assessment of triggers that impair functioning could provide patients with information that helps them have more days free of impairment.

\section{Policy Implications of the Findings}

\section{Policies Concerning the Coverage of Medical Services}

As mentioned under the section on "Research Implications of the Findings", this study elucidates the difficulty in determining the precise effect of medical care use on health outcomes for a heterogeneous population. As a result, the decision of whether or not to cover a particular procedure or medication may not be based on accurate evidence. Both public and private insurers should take into account personal characteristics of their beneficiaries when determining which services to cover. 
Additionally, coverage decisions should take into account that beneficiaries' outcomes are actually health trajectories. Insurers will likely be paying for services beyond the acute phase of injury and should therefore endeavor to provide coverage for services that mitigate the likelihood of an individual experiencing an adverse health trajectory. This is especially true for government programs such as Medicare that will be paying for services for the rest of a person's life. Increasing the intensity and duration of care in the short-term may produce better long-term outcomes.

\section{Policies Expanding Access to Medical Care after Injury for Older Adults under 65}

This study found that older adults with no insurance were more likely to have poor long-term functional outcomes. Specifically, not having insurance was predictive of a functional trajectory that exhibited an initial increase in functional limitations after injury followed by a further decline in functional status over time. The mediation analysis also found that being uninsured significantly reduced the likelihood of using prescription medication and undergoing outpatient surgery. It is possible that older adults without insurance do not receive adequate care for their injuries which sets them on a course of functional deterioration. Ensuring that these adults receive sufficient care immediately after their injury as well as follow-up care may help them enter a trajectory of recovery rather than dysfunction.

Because most of the individuals in this study ultimately enroll in Medicare, the public cost of individuals following a trajectory of declining functional ability is evident. In order to improve outcomes and reduce long-term costs to public programs, policies should be put in place that increase access to medical care that injured, older adults could not otherwise afford. Such policies could potentially save the public money by improving the health of future beneficiaries.

\section{Study Limitations}

\section{Lack of Direct Information on Respondent Injury Severity}

The primary limitation of this study is lack of information pertaining to the study participant's injury. Because the survey does not ask participants to describe in detail the nature, severity, and treatment received for their injury, it is difficult to account for the

role injury characteristics play in determining long-term outcomes. Although clinical data on injury severity would strengthen this study, there has been considerable debate on how well routine, hospital-collected measures provide indicators of severity. Two common clinical severity indicators, admission to a hospital and length of stay, have been shown to be unreliable predictors of long-term outcomes in the injury population (Barker, Power, \& Roberts, 1996; Cryer, 2005; Cryer \& Langley, 2006; Langley et al., 2007; Ly, Travison, Castillo, Bosse, \& MacKenzie, 2008). For example, one study found that 50$75 \%$ of pediatric injuries that resulted in permanent disability were treated in the 
outpatient setting (Barker et al., 1996). Other issues with utilizing hospital discharge data for long-term outcomes involve deficiencies and discrepancies in hospital reporting. Studies have found that hospitals frequently utilize "unspecified" codes for injured patients and that there is significant variability and inconsistency in regards to injury coding practices (Langley et al., 2007). Large amounts of missing and miscoded information relating to injury could be problematic for a small cohort study, such as this one. Additionally, studies have shown that injuries receiving no medical treatment can cause significant long-term functional limitations. Relatively little is reported on outcomes after injuries that do not result in hospitalization and many have called for studies on a more comprehensive, "all injury" population (Polinder et al., 2010).

By using self-report to identify injury cases, people who experienced a significant injury that were not treated at a hospital were included in the study. Although there is no objective data available on injury severity, allowing respondents to be included that selfidentify as injured may make this study more encompassing of the all injury population.

\section{Validity of Self-reported Data}

Another limitation of the proposed study is that data on injury, as well as all variables, is generated from self-report. Assignment to the study group is based on an affirmative answer to survey questions that inquire about recent accidents and injuries. An individual's ability to recall and report only significant injuries over a two-year period may result in either under- or over-reporting of injury occurrences. For the former, Zwerling et al (1995) have shown that self-reported surveys with longer recall periods, specifically the HRS, can be used to estimate injury rates. Their study determined that even in population subgroups most likely to be affected by recall bias such as those with lower incomes, less education, depression, and poor short-term memory, there was $10 \%$ or less bias in reporting when compared to those who were less likely to have problems with injury recall (Zwerling et al., 1995). In regards to the limitation surrounding significance of the self-reported injury, it is possible that some study participants will be included in the injury group who would have not been included had injured participants been identified by hospital discharge or claims data. Studies have found that individuals are more likely to under-report injury and injuries that are less severe or do not result in missed work often go unreported (Zwerling et al., 1995; Landen \& Hendricks, 1995). This indicates that self-report is still a relatively conservative method for identifying injured individuals.

\section{Observation Interval Length}

The HRS survey is conducted every two years and this may make it difficult to capture brief, yet large decreases in functional ability between interview waves. If shorter intervals between measurements were available, information regarding the magnitude of recovery could be more easily determined. However, this study is primarily concerned with long-term outcomes and lasting functional deficits. Although knowledge of short- 
term functional deficits is useful, this study is investigating measures of functional status that represent an individual's functional ability overall for the specified time interval.

Additionally, the static reporting of HRS measurements could also cause participants with only short-term functional deficits to be characterized as having a lower functional status at that point in time than they have on average. Because this study evaluates functional trajectories using outcomes that are measured at six time points over a ten-year period, the problems associated with static reporting are somewhat reduced.

\section{Recommendations for Future Research}

This study examined long-term functional limitations after injury in the older adult population; however, injury affects individuals over the entire course of the life span. Future studies should incorporate all ages of the population in order to determine differences in recovery patterns for different age groups. Such studies would help to validate trajectories of recovery after injury. Additionally, studying a wider range of ages would make comparisons between individuals with different insurance types more relevant. The proportion of uninsured individuals in the study sample was relatively small due to the age groups studied. Including younger individuals in the study sample would make it easier to detect the effect of insurance status on outcomes.

Additionally, future research on the all-injury population would be strengthened by having more specific information on the nature of individual injuries. This would allow for the comparison of recovery patterns for different types of injury. It would also help to identify what types of injuries put people most at risk for long-term functional deficits. Various nuances such as the mechanism and place of injury could also be explored.

Future research should also attempt to collect more detailed information on health insurance coverage and medical care use for injured individuals. This study evaluated the effect of health insurance and medical care use on functional outcomes using relatively broad constructs. Additional information, particularly regarding rehabilitative service utilization, would have strengthened this study. More precise information on what medical care was recommended to patients and what was actually obtained would add to future studies. This information would advance injury research by examining how treatment recommendations differ across patients and why patients do not receive all recommended care.

\section{Conclusion}

To the researcher's knowledge, this is the first study investigating long-term functional outcomes of the all-injury population in the United States. These results add to the literature by providing a description of distinct courses of recovery after injury in the

older adult population. Furthermore, several significant predictors of functional 
trajectories were identified. This study also demonstrated that insurance status is a significant predictor of both functional outcomes and medical care use after injury. Insurance status was found to exert its effect on health outcomes both directly and indirectly through medical care.

Ultimately, the findings from this study are beneficial to several fields of study including public health, health outcomes, and health policy. Research that aims to improve the understanding of how individuals' health outcomes differ and the causal processes that determine these outcomes will lead to policies and treatments that improve the quality of life for injured individuals. 


\section{LIST OF REFERENCES}

Adams P, Kirzinger W, and Martinez M. (2013). Summary health statistics for U.S. adults: National Health Interview Survey, 2012. National Center for Health Statistics. Vital Health Stat 10(259). 2013.

Aitken LM, Chaboyer W, Kendall E, Burmeister E. (2012). Health status after traumatic injury. J Trauma Acute Care Surg. 72(6):1702-8.

Alban RF, Berry C, Ley E, Mirocha J, Margulies DR, Tillou A, Salim A. (2010). Does health care insurance affect outcomes after traumatic brain injury? Analysis of the National Trauma Databank. Am Surg 76(10):1108-11.

Andelic N, Hammergren N, Bautz-Holter E, Sveen U, Brunborg C, Røe C. (2009). Functional outcome and health-related quality of life 10 years after moderate-tosevere traumatic brain injury. Acta Neurol Scand 120(1):16-23.

Andelic N, Bautz-Holter E, Ronning P, Olafsen K, Sigurdardottir S, Schanke AK, Sveen U, Tornas S, Sandhaug M, Roe C. (2012). Does an early onset and continuous chain of rehabilitation improve the long-term functional outcome of patients with severe traumatic brain injury? J Neurotrauma 1;29(1):66-74.

Arango-Lasprilla JC, Rosenthal M, Deluca J, Komaroff E, Sherer M, Cifu D, Hanks R. (2007). Traumatic brain injury and functional outcomes: does minority status matter? Brain Inj. 21(7):701-8.

Arango-Lasprilla JC, Ketchum JM, Francis K, Lewis A, Premuda P, Wehman P, Kreutzer J. (2010). Race, ethnicity, and employment outcomes 1,5 , and 10 years after spinal cord injury: a longitudinal analysis. $P M R$. 2(10):901-10.

Arango-Lasprilla JC, Ketchum JM, Lewis AN, Krch D, Gary KW, Dodd BA Jr. Racial and ethnic disparities in employment outcomes for persons with traumatic brain injury: a longitudinal investigation 1-5 years after injury. (2011). PMR. 3(12):1083-91.

Archdeacon MT, Simon PM, Wyrick JD. (2007). The influence of insurance status on the transfer of femoral fracture patients to a level-I trauma center. J Bone Joint Surg Am 89(12):2625-31

Arrandale V, Koehoorn M, MacNab Y, and Kennedy SM. (2006). How to use SAS巴 Proc Traj and SAS ${ }^{\circledR}$ Proc Glimmix in respiratory epidemiology. School of Occupational \& Environmental Hygiene, Department of Health Care \& Epidemiology, University of British Columbia; Vancouver

Barker M, Power C, Roberts I. (1996). Injuries and the risk of disability in teenagers and young adults. Arch Dis Child. 75:156-158.

Barkley RA, Murphy KR. (2010). Impairment in occupational functioning and adult ADHD: the predictive utility of executive function (EF) ratings versus EF tests. Arch Clin Neuropsychol 25(3):157-73

Baron, R. M. and Kenny, D. A. (1986). The moderator-mediator variable distinction in social psychological research: Conceptual, strategic, and statistical considerations. Journal of Personality and Social Psychology, 51: 1173-1182.

Bedell GM. (2008). Functional outcomes of school-age children with acquired brain injuries at discharge from inpatient rehabilitation. Brain Inj 22(4):313-24. 
Bentler SE, Liu L, Obrizan M, Cook EA, Wright KB, Geweke JF, Chrischilles EA, Pavlik CE, Wallace RB, Ohsfeldt RL, Jones MP, Rosenthal GE, Wolinsky FD. (2009). The aftermath of hip fracture: discharge placement, functional status change, and mortality. Am J Epidemiol 15;170(10):1290-9.

Berdahl TA, Zodet M. (2010). Medical care utilization for work-related injuries in the United States 2002-2006. Med Care 48(7):645-51.

Bosse MJ, MacKenzie EJ, Kellam JF, Burgess AR, Webb LX, Swiontkowski MF, Sanders RW, Jones AL, McAndrew MP, Patterson BM, McCarthy ML, Travison TG, Castillo RC. (2002). An analysis of outcomes of reconstruction or amputation after leg-threatening injuries. $N$ Engl J Med 12;347(24):1924-31.

Bookman, A., Harrington, M., Pass, L., \& Reisner, E. (2007). Family caregiver handbook: Finding elder care resources in Massachusetts. Cambridge, MA: Massachusetts Institute of Technology.

Brackett MA, Rivers SE, Shiffman S, Lerner N, Salovey P. (2006). Relating emotional abilities to social functioning: a comparison of self-report and performance measures of emotional intelligence. J Pers Soc Psychol. 91(4):780-95.

Brede E1, Mayer TG, Gatchel RJ. (2012). Prediction of failure to retain work 1 year after interdisciplinary functional restoration in occupational injuries. Arch Phys Med Rehabil. 93(2):268-74

Brissos S, Balanzá-Martinez V, Dias VV, Carita AI, Figueira ML. (2011). Is personal and social functioning associated with subjective quality of life in schizophrenia patients living in the community? Eur Arch Psychiatry Clin Neurosci 261(7):50917.

Brown RL. (2010). Epidemiology of injury and the impact of health disparities. Curr Opin Pediatr 22(3):321-5.

Brown, C. J., Roth, D. L., Allman, R. M., Sawyer, P., Ritchie, C. S., \& Roseman, J. M. (2009). Trajectories of life-space mobility after hospitalization. Annals of Internal Medicine, 150(6), 372-378.

Butcher JL, MacKenzie EJ, Cushing B, Jurkovich G, Morris J, Burgess A, McAndrew M, Swiontkowski M. (1996). Long-term outcomes after lower extremity trauma. $J$ Trauma 41(1):4-9.

Castillo RC, MacKenzie EJ, Webb LX, Bosse MJ, Avery J; LEAP Study Group. (2005). Use and perceived need of physical therapy following severe lower-extremity trauma. Arch Phys Med Rehabil 86(9):1722-8.

Castillo RC, MacKenzie EJ, Wegener ST, Bosse MJ; LEAP Study Group. (2006). Prevalence of chronic pain seven years following limb threatening lower extremity trauma. Pain 124(3):321-9.

Castillo RC, MacKenzie EJ, Archer KR, Bosse MJ, Webb LX; LEAP Study Group. (2008). Evidence of beneficial effect of physical therapy after lower-extremity trauma. Arch Phys Med Rehabil 89(10):1873-9.

Castillo RC, Mackenzie EJ, Bosse MJ; LEAP Study Group. (2011). Orthopaedic trauma clinical research: is 2-year follow-up necessary? Results from a longitudinal study of severe lower extremity trauma. J Trauma 71(6):1726-31. 
Castillo RC, Wegener ST, Heins SE, Haythornthwaite JA, Mackenzie EJ, Bosse MJ; LEAP Study Group. (2013). Longitudinal relationships between anxiety, depression, and pain: results from a two-year cohort study of lower extremity trauma patients. Pain 154(12):2860-6

CDC. (2012). Web-based Injury Statistics Query and Reporting System (WISQARS). U.S. Department of Health and Human Services, CDC, National Center for Injury Prevention and Control. Available at http://www.cdc.gov/injury/wisqars/index.html

Chan L, Doctor J, Temkin N, MacLehose RF, Esselman P, Bell K, Dikmen S. (2001). Discharge disposition from acute care after traumatic brain injury: the effect of insurance type. Arch Phys Med Rehabil 82(9):1151-4.

Claridge JA, Croce MA, Weinberg JA, Forsythe RM, Miller C, Fabian TC. (2006). The real predictors of disposition in patients with spinal cord injuries. J Trauma 60(1):178-86.

Cleary PD, Fowler FJ Jr, Weissman J, Massagli MP, Wilson I, Seage GR 3rd, Gatsonis C, Epstein A. (1993). Health-related quality of life in persons with acquired immune deficiency syndrome. Med Care 31(7):569-80.

Cobaugh DJ, Angner E, Kiefe CI, Ray MN, Lacivita CL, Weissman NW, Saag KG, Allison JJ. (2008). Effect of racial differences on ability to afford prescription medications. Am J Health Syst Pharm. 15;65(22):2137-43

Coleman EA, Kramer AM, Kowalsky JC, Eckhoff D, Lin M, Hester EJ, Morgenstern N, Steiner JF. (2000). A comparison of functional outcomes after hip fracture in group/staff HMOs and fee-for-service systems. Eff Clin Pract 3(5):229-39.

Collie A, Prang KH. (2013). Patterns of healthcare service utilisation following severe traumatic brain injury: an idiographic analysis of injury compensation claims data. Injury 44(11):1514-20.

Corso P, Finkelstein E, Miller T, Fiebelkorn I, Zaloshnja E. (2006). Incidence and lifetime costs of injuries in the United States. Injury Prevention. 12(4):212-218.

Cryer C. (2005). Injury outcome indicators--validation matters. Int J Inj Contr Saf Promot. 12:219-24; discussion 225.

Cryer C, Langley JD. (2006). Developing valid indicators of injury incidence for "all injury". Inj Prev. 12:202-207. doi: 10.1136/ip.2006.011635.

Davidson GH, Hamlat CA, Rivara FP, Koepsell TD, Jurkovich GJ, Arbabi S. (2011). Long-term survival of adult trauma patients. JAMA 9;305(10):1001-7.

Derrett S, Davie G, Ameratunga S, Wyeth E, Colhoun S, Wilson S, Samaranayaka A, Lilley R, Hokowhitu B, Hansen P, Langley J. (2011). Prospective Outcomes of Injury Study: recruitment, and participant characteristics, health and disability status. Inj Prev 17(6):415-8.

Derrett S, Samaranayaka A, Wilson S, Langley J, Ameratunga S, Cameron ID, Lilley R, Wyeth E, Davie G. (2012). Prevalence and predictors of sub-acute phase disability after injury among hospitalised and non-hospitalised groups: a longitudinal cohort study. PLoS One 7(9):e44909.

Derrett S, Wilson S, Samaranayaka A, Langley J, Wyeth E, Ameratunga S, Lilley R, Davie G, Mauiliu M. (2013). Prevalence and predictors of disability 24-months after injury for hospitalised and non-hospitalised participants: results from a longitudinal cohort study in new zealand. PLoS One 8(11):e80194. 
Donaldson SI. (2001). Mediator and moderator analysis in program development. In S. Sussman (Ed.), Handbook of Program Development for Health Behavior Research and Practice (pp 470-500). Thousand Oaks, CA: Sage Publications, Inc.

Doyle JJ Jr. (2005). Health insurance, treatment and outcomes: using auto accidents as health shocks. Rev Econ Stat. 87(2):256-270

Du Bois M, Donceel P. (2010). Outcome and cost of spinal fractures and spinal tumors. Eur Spine J 19 Suppl 1:S74-8.

Englum BR, Villegas C, Bolorunduro O, Haut ER, Cornwell EE 3rd, Efron DT, Haider AH. (2011). Racial, ethnic, and insurance status disparities in use of posthospitalization care after trauma. J Am Coll Surg 213(6):699-708.

Erbes CR, Kaler ME, Schult T, Polusny MA, Arbisi PA. (2011). Mental health diagnosis and occupational functioning in National Guard/Reserve veterans returning from Iraq. J Rehabil Res Dev 48(10):1159-70.

Erosa NA, Berry JW, Elliott TR, Underhill AT, Fine PR. (2013). Predicting quality of life 5 years after medical discharge for traumatic spinal cord injury. Br J Health Psychol 9.

Evans S, Airey M, Chell S, Connelly J, Rigby A, and Tennant A. (2003). Disability in young adults following major trauma: 5 year follow up of survivors. BMC Public Health, 3(1):8

Farrell RT, Bennett BK, Gamelli RL. (2010). An analysis of social support and insurance on discharge disposition and functional outcomes in patients with acute burns. $J$ Burn Care Res 31(3):385-92

Gary KW, Arango-Lasprilla JC, Stevens LF. (2009). Do racial/ethnic differences exist in post-injury outcomes after TBI? A comprehensive review of the literature. Brain Inj. 23(10):775-89.

Gorey KM, Luginaah IN, Holowaty EJ, Zou G, Hamm C, Bartfay E, Kanjeekal SM, Balagurusamy MK, Haji-Jama S, Wright FC. (2012). Effects of being uninsured or underinsured and living in extremely poor neighborhoods on colon cancer care and survival in California: historical cohort analysis, 1996-2011. BMC Public Health 24;12:897

Guilcher SJ, Munce SE, Couris CM, Fung K, Craven BC, Verrier M, Jaglal SB. (2010). Health care utilization in non-traumatic and traumatic spinal cord injury: a population-based study. Spinal Cord 48(1):45-50.

Haas JS, Goldman L. (1994). Acutely injured patients with trauma in Massachusetts: differences in care and mortality, by insurance status. Am J Public Health 84(10):1605-8.

Hadley J. (2003). Sicker and poorer--the consequences of being uninsured: a review of the research on the relationship between health insurance, medical care use, health, work, and income. Med Care Res Rev 60(2 Suppl):3S-75S.

Hadley J. (2007). Insurance coverage, medical care use, and short-term health changes following an unintentional injury or the onset of a chronic condition. JAMA 14;297(10):1073-84.

Hakmeh W, Barker J, Szpunar SM, Fox JM, Irvin CB. (2010). Effect of race and insurance on outcome of pediatric trauma. Acad Emerg Med 17(8):809-12

Harris IA, Young JM, Rae H, Jalaludin BB, Solomon MJ. (2006). Predictors of general health after major trauma. J Trauma. 64(4):969-74. 
Hayes AF and Preacher KJ. (2013). Statistical mediation analysis with a multicategorical independent variable. Br J Math Stat Psychol. (ahead of print)

Health Cost Utilization Project. (2007). "Statistics on Hospital-Based Care in the United States, 2007”. Agency for Healthcare Research and Quality. Available from: http://www.hcupus.ahrq.gov/reports/factsandfigures/2007/pdfs/FF_report_2007.pdf.

Heffernan DS, Vera RM, Monaghan SF, Thakkar RK, Kozloff MS, Connolly MD, Gregg SC, Machan JT, Harrington DT, Adams CA Jr, Cioffi WG. (2011). Impact of socioethnic factors on outcomes following traumatic brain injury. J Trauma 70(3):527-34.

Henly SJ, Wyman JF, Findorff MJ. (2011). Health and illness over time: the trajectory perspective in nursing science. Nurs Res 60(3 Suppl):S5-14

Hindmarsh D, Hayen A, Finch C, and Close J. (2009). Relative survival after hospitalization for hip fracture in older people in New South Wales, Australia. Osteoporosis International 20:221-229

Holbrook TL, Anderson JP, Sieber WJ, Browner D, Hoyt DB. (1998). Outcome after major trauma: discharge and 6-month follow-up results from the Trauma Recovery Project. J Trauma 45(2):315-23

Holbrook TL, Anderson JP, Sieber WJ, Browner D, Hoyt DB. (1999). Outcome after major trauma: 12-month and 18-month follow-up results from the Trauma Recovery Project. J Trauma 46(5):765-71

Holbrook TL, Hoyt DB, Stein MB, Sieber WJ. (2001). Perceived threat to life predicts posttraumatic stress disorder after major trauma: risk factors and functional outcome. J Trauma 51(2):287-92

Holbrook TL, Hoyt DB, Stein MB, Sieber WJ. (2002). Gender differences in long-term posttraumatic stress disorder outcomes after major trauma: women are at higher risk of adverse outcomes than men. J Trauma 53(5):882-8.

Holbrook TL, Hoyt DB. (2004). The impact of major trauma: quality-of-life outcomes are worse in women than in men, independent of mechanism and injury severity. $J$ Trauma 56(2):284-90.

Holtedahl R, Veiersted KB. (2007). Physical, social and emotional function after work accidents: a medicolegal perspective. Work 28(4):363-70.

Hu X, Wesson DE, Logsetty S, Spence LJ. (1994). Functional limitations and recovery in children with severe trauma: a one-year follow-up. J Trauma 37(2):209-13.

Inaba K, Goecke M, Sharkey P, Brenneman F. (2003). Long-term outcomes after injury in the elderly. $J$ Trauma 54(3):486-91.

Iwashyna TJ. (2012). Trajectories of recovery and dysfunction after acute illness, with implications for clinical trial design. Am J Respir Crit Care Med 15;186(4):302-4.

Jacoby SF, Ackerson TH, Richmond TS. (2006). Outcome from serious injury in older adults. J Nurs Scholarsh 38(2):133-40.

Jones BL and Nagin DS. (2007). Advances in Group-Based Trajectory Modeling and an SAS Procedure for Estimating Them. Sociological Methods Research 35(4): 542571

Kane RL, Chen Q, Blewett LA, Sangl J. (1996). Do rehabilitative nursing homes improve the outcomes of care? J Am Geriatr Soc 44(5):545-54. 
Kane RL, Chen Q, Finch M, Blewett L, Burns R, Moskowitz M. (1998). Functional outcomes of posthospital care for stroke and hip fracture patients under medicare. $J$ Am Geriatr Soc 46(12):1525-33..

Kane RL, Chen Q, Finch M, Blewett L, Burns R, Moskowitz M. (2000). The optimal outcomes of post-hospital care under medicare. Health Serv Res 35(3):615-61.

Keogh JP, Nuwayhid I, Gordon JL, Gucer PW. (2000). The impact of occupational injury on injured worker and family: outcomes of upper extremity cumulative trauma disorders in Maryland workers. Am J Ind Med 38(5):498-506.

Kendrick D, O'Brien C, Christie N, Coupland C, Quinn C, Avis M, Barker M, Barnes J, Coffey F, Joseph S, Morris A, Morriss R, Rowley E, Sleney J, Towner E. (2011). The impact of injuries study. multicentre study assessing physical, psychological, social and occupational functioning post injury--a protocol. BMC Public Health 31;11:963.

Kendrick D1, Vinogradova Y, Coupland C, Christie N, Lyons RA, Towner EL. (2012). Getting back to work after injury: the UK Burden of Injury multicentre longitudinal study. BMC Public Health. 12:584.

Keyes KB, Wickizer TM, Franklin G. (2001). Two-year health and employment outcomes among injured workers enrolled in the Washington State Managed Care Pilot Project. Am J Ind Med 40(6):619-26.

Kiely JM, Brasel KJ, Weidner KL, Guse CE, Weigelt JA. (2006). Predicting quality of life six months after traumatic injury. J Trauma 61(4):791-8.

Koehoorn M, Breslin FC, Xu F. (2008). Investigating the longer-term health consequences of work-related injuries among youth. J Adolesc Health. 43(5):46673.

Koehoorn M, Xu F, Village J, Trask C, Teschke K. (2010). Back injury trajectories in heavy industries: defining outcomes for epidemiological research. J Occup Environ Med 52(9):908-12.

Krause JS, Saladin LK, Adkins RH. (2009). Disparities in subjective well-being, participation, and health after spinal cord injury: a 6-year longitudinal study. Neuro Rehabilitation. 24(1):47-56.

Kucan J, Bryant E, Dimick A, Sundance P, Cope N, Richards R, Anderson C. (2010). Systematic care management: a comprehensive approach to catastrophic injury management applied to a catastrophic burn injury population--clinical, utilization, economic, and outcome data in support of the model. J Burn Care Res 31(5):692700.

Landen DD, Hendricks S. (1995). Effect of recall on reporting of at-work injuries. Public Health Rep. 110:350-354.

Langley JD, Davie GS, Simpson JC. (2007). Quality of hospital discharge data for injury prevention. Inj Prev 13:42-44

Langley J, Derrett S, Davie G, Ameratunga S, Wyeth E. (2011). A cohort study of shortterm functional outcomes following injury: the role of pre-injury sociodemographic and health characteristics, injury and injury-related healthcare. Health Qual Life Outcomes. 18;9:68. 
Langley J, Davie G, Wilson S, Lilley R, Ameratunga S, Wyeth E, Derrett S. (2013). Difficulties in functioning 1 year after injury: the role of preinjury sociodemographic and health characteristics, health care and injury-related factors. Arch Phys Med Rehabiln 94(7):1277-86

Leidy NK. (1994). Functional status and the forward progress of merry-go-rounds: toward a coherent analytical framework. Nurs Res 43(4):196-202.

Leukhardt WH, Golob JF, McCoy AM, Fadlalla AM, Malangoni MA, Claridge JA. (2010). Follow-up disparities after trauma: a real problem for outcomes research. Am J Surg. 199(3):348-52

Lilley R, Davie G, Ameratunga S, Derrett S. (2012). Factors predicting work status 3 months after injury: results from the Prospective Outcomes of Injury Study. BMJ Open. 2012 Mar 2;2(2)

Lim HJ, Hoffmann R, Brasel K. (2007). Factors influencing discharge location after hospitalization resulting from a traumatic fall among older persons. J Trauma 63(4):902-7.

Livingston DH, Tripp T, Biggs C, Lavery RF. (2009). A fate worse than death? Longterm outcome of trauma patients admitted to the surgical intensive care unit. $J$ Trauma 67(2):341-8; discussion 348-9.

Ly TV, Travison TG, Castillo RC, Bosse MJ, MacKenzie EJ; LEAP Study Group. (2008). Ability of lower-extremity injury severity scores to predict functional outcome after limb salvage. J Bone Joint Surg Am 90(8):1738-43.

MacKenzie EJ, Siegel JH, Shapiro S, Moody M, Smith RT. (1988). Functional recovery and medical costs of trauma: an analysis by type and severity of injury. J Trauma 28(3):281-97.

MacKenzie EJ, Morris JA Jr, Jurkovich GJ, Yasui Y, Cushing BM, Burgess AR, DeLateur BJ, McAndrew MP, Swiontkowski MF. (1998). Return to work following injury: the role of economic, social, and job-related factors. $A m \mathrm{~J}$ Public Health 88(11):1630-7.

MacKenzie EJ, Bosse MJ, Pollak AN, Webb LX, Swiontkowski MF, Kellam JF, Smith DG, Sanders RW, Jones AL, Starr AJ, McAndrew MP, Patterson BM, Burgess AR, Castillo RC. (2005). Long-term persistence of disability following severe lower-limb trauma. Results of a seven-year follow-up. J Bone Joint Surg Am 87(8):1801-9.

MacKenzie EJ, Bosse MJ, Kellam JF, Pollak AN, Webb LX, Swiontkowski MF, Smith DG, Sanders RW, Jones AL, Starr AJ, McAndrew MP, Patterson BM, Burgess AR, Travison T, Castillo RC. (2006). Early predictors of long-term work disability after major limb trauma. J Trauma 61(3):688-94.

MacKenzie EJ, Bosse MJ. (2006). Factors influencing outcome following limbthreatening lower limb trauma: lessons learned from the Lower Extremity Assessment Project (LEAP). J Am Acad Orthop Surg 14(10 Spec No.):S205-10.

MacKinnon, D. P., \& Dwyer, J. H. (1993). Estimating mediated effects in prevention studies. Evaluation Review, 17:144-158.

Malt UF, Blikra G, Høivik B. (1989). The three-year biopsychosocial outcome of 551 hospitalized accidentally injured adults. Acta Psychiatr Scand Suppl. 355:84-93. 
Mayo NE, Nadeau L, Levesque L, Miller S, Poissant L, Tamblyn R. (2005). Does the addition of functional status indicators to case-mix adjustment indices improve prediction of hospitalization, institutionalization, and death in the elderly? Med Care 43(12):1194-202.

Mayou R and Bryant B. (2002). Outcome 3 years after a road traffic accident. Psychological medicine, 32(4):671-675

McCarthy ML, MacKenzie EJ, Bosse MJ, Copeland CE, Hash CS, Burgess AR. (1995). Functional status following orthopedic trauma in young women. J Trauma 39(5):828-36; discussion 836-7.

McCarthy ML, Ewashko T, MacKenzie EJ. (1998). Determinants of use of outpatient rehabilitation services following upper extremity injury. J Hand Ther 11(1):32-8.

McCarthy ML, Dikmen SS, Langlois JA, Selassie AW, Gu JK, Horner MD. (2006). Selfreported psychosocial health among adults with traumatic brain injury. Arch Phys Med Rehabil. 87(7):953-61.

McClellan M. (1998). "Health Events, Health Insurance and Labor Supply: Evidence from the Health and Retirement Survey," in D. Wise, ed., Frontiers in the Economics of Aging, Chicago: University of Chicago Press, pp 301-346.

McDowell BJ, Engberg SJ, Rodriguez E, Engberg R, Sereika S. (1996). Characteristics of urinary incontinence in homebound older adults. J Am Geriatr Soc 44(8):963-8.

McDowell, I. (2006). Measuring Health, A Guide to Rating Scales and Questionnaires, Third Edition. Oxford University Press New York, NY

McGeary DD, Mayer TG, Gatchel RJ, Anagnostis C, Proctor TJ. (2003). Gender-related differences in treatment outcomes for patients with musculoskeletal disorders. Spine J 3(3):197-203.

McGwin G Jr, Melton SM, May AK, Rue LW. (2000). Long-term survival in the elderly after trauma. J Trauma. 49(3):470-6.

Michaels AJ, Michaels CE, Smith JS, Moon CH, Peterson C, Long WB. (2000). Outcome from injury: general health, work status, and satisfaction 12 months after trauma. J Trauma. 48(5):841-8

Mock C, MacKenzie E, Jurkovich G, Burgess A, Cushing B, deLateur B, McAndrew M, Morris J, Swiontkowski M. (2000). Determinants of disability after lower extremity fracture. J Trauma 49(6):1002-11.

Nagin D. (1999). Analyzing developmental trajectories: A semiparametric, group-based approach. Psychol. Meth 4:139-157.

Nijs J, Inghelbrecht E, Daenen L, Hachimi-Idrissi S, Hens L, Willems B, Roussel N, Cras P, Bernheim J. (2011). Long-term functioning following whiplash injury: the role of social support and personality traits. Clin Rheumatol 30(7):927-35.

Nirula R, Nirula G, Gentilello LM. (2009). Inequity of rehabilitation services after traumatic injury. J Trauma 66(1):255-9.

Osberg JS, Unsworth CA. (1997). Trauma-rehabilitation connections: discharge and admission decisions for children. Pediatr Rehabil 1(3):131-46.

Overgaard M, Høyer CB, Christensen EF. (2011). Long-term survival and health-related quality of life 6 to 9 years after trauma. J Trauma 71(2):435-41

Pape TL, Lundgren S, Heinemann AW, Guernon A, Giobbie-Hurder A, Wang J, Roth H, Blahnik M, Williams V. (2006). Establishing a prognosis for functional outcome during coma recovery. Brain Inj 20(7):743-58. 
Pfeifer R1, Lichte P, Zelle BA, Sittaro NA, Zilkens A, Kaneshige JR, Pape HC. (2011). Socio-economic outcome after blunt orthopaedic trauma: Implications on injury prevention. Patient Saf Surg. 5(1):9

Polinder S, van Beeck EF, Essink-Bot ML, Toet H, Looman CW, Mulder S, Meerding WJ. (2007). Functional outcome at 2.5, 5, 9, and 24 months after injury in the Netherlands. J Trauma. 62(1):133-41.

Polinder S, Haagsma JA, Belt E, Lyons RA, Erasmus V, Lund J, van Beeck EF. (2010). A systematic review of studies measuring health-related quality of life of general injury populations. BMC Public Health 23;10:783

Ponsford J, Whelan-Goodinson R, and Bahar-Fuchs, A. (2007). Alcohol and drug use following traumatic brain injury: a prospective study. Brain Injury,

21(13/14):1385-1392

Ponsford J. (2013). Factors contributing to outcome following traumatic brain injury. NeuroRehabilitation 32(4):803-15.

Redmill DA, Mcllwee A, McNicholl B, Templeton C. (2006). Long term outcomes 12 years after major trauma. Injury 37(3):243-6.

Reistetter TA, Graham JE, Deutsch A, Markello SJ, Granger CV, Ottenbacher KJ. (2011). Diabetes comorbidity and age influence rehabilitation outcomes after hip fracture. Diabetes Care 34(6):1375-7.

Rhodes M, Aronson J, Moerkirk G, Petrash E. (1988). Quality of life after the trauma center. J Trauma. 28 (7):931-8.

Rice SA, Allaire J, Elgin K, Farrell W, Conaway M, Blackman JA. (2004). Effect of shortened length of stay on functional and educational outcome after pediatric rehabilitation. Am J Phys Med Rehabil (1):27-32.

Rivara FP, Mackenzie EJ, Jurkovich GJ, Nathens AB, Wang J, Scharfstein DO. (2008). Prevalence of pain in patients 1 year after major trauma. Archives of Surgery, 143 (3):282-287

Rose RA, Parish SL, Yoo J, Grady MD, Powell SE, Hicks-Sangster TK. (2010). Suppression of racial disparities for children with special health care needs among families receiving Medicaid. Soc Sci Med. 70(9):1263-70

Rosen H, Saleh F, Lipsitz S, Rogers SO Jr, Gawande AA. (2009). Downwardly mobile: the accidental cost of being uninsured. Arch Surg 144(11):1006-11

Sabharwal S, Zhao C, McClemens E, Kaufmann A. (2007). Pediatric orthopaedic patients presenting to a university emergency department after visiting another emergency department: demographics and health insurance status. J Pediatr Orthop 27(6):690-4.

Sacks GD, Hill C, Rogers SO Jr. (2011). Insurance status and hospital discharge disposition after trauma: inequities in access to postacute care. $J$ Trauma 71(4):1011-5.

Saxon L, Finc C, and Bass S. (1999). Sports participation, sports injuries, and osteoarthritis: implications for prevention. Sports Medicine 28(2):123-135

Schatz P, Hughes LJ, Chute DL. (2001). Underutilization of neuropsychology in traumatic brain injury rehabilitation: is managed care to blame?

NeuroRehabilitation 16(4):281-7. 
Selassie AW, Pickelsimer EE, Frazier L Jr, Ferguson PL. (2004). The effect of insurance status, race, and gender on ED disposition of persons with traumatic brain injury. Am J Emerg Med 22(6):465-73.

Shafi S, Marquez de la Plata C, Diaz-Arrastia R, Shipman K, Carlile M, Frankel H, Parks J, Gentilello LM. (2007). Racial disparities in long-term functional outcome after traumatic brain injury. $J$ Trauma. 63(6):1263-8

Shafi S, de la Plata CM, Diaz-Arrastia R, Bransky A, Frankel H, Elliott AC, Parks J, Gentilello LM. (2007). Ethnic disparities exist in trauma care. J Trauma 63(5):1138-42.

Singer MB, Liou DZ, Clond MA, Bukur M, Mirocha J, Margulies DR, Salim A, Ley EJ. (2013). Insurance-and race-related disparities decrease in elderly trauma patients. $J$ Trauma 74(1):312-6.

Slomine BS, McCarthy ML, Ding R, MacKenzie EJ, Jaffe KM, Aitken ME, Durbin DR, Christensen JR, Dorsch AM, Paidas CN; CHAT Study Group. (2006). Health care utilization and needs after pediatric traumatic brain injury. Pediatrics 117(4):e663-74.

Sluys K, Häggmark T, Iselius L. (2005). Outcome and quality of life 5 years after major trauma. J Trauma 59(1):223-32.

Soberg HL, Bautz-Holter E, Roise O, Finset A. (2007). Long-term multidimensional functional consequences of severe multiple injuries two years after trauma: a prospective longitudinal cohort study. J Trauma 62(2):461-70.

Soberg HL, Finset A, Roise O, Bautz-Holter E. (2012). The trajectory of physical and mental health from injury to 5 years after multiple trauma: a prospective, longitudinal cohort study. Arch Phys Med Rehabil 93(5):765-74

Sorensen MD, Wessells H, Rivara FP, Zonies DH, Jurkovich GJ, Wang J, Mackenzie EJ. (2008). Prevalence and predictors of sexual dysfunction 12 months after major trauma: a national study. J Trauma. 65(5):1045-52

Staudenmayer KL, Diaz-Arrastia R, de Oliveira A, Gentilello LM, Shafi S. (2007). Ethnic disparities in long-term functional outcomes after traumatic brain injury. $J$ Trauma 63(6):1364-9.

Stevens JA, Corso PS, Finkelstein EA, Miller TR. (2006). The costs of fatal and non-fatal falls among older adults. Injury Prevention. 12:290-295.

Stewart AL, Ware JE Jr, Brook RH. (1981). Advances in the measurement of functional status: construction of aggregate indexes. Med Care 19(5):473-88.

Stewart AL, Greenfield S, Hays RD, Wells K, Rogers WH, Berry SD, McGlynn EA, Ware JE Jr. (1989). Functional status and well-being of patients with chronic conditions. Results from the Medical Outcomes Study. JAMA 18;262(7):907-13.

Suurmeijer T, Reuvekamp M, Aldenkamp B. (2002). Social Functioning, Psychological Functioning, and Quality of Life in Epilepsy. Epilepsia 42(9):1160-1168

Taghavi S, Jayarajan SN, Duran JM, Gaughan JP, Pathak A, Santora TA, Willis AI, Goldberg AJ. (2012). Does payer status matter in predicting penetrating trauma outcomes? Surgery 152(2):227-31.

Tate DG, Stiers W, Daugherty J, Forchheimer M, Cohen E, Hansen N. (1994). The effects of insurance benefits coverage on functional and psychosocial outcomes after spinal cord injury. Arch Phys Med Rehabil 75(4):407-14. 
Tate DG, Forchheimer M, Daugherty J, Maynard F. (1994). Determining differences in post discharge outcomes among catastrophically and noncatastrophically sponsored outpatients with spinal cord injury. Am J Phys Med Rehabil 73(2):8997.

Timmers TK, Verhofstad MH, Moons KG, van Beeck EF, Leenen LP. (2011). Long-term quality of life after surgical intensive care admission. Arch Surg 146(4):412-8

Tomey KM, Sowers MR. (2009). Assessment of physical functioning: a conceptual model encompassing environmental factors and individual compensation strategies. Phys Ther 89(7):705-14.

Tsaousides T, Warshowsky A, Ashman TA, Cantor JB, Spielman L, Gordon WA. (2009). The relationship between employment-related self-efficacy and quality of life following traumatic brain injury. Rehabil Psychol. 54(3):299-305.

Ulvik A, Kvåle R, Wentzel-Larsen T, Flaatten H. (2008). Quality of life 2-7 years after major trauma. Acta Anaesthesiol Scand. 52(2):195-201.

Watson WL, Ozanne-Smith J, Richardson J. (2007). Retrospective baseline measurement of self-reported health status and health-related quality of life versus population norms in the evaluation of post-injury losses. Inj Prev. 13(1):45-50.

Wegener ST, Castillo RC, Haythornthwaite J, Mackenzie EJ, Bosse MJ; LEAP Study Group. (2011). Psychological distress mediates the effect of pain on function. Pain 152(6):1349-57.

White FA, French D, Zwemer FL Jr, Fairbanks RJ. (2007). Care without coverage: is there a relationship between insurance and ED care? J Emerg Med 32(2):159-65.

Whitnall L, McMillan TM, Murray GD, Teasdale GM. (2006). Disability in young people and adults after head injury: 5-7 year follow up of a prospective cohort study. J Neurol Neurosurg Psychiatry 77(5):640-5.

Wilson IB, Cleary PD. (1995). Linking clinical variables with health-related quality of life. A conceptual model of patient outcomes. JAMA 4;273(1):59-65.

Woon FL, Dunn CB, Hopkins RO. (2012). Predicting cognitive sequelae in survivors of critical illness with cognitive screening tests. Am J Respir Crit Care Med 15;186(4):333-40

World Health Organization. (1980). The International Classification of Impairments, Disabilities and Handicaps. Available at http://whqlibdoc.who.int/publications/1980/9241541261 eng.pdf

World Health Organization. (1996). Measuring Quality of Life. Available at http://www.who.int/mental_health/media/68.pdf

World Health Organization. (2001). The International Classification of Functioning, Disability and Health. Available at http://www.who.int/classifications/docs/en/

Wyman JF, Henly SJ. (2011). Advancing nursing science through health trajectory research: an introduction. Nurs Res 60(3 Suppl):S1-4.

Zatzick DF, Rivara FP, Nathens AB, Jurkovich GJ, Wang J, Fan MY, Russo J, Salkever DS, Mackenzie EJ. (2007). A nationwide US study of post-traumatic stress after hospitalization for physical injury. Psychol Med 37(10):1469-80.

Zatzick D, Jurkovich GJ, Rivara FP, Wang J, Fan MY, Joesch J, Mackenzie E. (2008). A national US study of posttraumatic stress disorder, depression, and work and functional outcomes after hospitalization for traumatic injury. Ann Surg 248(3):429-37. 
Zwerling C, Sprince NL, Wallace RB, Davis CS, Whitten PS, Heeringa SG. (1995). Effect of recall period on the reporting of occupational injuries among older workers in the Health and Retirement Study. Am J Ind Med 28:583-590 


\section{VITA}

Teresa Maria Bell was born in St. Louis, MO in 1984. She completed her Bachelor of Science degree in neuroscience from Rhodes College in Memphis, TN in 2007. She then went on to work at St. Jude Children's Research Hospital in the Division of Bone Marrow Transplant and Cellular Therapy from 2007-2010. In fall of 2010, Teresa began work on her doctorate in Health Outcomes and Policy Research at the University of Tennessee Health Science Center. Teresa served as the Graduate School Executive Council president from 2011-2012 and as the Interprofessional Student Council president from 2013-2014. She also completed a graduate certificate in Geographic Information Science from the University of Memphis in 2012. While completing her Doctor of Philosophy degree, Teresa worked as a graduate research assistant in the Department of Surgery. Teresa will receive the Doctor of Philosophy degree from the University of Tennessee Health Science Center in May 2014. 